\title{
Lactol-directed Osmylation. Stereodivergent synthesis of four C-19,20 Apoptolidin diols from a single allylic hemiacetal
}

\author{
Youngsoon Kim and Philip L. Fuchs* \\ Department of Chemistry, Purdue University, West Lafayette, Indiana 47907 \\ pfuchs@purdue.edu
}

\section{Supporting Information}

\section{GENERAL PROCEDURES}

All reagents purchased were used as received. Tetrahydrofuran (THF) and diethyl ether were distilled from benzophenone ketyl. Benzene, toluene, and methylene chloride $\left(\mathrm{CH}_{2} \mathrm{Cl}_{2}\right)$ were distilled from calcium hydride. Sodium sulfate $\left(\mathrm{Na}_{2} \mathrm{SO}_{4}\right)$ was anhydrous. All recrystalization, chromatographic, and workup solvents were distilled. Unless otherwise indicated, all reactions were carried out under in a positive pressure of nitrogen in anhydrous solvents and the reaction flasks were fitted with rubber septa for the introduction of substrates and reagents via syringe. Progress of reactions was monitored by thin layer chromatography (TLC) using silica gel 60 F-254 plates (EM reagents, 0.25 $\mathrm{mm})$. The TLC plates were visualized with a UV lamp (254 nm) and/or with TLC visualizing solutions activated with heat. The two commonly employed TLC visualizing solutions were: (i) p-anisaldehyde solution $(1350 \mathrm{~mL}$ absolute ethanol, $50 \mathrm{~mL}$ 
concentrated $\mathrm{H}_{2} \mathrm{SO}_{4}, 37 \mathrm{~mL}$-anisaldehyde), and (ii) permanganate solution (weight percents of $1 \% \mathrm{KMnO}_{4}$ and $2 \% \mathrm{Na}_{2} \mathrm{CO}_{3}$ in water).

${ }^{1} \mathrm{H}$ NMR and ${ }^{13} \mathrm{C}$ NMR spectra were recorded on $300 \mathrm{MHz}, 400 \mathrm{MHz}$ or 500 MHz. Because of a lability of the allylic hemiacetal and acetals, the NMR spectra were determined in benzene- $\mathrm{d}_{6}\left(\mathrm{C}_{6} \mathrm{D}_{6}\right)$ solution and are reported in parts per million (ppm) from the residual benzene $(7.15 \mathrm{ppm}$ and $128.00 \mathrm{ppm})$ standard respectively. Peak multiplicities in ${ }^{1} \mathrm{H}-\mathrm{NMR}$ spectra, when reported, are abbreviated as s (singlet), d (doublet), t (triplet), m (multiplet), dd (doublet of doublet), td (triplet of doublet) and/or quint (quintet). Melting points were obtained on a capillary melting point apparatus or automated melting point system and are uncorrected. Mass spectra were run by the Purdue University campus wide mass spectrometry facility. Because of a stability of the hemiacetal and acetals, the Mass spectra were determined by ESI technique. 


\section{Preparation of $\mathbf{2}$}
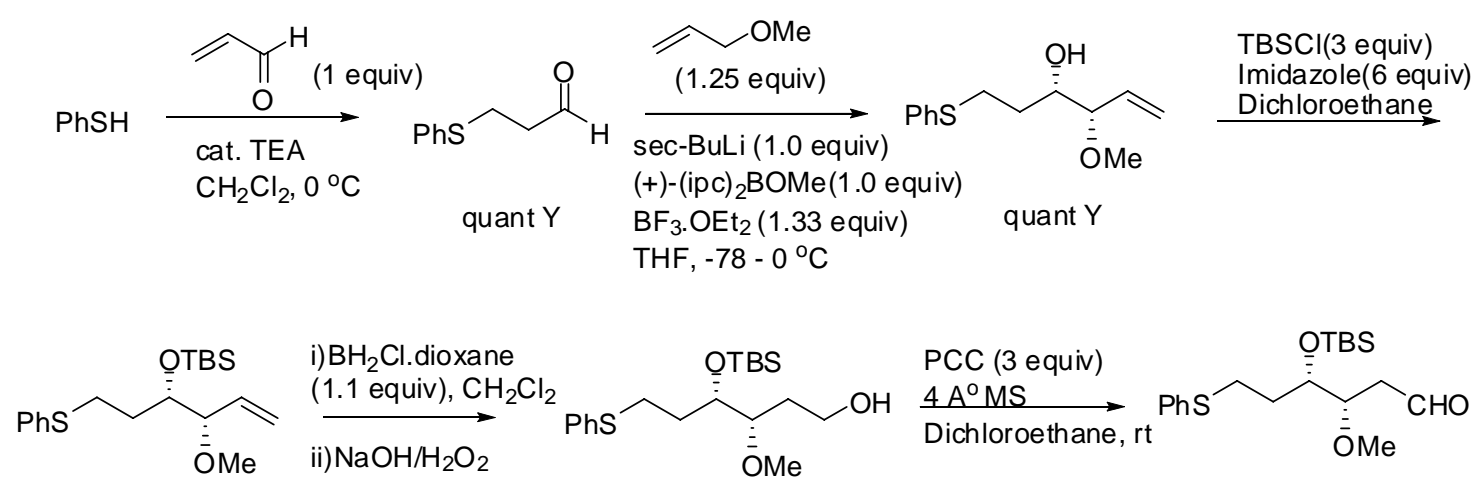

$80 \%$

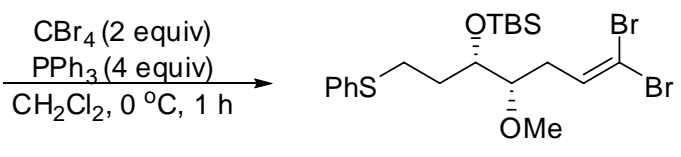

$70 \%$

2

\section{Preparation of 3}

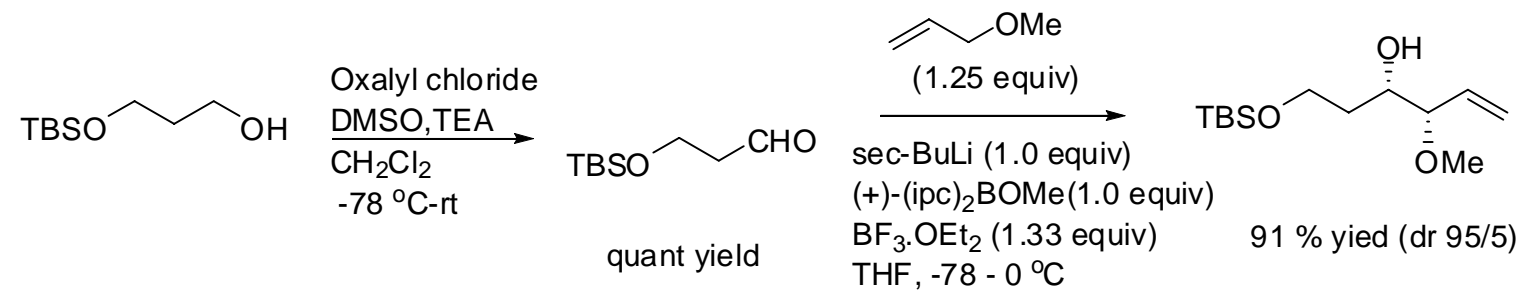

TBSOTf

2,6-lutidine

$\stackrel{\mathrm{CH}_{2} \mathrm{Cl}_{2},-78^{\circ} \mathrm{C}}{\longrightarrow}$

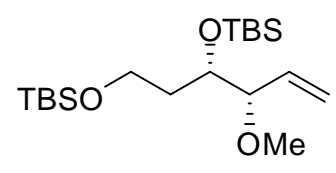

i) $\mathrm{BH}_{2} \mathrm{Cl} . \mathrm{SMe}_{2}$

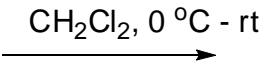

ii) $\mathrm{NaOH} / 50 \% \mathrm{H}_{2} \mathrm{O}_{2}$

quant $Y$

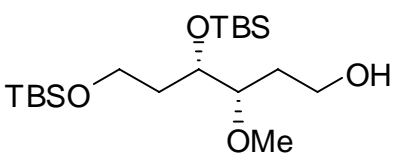

$80-85 \%$

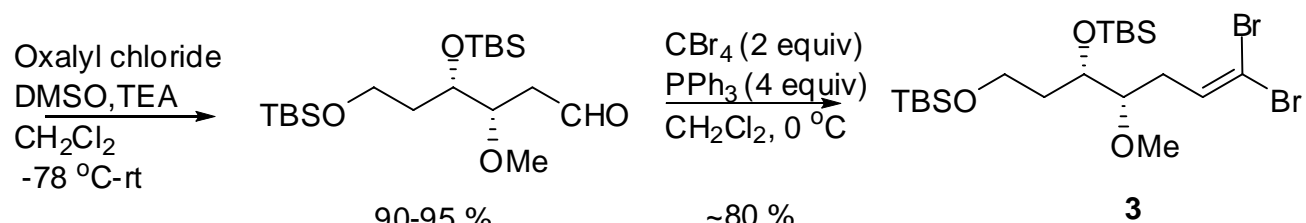

Improved preparation of lactone $\mathbf{4}$ 


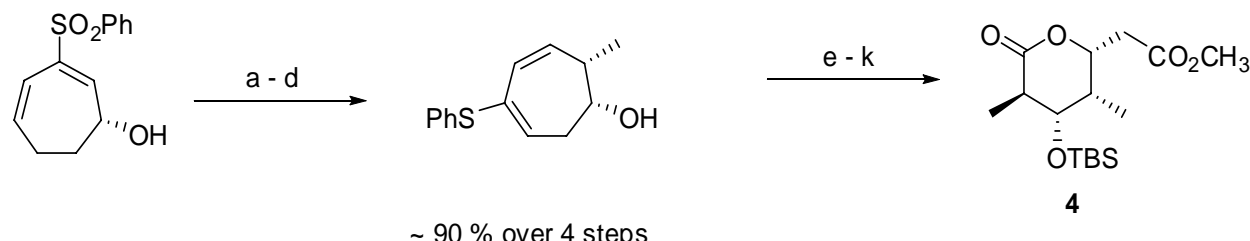

diastereoselectivity $\sim 13: 1 \quad 35 \sim 42 \%$ over 7 steps

(a) Hexamethyldisilazane (1.1 eq) / TMSCl (0.1 eq), $\mathrm{CH}_{2} \mathrm{Cl}_{2}(0.5 \mathrm{M}), 0{ }^{\circ} \mathrm{C}-\mathrm{rt} * \quad$ (b) i) $\mathrm{CH}_{3} \mathrm{Li}(1.3 \mathrm{eq}), \mathrm{THF},-78{ }^{\circ} \mathrm{C}$ ii) $\mathrm{PhSSPh}(1.5$

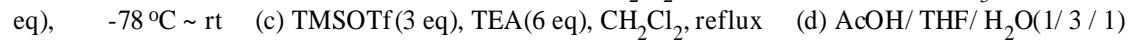

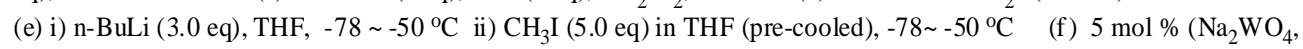

$\mathrm{Oct}_{3} \mathrm{MeNHSO}_{4}, \mathrm{PhPO}(\mathrm{OH})_{2}$ ), $30 \% \mathrm{H}_{2} \mathrm{O}_{2}$ (3 eq), EtOAc, rt, $10 \mathrm{~h}$ (g) TBSOTf, TEA, MC, $-78 \sim 0{ }^{\circ} \mathrm{C}$ (h) Oxone (5.5 eq),

$\mathrm{NaHCO}_{3}(19 \mathrm{eq})$, Acetone- $\mathrm{H}_{2} \mathrm{O}(3: 2), 20 \mathrm{~h}$, rt $\quad$ (i) DIBAL-H (1.5 eq), THF (1.0 eq), Toluene, $-78 \sim-60{ }^{\circ} \mathrm{C}, 6 \mathrm{~h} \quad$ (j) i) $\mathrm{O}_{3}$,

$\mathrm{NaHCO}_{3}(4.0 \mathrm{eq}), \mathrm{MeOH}-\mathrm{CH}_{2} \mathrm{Cl}_{2}(2: 1),-78{ }^{\circ} \mathrm{C}, 30 \mathrm{~min}$ ii) $\mathrm{Me}_{2} \mathrm{~S}$ quench $\quad$ (k) $5 \mathrm{~mol} \% \mathrm{RuCl}_{3} \cdot 3 \mathrm{H}_{2} \mathrm{O}, \mathrm{NaBrO}_{3}(3 \mathrm{eq}), \mathrm{CH}_{3} \mathrm{CN}-$

EtOAc-pH 7 buffer (1:1:2), $0{ }^{\circ} \mathrm{C} \sim \mathrm{rt}, 3 \mathrm{~h}$

* Because of lability of TMS, quick celite filter only. (avoid silicagel filter and aqueous work-up.)

$\underline{\text { Detailed procedure for step (e) }}$

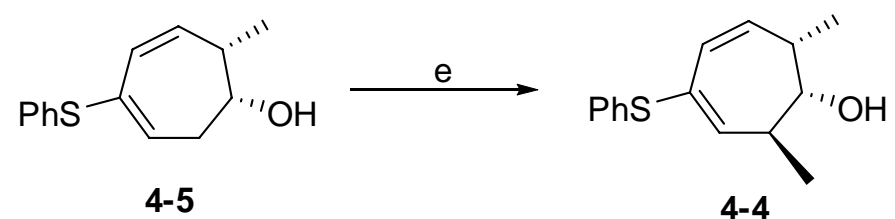

A solution of alcohol 4-5 (4.75 g, $20.4 \mathrm{mmol})$ in THF (204 ml) was cooled to $-78{ }^{\circ} \mathrm{C}$, and $24.5 \mathrm{ml}$ of $\mathrm{n}-\mathrm{BuLi}$ (2.5 $\mathrm{M}$ in hexane, $61.3 \mathrm{mmol}$ ) was added dropwise. The mixture was slowly brought to $-50{ }^{\circ} \mathrm{C}$ over $1.5 \mathrm{~h}$, keep stirring at $-50{ }^{\circ} \mathrm{C}$ for $1 \mathrm{~h}$, and then cooled back to $-78{ }^{\circ} \mathrm{C}$. To this cold dark orange solution was slowly added a pre-cooled solution (at $-78{ }^{\circ} \mathrm{C}$ ) of $\mathrm{MeI}(6.4 \mathrm{ml}, 102.2 \mathrm{mmol})$ in THF $(50 \mathrm{ml})$ over $20 \mathrm{~min}$. The resulting yellow solution was warmed to $-50{ }^{\circ} \mathrm{C}$ over $3 \mathrm{~h}$. The reaction was quenched with $\mathrm{MeOH}$ (10 ml), followed by aq $\mathrm{Na}_{2} \mathrm{SO}_{4}$ solution ${ }^{1}(50 \mathrm{ml})$. After warming to $0{ }^{\circ} \mathrm{C}$, the crude mixture was transferred to a separatory funnel and extracted with ethyl acetate $(3 \times 100$ $\mathrm{mL}$ ), dried with $\mathrm{Na}_{2} \mathrm{SO}_{4}$ and concentrated using a rotary evaporator to give a crude antimethylated product 4-4 (5.0 g, purity 80 85 \%) as a yellow solid. ${ }^{2}$ The crude material is used in the next step.

\footnotetext{
${ }^{1}$ Avoid brine (chloride source) work-up for next Noyori oxidation.

${ }^{2}$ The pure product is a white solid. (m.p ; $81.5-83.5^{\circ} \mathrm{C}$ )
} 


\section{Detailed procedure for step (h)}

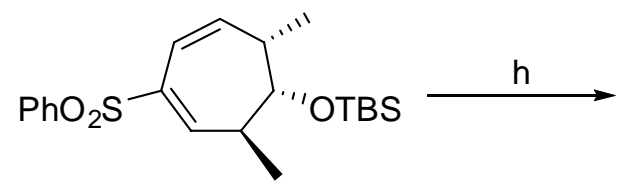

4-3

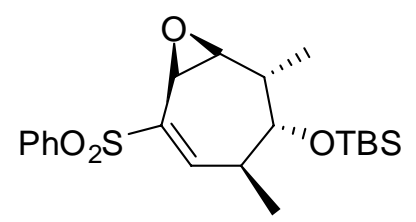

4-2

To a $1 \mathrm{~L}$ 3-neck round bottom flask fitted with a mechanical stirrer was added dienyl sulfone 4-3 (9.70 g, $24.7 \mathrm{mmol})$, acetone $(124 \mathrm{~mL})$ and water $(82 \mathrm{ml})$ at room temperature. After cooling to $0 \sim 5{ }^{\circ} \mathrm{C}, \mathrm{NaHCO}_{3}(18.7 \mathrm{~g}, 222 \mathrm{mmol})$ was added to the above mixture to give a fine white suspension. OXONE ${ }^{\circledR}$ (38.0 g, $\left.61.7 \mathrm{mmol}\right)$ was added in 3 portions at $10 \mathrm{~min}$ intervals at $0 \sim 5{ }^{\circ} \mathrm{C}$ with vigorous stirring. After $4 \sim 5 \mathrm{~h}$ vigorous stirring at rt, an additional $\mathrm{NaHCO}_{3}(18.7 \mathrm{~g}, 222 \mathrm{mmol})$ was added, followed by $\mathrm{OXONE}^{\circledR}(38.0 \mathrm{~g}, 61.7 \mathrm{mmol})$ at $0 \sim 5{ }^{\circ} \mathrm{C}$. After $4 \sim 5 \mathrm{~h}$ additional stirring at $\mathrm{rt}$, the reaction was judged complete by TLC and the mixture was transferred to a separatory funnel. Water $(700 \mathrm{ml})$ was added and extracted with ethyl acetate $(2 \times 700 \mathrm{~mL})$. The combined organic layers were washed with brine, dried over $\mathrm{Na}_{2} \mathrm{SO}_{4}$, concentrated using a rotary evaporator to give a $\beta$-epoxide 4-2 (10.0 g, purity 90 \%) as a yellow oil.

Detailed procedure for step $(\mathrm{k})$

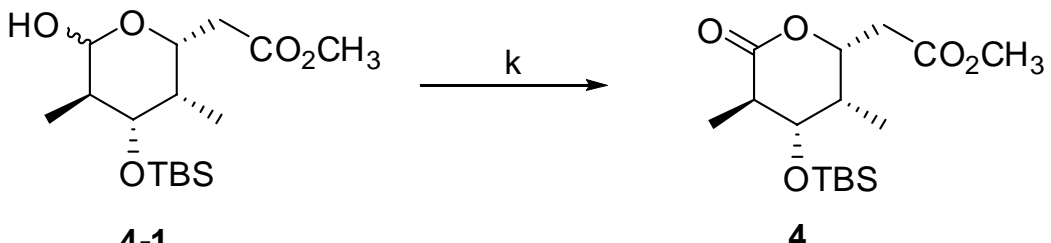

4-1

To a solution of lactol 4-1 (1.11 g, $3.34 \mathrm{mmol})$, phosphate buffer (16 ml, $\mathrm{pH} 7.48)$, ethyl acetate $(8 \mathrm{ml})$ and acetonitrile $(8 \mathrm{ml})$ was added $\mathrm{NaBrO}_{3}(1.51 \mathrm{~g}, 10.01 \mathrm{mmol})$ at 0 ${ }^{\circ} \mathrm{C}$. After adding $5 \mathrm{~mol} \% \mathrm{RuCl}_{3} \bullet 3 \mathrm{H}_{2} \mathrm{O}(0.04 \mathrm{~g}, 0.17 \mathrm{mmol})$, the mixture was stirred for 30 minuets at $0{ }^{\circ} \mathrm{C}$. After additional stirring around $6 \mathrm{~h}$ at rt, reaction monitored by TLC. 
The reaction was quenched with sat. $\mathrm{Na}_{2} \mathrm{~S}_{2} \mathrm{O}_{3}$ at $0{ }^{\circ} \mathrm{C}$, passed through celite (3 inch) to remove the black precipitate, and then washed with ethyl acetate $(20 \mathrm{ml})$. After the organic phase was separated, the aqueous phase was extracted with ethyl acetate (30 $\mathrm{ml} \times$ 3), dried with $\mathrm{Na}_{2} \mathrm{SO}_{4}$ and concentrated using a rotary evaporator to give a yellow oil. The crude oil was purified by flash column chromatography (hexanes: ethyl acetate $=$ 7:3) to afford $0.99 \mathrm{~g}(90 \%)$ of lactone 4 as an oil.

\section{Preparation of hemiacetal $\mathbf{5}$}

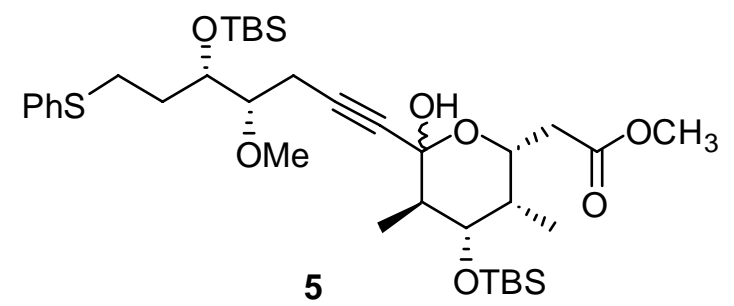

To a stirred solution of $2(671 \mathrm{mg}, 1.28 \mathrm{mmol})$ in THF $(12.8 \mathrm{~mL})$ at $-78{ }^{\circ} \mathrm{C}$ was slowly added n-butyllithium (1.02 mL, $2.56 \mathrm{mmol}, 2.5 \mathrm{M}$ solution in hexane). After $1 \mathrm{~h}$ stirring at $-78{ }^{\circ} \mathrm{C}$, the reaction mixture was slowly added to lactone 4 (423mg, $1.28 \mathrm{mmol}$ ) in THF (6.4ml). The temperature was allowed to increase to $-50{ }^{\circ} \mathrm{C}$ and the reaction maintained for $5 \mathrm{~h}$ at $-50{ }^{\circ} \mathrm{C}$. The reaction was quenched with $\mathrm{MeOH}$ followed by sat. $\mathrm{NH}_{4} \mathrm{Cl}$. The crude mixture was transferred to a separatory funnel and extracted with ethyl acetate $(2 \times 15 \mathrm{~mL})$, dried with $\mathrm{Na}_{2} \mathrm{SO}_{4}$ and concentrated using a rotary evaporator to give a light yellow oil. The crude oil was purified by flash column chromatography (hexanes: ethyl acetate $=4: 1)$ to afford $667 \mathrm{mg}(75 \%)$ of an $\alpha, \beta$-anomeric mixture $(\sim 1: 1)$ of 5 as an oil, along with recovered lactone 4 ( $20 \%$ ) and the acetylene derived from 2 ( 20\%).

$\alpha, \beta-5 ;{ }^{1} \mathrm{H}$ NMR $\left(\mathrm{C}_{6} \mathrm{D}_{6}, 400 \mathrm{MHz}\right) \delta 7.37$ (m, 4H), 7.07 (m, 3H), 6.94 (m, 3H), 4.77 (m, 1H), 4.68(m, 1H), 4.12 (quint, $J=4.4 \mathrm{~Hz} 1 \mathrm{H}$ ), 4.04 (m, 3H), 3.90 (dd, $J=4.7 \mathrm{~Hz}$, 10.7Hz, 1H), 3.37 (s, 3H), 3.34 (s, 3H), 3.30 (s, 3H), 3.13 (s, 3H), 3.08 (m, 1H), 2.97 (m, 
4H), 2.72 (dd, $J=8.7 \mathrm{~Hz}, 15.7 \mathrm{~Hz}, 1 \mathrm{H}), 2.66$ (dd, $J=9.2 \mathrm{~Hz}, 16.1 \mathrm{~Hz}, 1 \mathrm{H}), 2.55$ (dd, $J=$ $3.9 \mathrm{~Hz}, 17.1 \mathrm{~Hz}, 1 \mathrm{H}), 2.43$ (dd, $J=4.6 \mathrm{~Hz}, 17.1 \mathrm{~Hz}, 1 \mathrm{H}), 2.23$ (m, 6H), 2.05 (m, 3H), 1.88 (m, 2H), 1.72 (m, 1H), 1.45 (d, $J=6.7 \mathrm{~Hz}, 3 \mathrm{H}), 1.36$ (d, $J=6.5 \mathrm{~Hz}, 3 \mathrm{H}), 1.01$ (d, $J=6.9$ Hz, 3H), 0.98 (d, $J=6.9 \mathrm{~Hz}, 3 \mathrm{H}), 0.97(\mathrm{~s}, 12 \mathrm{H}), 0.94(\mathrm{~s}, 12 \mathrm{H}), 0.92(\mathrm{~s}, 12 \mathrm{H}), 0.16(\mathrm{~s}, 3 \mathrm{H})$, 0.13(s, 3H), 0.10 (s, 3H), 0.08 (s, 3H), 0.07 (s, 3H), 0.06 (s, 3H), 0.04 (s, 3H), 0.00 (s, $3 \mathrm{H}) ;{ }^{13} \mathrm{C}$ NMR $\left(\mathrm{C}_{6} \mathrm{D}_{6}, 400 \mathrm{MHz}\right) \quad \delta 171.5,171.2,137.2,129.3,129.2,129.1,128.2$, 127.7, 125.9, 96.8, 95.1, 85.1, 82.9, 82.6, 82.3, 80.4, 78.8, 75.1, 72.7, 71.5, 71.4, 70.9, 68.1, 57.9, 57.8, 51.1, 42.6, 41.7, 39.3, 39.0, 37.7, 37.6, 31.8, 31.5, 30.0, 29.9, 26.0, 25.9, 19.2, 19.1, 18.2, 18.2, 13.7, 13.2, 5.8, 5.3, -4.1, -4.2, -4.3, -4.5, -4.6, -4.7. HRMS (ESI) calculated for $\mathrm{C}_{36} \mathrm{H}_{62} \mathrm{O}_{7} \mathrm{SSi}_{2}(\mathrm{M}+\mathrm{Na})$ 717.3653, found 717.3659.

\section{$\underline{\text { Preparation of hemiacetal } 6}$}

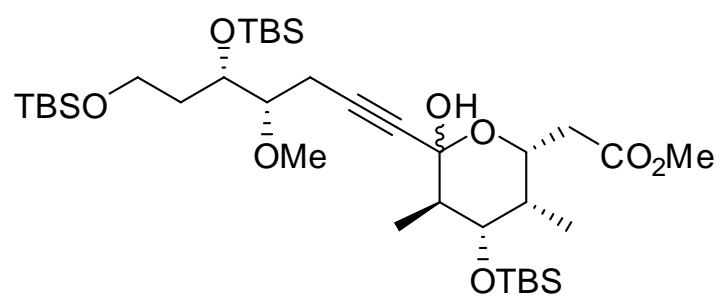

The procedure is same as used with hemiacetal 5 . The product 6 obtained an $\alpha, \beta$ anomeric mixture $(\sim 1: 1)$ as an oil in 80\% yield, along with recovered lactone 4 ( 20\%) and the acetylene from 3 ( 20\%).

$\alpha, \beta-6 ;{ }^{1} \mathrm{H}$ NMR $\left(\mathrm{C}_{6} \mathrm{D}_{6}, 400 \mathrm{MHz}\right) \delta 4.77$ (m, 1H), $4.69(\mathrm{~m}, 1 \mathrm{H}), 4.17(\mathrm{~m}, 1 \mathrm{H}), 4.12$ (quint, $J=4.4 \mathrm{~Hz}, 1 \mathrm{H}), 4.02$ (dd, $J=4.7 \mathrm{~Hz}, 10.6 \mathrm{~Hz}, 1 \mathrm{H}), 3.91(\mathrm{~m}, 2 \mathrm{H}), 3.79(\mathrm{~m}, 4 \mathrm{H}), 3.51$ (m,1H), 3.40 (s, 3H), 3.39 (s, 3H), 3.35 (s, 3H), 3.23 (s, 3H), 2.73 (m, 3H), 2.55 (dd, J = 4.3 Hz, 16.9Hz, 1H), 2.40 (m, 5H), 2.08 (m, 1H), 1.98 (m, 3H), 1.83 (m, 1H), 1.61 (m, 2H), 1.45 (d, $J=6.7 \mathrm{~Hz}, 3 \mathrm{H}), 1.37$ (d, $J=6.6 \mathrm{~Hz}, 3 \mathrm{H}), 1.01(\mathrm{~s}, 9 \mathrm{H}), 0.98$ (s, 9H), 0.97 (s, 9H), 0.96 (s, 18H), 0.94 (s, 9H), 0.19 (s, 3H), 0.16 (s, 3H), 0.12 (s, 6H), 0.08 (s, 6H), 0.07 (s, 12H), 0.04 (s, 3H), 0.00 (s, 3H); ${ }^{13} \mathrm{C}$ NMR $\left(\mathrm{C}_{6} \mathrm{D}_{6}, 400 \mathrm{MHz}\right) \delta 171.6,171.2,96.9$, 95.1, 85.4, 83.2, 82.7, 82.5, 80.6, 78.8, 75.1, 72.8, 70.8, 69.7, 69.5, 67.9, 59.6, 59.5, 
57.99, 57.92, 51.1, 42.6, 41.7, 39.3, 39.1, 37.7, 37.6, 35.4, 34.9, 26.1, 26.0, 19.4, 18.4, 18.3, 18.2, 13.7, 13.2, 5.8, 5.3, -4.1, -4.2, -4.6, -4.7, -5.1. HRMS (ESI) calculated for $\mathrm{C}_{36} \mathrm{H}_{72} \mathrm{O}_{8} \mathrm{Si}_{3}(\mathrm{M}+\mathrm{Na})$ 739.4433, found 739.4438.

Preparation of hemiacetal 7

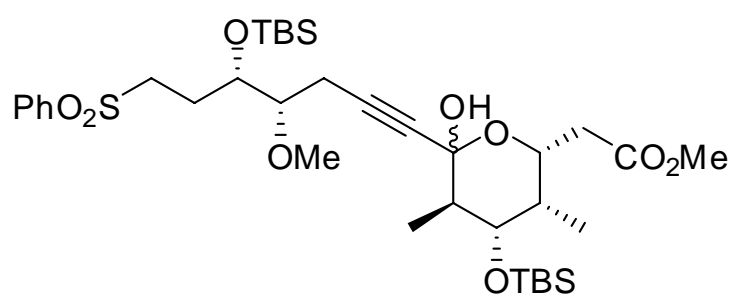

To a stirred solution of 5 (660 $\mathrm{mg}, 0.95 \mathrm{mmol})$ in ethyl acetate $(4.7 \mathrm{~mL})$ were added 5 mol \% $1 \mathrm{M}$ aqueous $\mathrm{Na}_{2} \mathrm{WO}_{4} \cdot 2 \mathrm{H}_{2} \mathrm{O}(47.4 \mu \mathrm{l}), 5 \mathrm{~mol} \% 1 \mathrm{M}$ aqueous $\mathrm{PhPO}(\mathrm{OH})_{2}(47.4$ $\mu \mathrm{l}$ ), $5 \mathrm{~mol} \% 0.5 \mathrm{M} \mathrm{Oct}_{3} \mathrm{MeNHSO}_{4}$ (in Toluene, $94.8 \mu \mathrm{l}$ ) and $30 \mathrm{wt} \% \mathrm{H}_{2} \mathrm{O}_{2}(0.29 \mathrm{ml}$, $2.85 \mathrm{mmol}$ ) at $0{ }^{\circ} \mathrm{C}$. The ice bath was immediately removed and the reaction allowed to warm to rt. After $10 \mathrm{~h}$ stirring at rt, the reaction was quenched with sat. $\mathrm{Na}_{2} \mathrm{~S}_{2} \mathrm{O}_{3}$. The crude mixture was transferred to a separatory funnel and extracted with ethyl acetate $(2 \times$ $15 \mathrm{~mL}$ ), washed with brine, dried with $\mathrm{Na}_{2} \mathrm{SO}_{4}$ and concentrated using a rotary evaporator to give a light yellow oil. The crude oil was purified by flash column chromatography (hexanes: ethyl acetate $=7: 3$ ) to afford $640 \mathrm{mg}(93 \%)$ of $\alpha, \beta$-anomeric mixture ( 1: 1) 7 as a foam.

$\alpha, \beta-7$; ${ }^{1} \mathrm{H}$ NMR $\left(\mathrm{C}_{6} \mathrm{D}_{6}, 400 \mathrm{MHz}\right) \delta 7.98$ (m, 2H), 7.90 (m, 2H), 7.04 (m, 6H), 4.91 (s, 1H), 4.84 (m, 1H), 4.07(dd, $J=4.9 \mathrm{~Hz}, 9.7 \mathrm{~Hz}, 1 \mathrm{H}), 3.96$ (d, $J=1.2 \mathrm{~Hz}, 1 \mathrm{H}), 3.90$ (m, 3H), 3.39 (s, 3H), 3.24 (s, 3H), 3.13 (s, 3H), 3.03 (s, 3H), 2.96 (m, 1H), 2.72 (quint, $J=$ 8.6 Hz, 2H), 2.30 (m, 11H), 1.88 (m, 2H), 1.44 (d, $J=6.7 \mathrm{~Hz}, 3 \mathrm{H}), 1.42$ (d, $J=6.6 \mathrm{~Hz}$, 3H), 1.03 (d, $J=5.2 \mathrm{~Hz}, 3 \mathrm{H}), 1.01$ (d, $J=5.4 \mathrm{~Hz}, 3 \mathrm{H}), 0.96$ (s, 9H), 0.94 (s, 9H), 0.88 (s, 9H), 0.83 (s, 9H), 0.12 (s, 3H), 0.08 (s, 3H), 0.05 (s, 6H), 0.03 (s, 3H), 0.02 (s, 3H), 0.00 (s, 3H), -0.02 (s, 3H); ${ }^{13} \mathrm{C}$ NMR $\left(\mathrm{C}_{6} \mathrm{D}_{6}, 400 \mathrm{MHz}\right) \delta 171.5,140.2,140.0133 .2,129.2$, 129.1, 128.4, 128.3, 127.9, 96.8, 95.0, 83.5, 83.2, 82.4, 82.0, 79.9, 79.4, 75.1, 72.8, 72.6, 
71.6, 70.6, 68.1, 57.6, 52.5, 52.2, 51.3, 51.1, 42.5, 41.7, 39.2, 37.7, 26.0, 19.7, 19.2, 18.29, 18.25, 18.1, 13.7, 13.3, 6.0, 5.3, -4.1, -4.2, -4.3, -4.4, -4.6, -4.7, -4.8. HRMS (ESI) calculated for $\mathrm{C}_{36} \mathrm{H}_{62} \mathrm{O}_{9} \mathrm{SSi}_{2}(\mathrm{M}+\mathrm{Na})$ 749.3551, found 749.3558.

\section{$\underline{\text { Preparation of Z-allylic hemiacetal } 8}$}

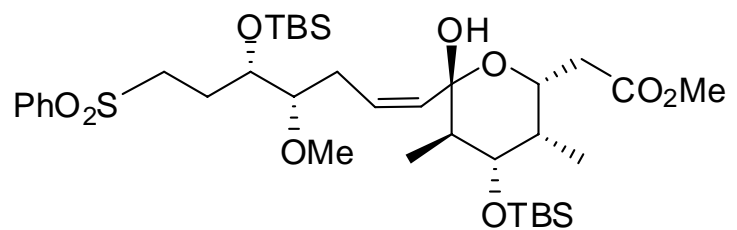

To a stirred solution of 7 (300 $\mathrm{mg}, 0.41 \mathrm{mmol})$ in benzene $(10.3 \mathrm{~mL})$ were added quinoline ( $97.5 \mu \mathrm{l}, 0.82 \mathrm{mmol}$ ) and $10 \mathrm{wt} \%$ palladium on barium sulfate $(30 \mathrm{mg})$. The resulting solution was stirred under Hydrogen balloon. After $8 \mathrm{~h}$ stirring at rt, an additional 2.5 wt \% palladium on barium sulfate $(7.5 \mathrm{mg})$ and benzene $(5 \mathrm{ml})$ were added, and stirred an additional $2 \mathrm{~h}$ at rt. The mixture was then filtered through Celite to remove the catalyst, and the filtrate was concentrated to give a yellow oil. The crude oil was purified by flash column chromatography (hexanes: ethyl acetate $=4: 1$ ) to afford 255 mg (85 \%) of Z-allylic hemiacetal 8 as a single $\beta$-anomer.

8 ; ${ }^{1} \mathrm{H}$ NMR ( $\left.{ }_{6} \mathrm{D}_{6}, 400 \mathrm{MHz}\right) \delta 7.86$ (m, 2H), 6.97 (3s, 3H), 5.60 (d, $\left.J=11.8 \mathrm{~Hz}, 1 \mathrm{H}\right)$, 5.33 (d, $J=1.0 \mathrm{~Hz}, 1 \mathrm{H}), 5.32$ (m, 1H), 4.91 (m, 1H), 4.12 (dd, $J=4.8 \mathrm{~Hz}, 10.4 \mathrm{~Hz}, 1 \mathrm{H}$ ), 3.93 (m, 1H), 3.34 (s, 3H), 3.27 (s, 3H), 3.14 (m,1H), 3.01 (m, 1H), 2.84 (m, 2H), 2.66 (dd, $J=9.6 \mathrm{~Hz}, 15.5 \mathrm{~Hz}, 1 \mathrm{H}$ ), 2.15 (dd, $J=3.9 \mathrm{~Hz}, 15.5 \mathrm{~Hz}, 1 \mathrm{H}$ ), 2.02 (m, 1H), 1.94 (dd, $J=7.9 \mathrm{~Hz}, 12 \mathrm{~Hz}, 1 \mathrm{H}), 1.85$ (m, 2H), 1.71 (m, 1H), 1.20 (d, $J=6.7 \mathrm{~Hz}, 3 \mathrm{H}), 1.03$ (d, $J=$ $6.9 \mathrm{~Hz}, 3 \mathrm{H}), 0.99$ (s, 9H), 0.86 (s, 9H), 0.09 (s, 3H), 0.04 (s, 3H), 0.01 (s, 3H), -0.01 (s, $3 \mathrm{H}) ;{ }^{13} \mathrm{C}$ NMR $\left(\mathrm{C}_{6} \mathrm{D}_{6}, 300 \mathrm{MHz}\right) \delta 171.6,140.2,136.3,133.1,129.1,126.2,100.1$, 83.5, 73.7, 70.3, 66.7, 59.8, 52.3, 50.9, 39.7, 39.6, 38.1, 27.4, 26.0, 25.9, 18.3, 18.0, 12.7, 5.4, -4.1, -4.6, -4.7; HRMS (ESI) calculated for $\mathrm{C}_{36} \mathrm{H}_{64} \mathrm{O}_{9} \mathrm{SSi}_{2}(\mathrm{M}+\mathrm{Na}) 751.3707$, found 751.3712 . 
Preparation of Z-allylic hemiacetal 9

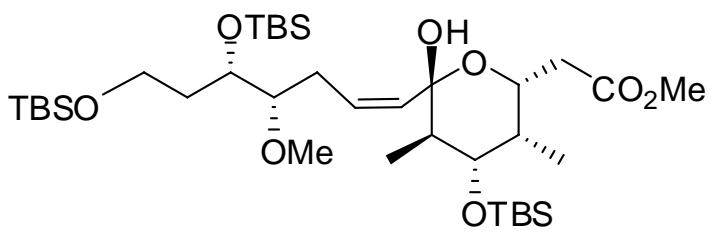

To a stirred solution of $6(420 \mathrm{mg}, 0.58 \mathrm{mmol})$ in benzene $(14.6 \mathrm{~mL})$ were added quinoline (0.14 ml, $1.16 \mathrm{mmol}$ ) and $10 \mathrm{wt} \%$ palladium on barium sulfate (42 $\mathrm{mg})$. The resulting solution was stirred under a Hydrogen balloon. After $8 \mathrm{~h}$ stirring at rt, an additional $2.5 \mathrm{wt} \%$ palladium on barium sulfate $(10.5 \mathrm{mg})$ and benzene $(7 \mathrm{ml})$ were added, and stirred an additional $2 \mathrm{~h}$ at rt. The mixture was then filtered through Celite to remove the catalyst, and the filtrate concentrated to give a yellow oil. The crude oil was purified by flash column chromatography (hexanes: ethyl acetate $=9: 1$ ) to afford $366 \mathrm{mg}$ (87 \%) of Z-allylic hemiacetal 9.

9 ; ${ }^{1} \mathrm{H}$ NMR $\left(\mathrm{C}_{6} \mathrm{D}_{6}, 400 \mathrm{MHz}\right) \delta 5.63(\mathrm{~d}, J=11.9 \mathrm{~Hz}, 1 \mathrm{H}), 5.60(\mathrm{~s}, 1 \mathrm{H}), 5.46(\mathrm{~m}, 1 \mathrm{H})$, 4.91 (m, 1H), 4.13 (m, 2H), 3.64 (m, 2H), 3.33 (s,3H), 3.32 (s, 3H), 3.05 (m, 2H), 2.65 (dd, $J=9.6 \mathrm{~Hz}, 15.4 \mathrm{~Hz}, 1 \mathrm{H}$ ), 2.24 (dd, $J=6.7 \mathrm{~Hz}, 11.3 \mathrm{~Hz}, 1 \mathrm{H}$ ), 2.17 (dd, $J=4.0 \mathrm{~Hz}$, 15.4 Hz, 1H), 1.86 (m, 3H), 1.52 (m, 1H), 1.39 (s,1H), 1.21 (d, $J=6.6 \mathrm{~Hz}, 3 \mathrm{H}$ ), 1.05 (d, $J$ = $6.9 \mathrm{~Hz}, 3 \mathrm{H}), 0.99$ (s, 9H), $0.96(\mathrm{~s}, 9 \mathrm{H}), 0.95$ (s, 9H), 0.10 (s, 3H), 0.09 (s, 3H), 0.07 (s, 3H), 0.04 (s, 3H), 0.03 (s, 3H), 0.02 (s, 3H) ; ${ }^{13} \mathrm{C} \mathrm{NMR}\left(\mathrm{C}_{6} \mathrm{D}_{6}, 400 \mathrm{MHz}\right) \delta 171.5$, 136.3, 126.8, 100.1, 83.7, 73.8, 67.8, 66.6, 59.1, 59.0, 50.8, 39.7, 39.6, 38.1, 34.4, 27.1, 26.1, 26.0, 18.3, 18.2, 12.7, 5.4, -4.1, -4.4, -4.61, -4.66, -5.1, -5.2 ; HRMS (ESI) calculated for $\mathrm{C}_{36} \mathrm{H}_{74} \mathrm{O}_{8} \mathrm{Si}_{3}(\mathrm{M}+\mathrm{Na})$ 741.4589, found 741.4592.

$\underline{\text { Preparation of } Z \text { - allylic methoxyacetal } 10}$ 


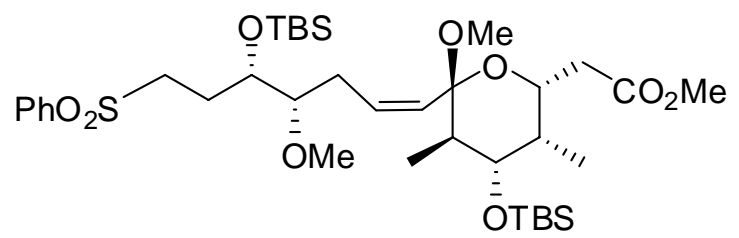

To a stirred solution of 8 (33 mg, $0.045 \mathrm{mmol})$ in $\mathrm{MeOH}(0.45 \mathrm{~mL})$ were added PPTS (pyridinium p-Toluenesulfonate, $11.3 \mathrm{mg}, 0.045 \mathrm{mmol}$ ) and $4 \mathrm{~A}^{\circ}$ molecular sieves (3 5 granule) at rt. After $10 \mathrm{~h}$ stirring at rt, the reaction was quenched with triethylamine. The mixture was filtered through Celite to remove the molecular sieves, and the filtrate was concentrated to give a yellow oil. The crude oil was purified by flash column chromatography (hexanes: ethyl acetate $=4: 1$ ) to afford $32 \mathrm{mg}$ (95\%) of Z-allylic methoxyacetal 10.

10 ; ${ }^{1} \mathrm{H}$ NMR $\left(\mathrm{C}_{6} \mathrm{D}_{6}, 400 \mathrm{MHz}\right) \delta 7.92(\mathrm{~m}, 2 \mathrm{H}), 6.97$ (m, 3H), $5.66(\mathrm{~m}, 1 \mathrm{H}), 5.45(\mathrm{~d}, J=$ $12.2 \mathrm{~Hz}, 1 \mathrm{H}), 4.50$ (td, $J=9.1 \mathrm{~Hz}, 3.2 \mathrm{~Hz}, 1 \mathrm{H}), 4.08$ (dd, $J=4.8 \mathrm{~Hz}, 10.4 \mathrm{~Hz}, 1 \mathrm{H}), 3.91$ (m, 1H), 3.37 (s, 3H), 3.29 (s, 3H), 3.24 (t, J=7.9 Hz, 2H), 3.13 (s, 3H), 3.01 (m, 1H), 2.73 (m, 3H), 2.21 (m, 1H), 2.14 (dd, $J=3.7$ Hz, 15.3 Hz, 1H), 2.02 (m, 2H), 1.84 (m, 1H), 1.22 (d, $J=6.7 \mathrm{~Hz}, 3 \mathrm{H}), 1.11$ (d, $J=6.9 \mathrm{~Hz}, 3 \mathrm{H}), 0.98$ (s, 9H), 0.87 (s, 9H), 0.08 (s, 3H), 0.04 (s, 3H), 0.03 (s, 3H), 0.00 (s, 3H) ; ${ }^{13} \mathrm{C}$ NMR $\left(\mathrm{C}_{6} \mathrm{D}_{6}, 400 \mathrm{MHz}\right) \delta 171.5$, 140.4, 133.0, 131.3, 130.8, 129.1, 103.7, 84.1, 73.1, 71.5, 58.2, 52.8, 51.1, 49.1, 41.3, 39.4, 38.3, 28.7, 26.2, 26.1, 26.0, 18.3, 18.1, 12.6, 6.1, -4.1, -4.3, -4.7 ; HRMS (ESI) calculated for $\mathrm{C}_{37} \mathrm{H}_{66} \mathrm{O}_{9} \mathrm{SSi}_{2}(\mathrm{M}+\mathrm{Na}) 765.3864$, found 765.3862.

\section{$\underline{\text { General procedure for } \mathrm{OsO}_{4}} \underline{4} \underline{\mathrm{TMEDA} / \mathrm{CH}} \underline{2}_{2} \underline{\mathrm{Cl}}_{2} \underline{3}$}

To a solution of $8(0.068 \mathrm{mmol})$ and TMEDA (N,N,N',N'-tetramethylethylenediamine, $0.075 \mathrm{mmol})$, in $\mathrm{CH}_{2} \mathrm{Cl}_{2}(6.8 \mathrm{ml})$ pre-cooled to $-78{ }^{\circ} \mathrm{C}$ under an atmosphere of $\mathrm{N}_{2}$ was added a solution of $\mathrm{OsO}_{4}(0.068 \mathrm{mmol})$ in $\mathrm{CH}_{2} \mathrm{Cl}_{2}(0.1 \mathrm{ml})$ and the reaction solution allowed to warm to rt over $3 \mathrm{~h}$. After $7 \mathrm{~h}$ stirring at rt, ethylenediamine $(0.34 \mathrm{mmol})$ was added and the reaction was then stirred for $24 \mathrm{~h}$. The mixture was poured into brine,

\footnotetext{
${ }^{3}$ Donohoe, T. J.; Newcombe, N. J.; Waring, M. J. Tetrahedron Lett. 1999, 40, 6881.
} 
extracted with ethyl acetate $(3 \times 15 \mathrm{~mL})$, dried with $\mathrm{Na}_{2} \mathrm{SO}_{4}$ and concentrated using a rotary evaporator to give a dark yellow oil. The purified oil gained near quantitative yield to afford $\mathbf{1 1} / \mathbf{1 4}$ (9:1 ratio).

\section{General procedure for catalytic osmylation $^{4}$}

To a stirred solution of $\mathbf{8}$ or $\mathbf{9}(0.068 \mathrm{mmol})$ and 4-methylmorpholine-N-oxide $(0.20$ mmol $)$ in 4:1 acetone-water $(0.68 \mathrm{ml})$ was added $\mathrm{OsO}_{4}(5 \mathrm{~mol} \%, 0.1077 \mathrm{M}$ in isopropanol) and the solution stirred at rt about $12 \mathrm{~h}$. Saturated $\mathrm{Na}_{2} \mathrm{~S}_{2} \mathrm{O}_{3}$ was then added and stirred for an additional $30 \mathrm{~min}$. The mixture was extracted with ethyl acetate $(3 \times 15$ $\mathrm{mL}$ ), dried with $\mathrm{Na}_{2} \mathrm{SO}_{4}$ and concentrated using a rotary evaporator to give a dark yellow oil. The purified oil gained $>95 \%$ yield to afford 11/14 (3:1 ratio) or 12/15 (4:1 ratio).

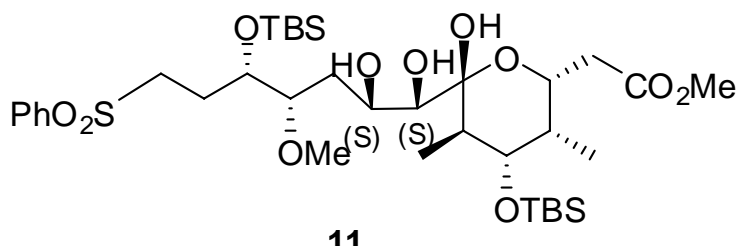

11

11 ; ${ }^{1} \mathrm{H}$ NMR $\left(\mathrm{C}_{6} \mathrm{D}_{6}, 400 \mathrm{MHz}\right) \delta 7.90$ (m, 2H), 6.99 (3s, 3H), 4.86 (s, 1H), 4.78 (m, 1H), 4.19 (dd, $J=4.6 \mathrm{~Hz}, 10.1 \mathrm{~Hz}, 2 \mathrm{H}$ ), 3.98 (m, 1H), 3.82 (dd, $J=4.7 \mathrm{~Hz}, 10.1 \mathrm{~Hz}, 1 \mathrm{H}$ ), 3.55 (m, 1H), 3.49 (s, 3H), 3.19 (s, 3H), 3.18 (t, $J=7.8 \mathrm{~Hz}, 2 \mathrm{H}), 2.64$ (dd, $J=9.9 \mathrm{~Hz}$, 15.7 Hz, 1H), 2.19 (dd, $J=3.5$ Hz, 15.6 Hz, 1H), 2.13 (m, 2H), 1.95 (m, 4H), 1.23 (d, $J=$ $6.5 \mathrm{~Hz}, 3 \mathrm{H}), 1.09$ (d, $J=6.8 \mathrm{~Hz}, 3 \mathrm{H}), 0.96$ (s, 9H), 0.88 (s, 9H), 0.08 (s, 3H), 0.07 (s, 3H), 0.03 (s, 3H), 0.01 (s, 3H) ; ${ }^{13} \mathrm{C}$ NMR $\left(\mathrm{C}_{6} \mathrm{D}_{6}, 400 \mathrm{MHz}\right) \delta 172.1,140.2,133.2$, 129.1, 128.5, 100.3, 81.1, 76.9, 73.4, 70.6, 69.6, 67.6, 58.6, 52.6, 51.6, 39.7, 37.8, 37.4, 32.9, 26.1, 26.0, 18.2, 18.1, 12.3, 5.9, -4.1, -4.3, -4.6, -4.7. ; HRMS (ESI) calculated for $\mathrm{C}_{36} \mathrm{H}_{66} \mathrm{O}_{11} \mathrm{SSi}_{2}(\mathrm{M}+\mathrm{Na}) 785.3762$, found 785.3773; mp $=125.4-128.4{ }^{\circ} \mathrm{C}$; the structure was confirmed via X-ray analysis (See attached cif file).

${ }^{4}$ (a) Cha, J. K.; Christ, W. J.; Kishi, Y. Tetrahedron Lett. 1983, 24, 3943. (b) Cha, J. K.; No-Soo, K. Chem. Rev. 1995, 95, 1761. 


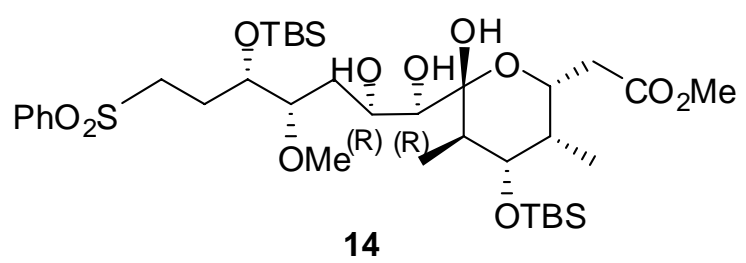

14 ; ${ }^{1} \mathrm{H}$ NMR $\left(\mathrm{C}_{6} \mathrm{D}_{6}, 400 \mathrm{MHz}\right) \delta 7.92(\mathrm{~m}, 2 \mathrm{H}), 6.96(\mathrm{~m}, 3 \mathrm{H}), 5.99(\mathrm{~d}, J=0.8 \mathrm{~Hz}, 1 \mathrm{H})$, 4.88 (m, 1H), 4.27 (d, $J=1.8 \mathrm{~Hz}, 1 \mathrm{H}$ ), 4.18 (dd, $J=4.7 \mathrm{~Hz}, 10.4 \mathrm{~Hz}, 2 \mathrm{H}), 4.09$ (m, 1H), 3.51 (m, 1H), 3.40 (s, 3H), 3.34 (m, 1H), 3.15 (m, 1H), 3.04 (s, 3H), 2.55 (dd, $J=9.6$ Hz, 15.0 Hz, 1H), 2.42 (m, 1H), 2.26 (m, 1H), 2.14 (dd, $J=4.5$ Hz, 15.1 Hz, 1H), 1.85 (m, 1H), 1.74 (m, 2H), 1.41 (m, 1H), 1.31 (d, $J=6.6 \mathrm{~Hz}, 3 \mathrm{H}), 0.99$ (s, 9H), 0.90 (d, $J=$ $6.6 \mathrm{~Hz}, 3 \mathrm{H}), 0.87$ (s, 9H), 0.10 (s, 3H), 0.04 (s, 3H), 0.03 (s, 6H) ; ${ }^{13} \mathrm{C}$ NMR $\left(\mathrm{C}_{6} \mathrm{D}_{6}, 400\right.$ MHz) $\delta 171.7,140.3,133.2,129.1,128.2,101.8,84.7,74.0,73.3,73.0,69.5,67.2,57.4$, 52.4, 51.0, 39.3, 37.7, 36.1, 32.8, 26.0, 25.8, 18.3, 18.0, 12.2, 5.3, -4.1, -4.4, -4.6, -4.7. ; HRMS (ESI) calculated for $\mathrm{C}_{36} \mathrm{H}_{66} \mathrm{O}_{11} \mathrm{SSi}_{2}(\mathrm{M}+\mathrm{Na})$ 785.3762, found 785.3759.

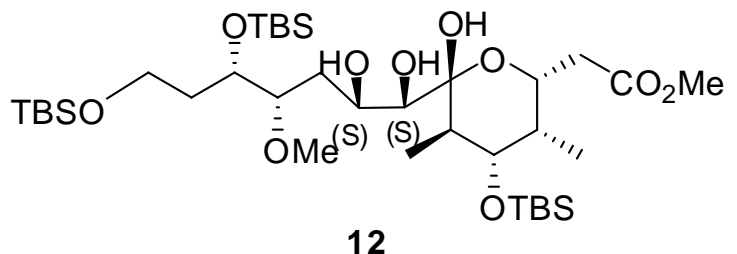

$12 ;{ }^{1} \mathrm{H}$ NMR $\left(\mathrm{C}_{6} \mathrm{D}_{6}, 400 \mathrm{MHz}\right) \quad \delta 4.76(\mathrm{~m}, 1 \mathrm{H}), 4.56(\mathrm{~s}, 1 \mathrm{H}), 4.27$ (m,2H), 4.15 (dd, $J=$ $4.7 \mathrm{~Hz}, 10.3 \mathrm{~Hz}, 1 \mathrm{H}), 3.74$ (m, 3H), 3.38 (s, 3H), 3.31 (d, $J=8.2 \mathrm{~Hz}, 1 \mathrm{H}), 3.23$ (s, 3H), 3.15 (d, $J=9.8 \mathrm{~Hz}, 1 \mathrm{H}$ ), 2.54 (dd, $J=9.6 \mathrm{~Hz}, 14.9 \mathrm{~Hz}, 1 \mathrm{H}$ ), 2.27 (m, 1H), 2.14 (dd, $J=$ $4.3 \mathrm{~Hz}, 14.9 \mathrm{~Hz}, 1 \mathrm{H}), 2.08$ (m, 1H), 1.96 (m, 1H), 1.88 (m, 1H), 1.58 (m, 1H), 1.19 (d, $J$ = 6.6 Hz, 3H), $1.06(\mathrm{~J}=6.9 \mathrm{~Hz}, 3 \mathrm{H}), 1.01(\mathrm{~s}, 9 \mathrm{H}), 0.98$ (s, 9H), 0.94 (s, 9H), 0.19 (s, 3H), 0.15 (s, 3H), 0.07 (s, 3H), 0.06 (s, 3H), 0.05 (s, 3H), 0.01 (s, 3H) ; ${ }^{13} \mathrm{C}$ NMR $\left(\mathrm{C}_{6} \mathrm{D}_{6}, 400\right.$ MHz) $\delta 171.5,100.8,81.5,75.5,73.4,70.4,67.8,67.4,59.5,57.6,51.2,39.5,37.8$, 37.6, 34.6, 33.4, 26.2, 26.1, 26.0, 18.3, 12.4, 5.9, -4.2, -4.6, -4.7, -5.1, -5.2 ; HRMS (ESI) calculated for $\mathrm{C}_{36} \mathrm{H}_{76} \mathrm{O}_{10} \mathrm{Si}_{3}(\mathrm{M}+\mathrm{Na})$ 775.4644, found 775.4645. 


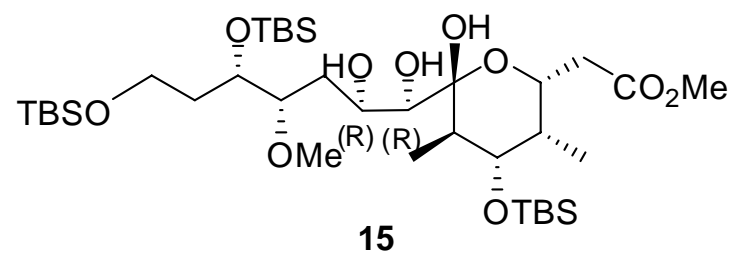

15 ; ${ }^{1} \mathrm{H}$ NMR $\left(\mathrm{C}_{6} \mathrm{D}_{6}, 400 \mathrm{MHz}\right) \quad \delta 6.21(\mathrm{~s}, 1 \mathrm{H}), 4.94(\mathrm{~m}, 1 \mathrm{H}), 4.44(\mathrm{~s}, 1 \mathrm{H}), 4.30(\mathrm{t}, J=$ $8.9 \mathrm{~Hz}, 1 \mathrm{H}), 4.20$ (dd, $J=4.7 \mathrm{~Hz}, 10.7 \mathrm{~Hz}, 1 \mathrm{H}), 3.72$ (m, 2H), 3.61 (dd, $J=9.2 \mathrm{~Hz}, 10.4$ Hz, 1H), 3.53 (m, 1H), 3.41 (s, 3H), 3.06 (s, 3H), 2.57 (m, 2H), 2.42 (m 1H), 2.19 (dd, J = $5.1 \mathrm{~Hz}, 14.9 \mathrm{~Hz}, 1 \mathrm{H}), 1.89(\mathrm{~m}, 1 \mathrm{H}), 1.78$ (d, J=10.8 Hz, 1H), 1.61 (m, 1H), 1.49 (m, 1H), 1.32 (d, $J=6.6 \mathrm{~Hz}, 3 \mathrm{H}), 0.99$ (s, 9H), 0.97 (s, 9H), 0.96 (s, 9H), $0.90(J=6.8 \mathrm{~Hz}$, 3H), 0.12 (s, 3H), 0.094 (s, 3H), 0.091 (s, 3H), 0.06 (s, 3H), 0.05 (s, 3H), 0.03 (s, 3H) ; ${ }^{13} \mathrm{C}$ NMR $\left(\mathrm{C}_{6} \mathrm{D}_{6}, 500 \mathrm{MHz}\right) \quad \delta 171.4,101.9,85.5,74.7,73.3,73.0,67.3,66.8,59.2,56.8$, 51.0, 39.3, 37.9, $36.2, \quad 34.2, \quad 32.9, \quad 26.0, \quad 25.9, \quad 18.3, \quad 18.2, \quad 12.2$, 5.3, -4.1, -4.2, -4.6, -4.7, -5.2, -5.3; HRMS (ESI) calculated for $\mathrm{C}_{36} \mathrm{H}_{76} \mathrm{O}_{10} \mathrm{Si}_{3}(\mathrm{M}+\mathrm{Na})$ 775.4644, found 775.4647 .

\section{Preparation of E-allylic hemiacetal 17}

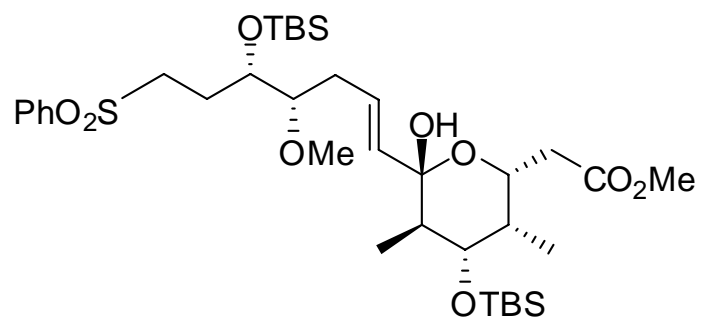

To a stirred solution of 8 (55 $\mathrm{mg}, 0.075 \mathrm{mmol})$ in toluene $(2.51 \mathrm{~mL})$ were added quinoline (35.6 $\mu \mathrm{l}, 0.30 \mathrm{mmol})$ and thiophenol $(2.32 \mu \mathrm{l}, 0.022 \mathrm{mmol})$. The resulting solution was heated to $60{ }^{\circ} \mathrm{C}$ and stirred at $60{ }^{\circ} \mathrm{C}$ over $1 \sim 1.5$ day, when two more portions of thiophenol $(2 \times 22.32 \mu \mathrm{l}, 0.044 \mathrm{mmol})$ were added at an interval of $8 \mathrm{~h}$. The reaction solution was concentrated to give a crude brown oil that was purified by flash column 
chromatography (hexanes: ethyl acetate $=7: 3$ ) to afford $44 \mathrm{mg}(80 \%)$ of $E$-allylic hemiacetal 17.

$17 ;{ }^{1} \mathrm{H}$ NMR $\left(\mathrm{C}_{6} \mathrm{D}_{6}, 300 \mathrm{MHz}\right) \quad \delta 7.86(\mathrm{~m}, 2 \mathrm{H}), 6.96(3 \mathrm{~s}, 3 \mathrm{H}), 5.87(\mathrm{~m}, 1 \mathrm{H}), 5.62(\mathrm{~d}, J=$ $15.9 \mathrm{~Hz}, 1 \mathrm{H}), 4.84$ (m, 1H), 4.10 (dd, $J=5.1 \mathrm{~Hz}, 10.6 \mathrm{~Hz}, 1 \mathrm{H}), 3.87$ (m, 1H), 3.35 (s, 3H), 3.18 (m, 2H), 3.08 (s, 3H), 2.93 (m, 1H), 2.67 (dd, J = 9.6 Hz, $15.6 \mathrm{~Hz}, 1 \mathrm{H}), 2.17$ (dd, $J=3.9 \mathrm{~Hz}, 15.0 \mathrm{~Hz}, 1 \mathrm{H}), 2.08$ (m, 2H), 1.85 (m, 3H), 1.71 (m, 1H), 1.19 (d, $J=7.2$ Hz, 3H), 1.01 (d, $J=6.3$ Hz, 3H), 0.98 (s, 9H), 0.87 (s, 9H), 0.07 (s, 3H), 0.03 (s, 6H), 0.00 (s, 3H) ; ${ }^{13} \mathrm{C}$ NMR $\left(\mathrm{C}_{6} \mathrm{D}_{6}, 400 \mathrm{MHz}\right) \quad \delta 171.6,140.2,136.0,133.1,129.1,127.4$, 99.0, 83.4, 73.7, 71.1, 67.7, 57.7, 52.7, 51.1, 39.5, 39.4, 37.9, 31.8, 26.0, 25.9, 18.2, 18.1, 12.4, 5.3, -4.1, -4.3, -4.7 ; HRMS (ESI) calculated for $\mathrm{C}_{36} \mathrm{H}_{64} \mathrm{O}_{9} \mathrm{SSi}_{2}(\mathrm{M}+\mathrm{Na}) 751.3707$, found 751.3716 .

Preparation of E-allylic hemiacetal 18

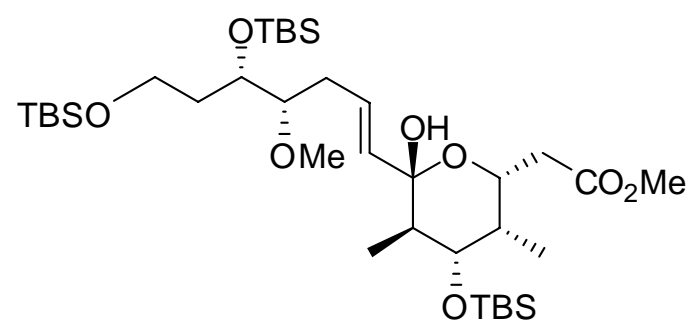

To a stirred solution of 9 (60 $\mathrm{mg}, 0.083 \mathrm{mmol})$ in toluene $(0.27 \mathrm{~mL})$ were added quinoline $(39.4 \mu \mathrm{l}, 0.33 \mathrm{mmol})$ and thiophenol $(2.57 \mu \mathrm{l}, 0.025 \mathrm{mmol})$. The resulting solution was heated to $60{ }^{\circ} \mathrm{C}$ and stirred at $60{ }^{\circ} \mathrm{C}$ over $1 \sim 1.5$ day, when two more portions of thiophenol $(2 \times 2.57 \mu \mathrm{l}, 0.050 \mathrm{mmol})$ were added at an interval of $8 \mathrm{~h}$. The reaction solution was concentrated to give a crude brown oil that was purified by flash column chromatography (hexanes: ethyl acetate $=4: 1$ ) to afford $49 \mathrm{mg}(83 \%)$ of E-allylic hemiacetal 18.

$18 ;{ }^{1} \mathrm{H}$ NMR $\left(\mathrm{C}_{6} \mathrm{D}_{6}, 400 \mathrm{MHz}\right) \quad \delta 5.96(\mathrm{~m}, 1 \mathrm{H}), 5.70(\mathrm{~d}, J=15.5 \mathrm{~Hz}, 1 \mathrm{H}), 4.82(\mathrm{~m}, 1 \mathrm{H})$, 4.16 (m, 2H), 4.07 (dd, J = 4.8 Hz, 10.5 Hz, 1H), 3.81 (m,1H), 3.33 (s,3H), 3.21 (s, 3H), 3.20 (m, 1H), 2.65 (dd, $J=9.0 \mathrm{~Hz}, 15.6 \mathrm{~Hz}, 1 \mathrm{H}), 2.48$ (m, 1H), 2.21 (m, 2H), 1.97 
(s,1H), $1.94(\mathrm{~m}, 1 \mathrm{H}), 1.86(\mathrm{~m}, 2 \mathrm{H}), 1.60(\mathrm{~m}, 1 \mathrm{H}), 1.20(\mathrm{~d}, J=6.7 \mathrm{~Hz}, 3 \mathrm{H}), 1.00(\mathrm{~d}, J=$ $6.9 \mathrm{~Hz}, 3 \mathrm{H}$ ), 0.99 (s, 9H), 0.98 (s, 9H), 0.97 (s, 9H), 0.14 (s, 3H), 0.12 (s, 3H), 0.08 (s, 3H), 0.07 (s, 3H), $0.06(\mathrm{~s}, 3 \mathrm{H}), 0.01(\mathrm{~s}, 3 \mathrm{H}) ;{ }^{13} \mathrm{C}$ NMR $\left(\mathrm{C}_{6} \mathrm{D}_{6}, 400 \mathrm{MHz}\right) \quad \delta 171.8$, 135.5, 128.4, 99.2, 83.9, 73.7, 68.7, 67.6, 59.6, 57.5, 51.1, 39.6, 39.5, 37.9, 34.9, 31.9, 26.1, 26.0, 18.8, 18.2, 12.4, 5.4, -4.1, -4.5, -4.7, -5.14, -5.19 . ; HRMS (ESI) calculated for $\mathrm{C}_{36} \mathrm{H}_{74} \mathrm{O}_{8} \mathrm{Si}_{3}(\mathrm{M}+\mathrm{Na})$ 741.4589, found 741.4597 .

\section{Preparation of E-allylic methoxy acetal 19}

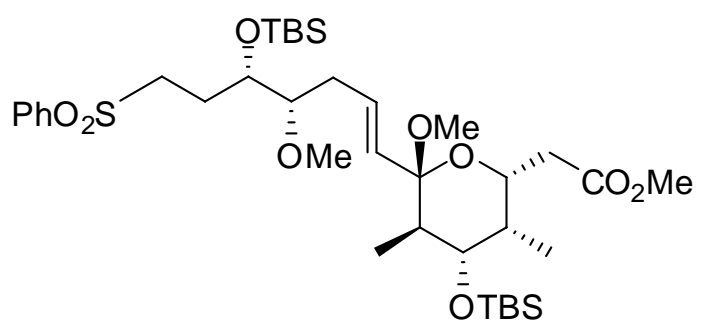

To a stirred solution of 17 (33 mg, $0.045 \mathrm{mmol})$ in $\mathrm{MeOH}(0.45 \mathrm{~mL}$ ) were added PPTS (pyridinium $p$-Toluenesulfonate, $11.3 \mathrm{mg}, 0.045 \mathrm{mmol}$ ) and $4 \mathrm{~A}^{0}$ molecular sieves (3 5 granule) at rt. After $10 \mathrm{~h}$ stirring at rt, the reaction was quenched with triethylamine. The mixture filtered through Celite to remove the molecular sieves, and the filtrate was concentrated to give a yellow oil. The crude oil was purified by flash column chromatography (hexanes: ethyl acetate $=7: 3$ ) to afford $32 \mathrm{mg}(95 \%)$ of $E$-allylic methoxy acetal_19.

$19 ;{ }^{1} \mathrm{H}$ NMR $\left(\mathrm{C}_{6} \mathrm{D}_{6}, 400 \mathrm{MHz}\right) \quad \delta 7.86(\mathrm{~m}, 2 \mathrm{H}), 6.96$ (3s, 3H), 6.09 (td, $J=7.1 \mathrm{~Hz}, 15.6$ Hz, 1H), 5.56 (d, J=15.6 Hz, 1H), 4.50 (m, 1H), 4.09 (dd, $J=4.8$ Hz, 10.4 Hz, 1H), 3.91 (m, 1H), 3.34 (s, 3H), 3.29 (s, 3H), 3.15 (m, 2H), 3.09 (s, 3H), 2.96 (m, 1H), 2.66 (dd, $J=$ $9.6 \mathrm{~Hz}, 15.4 \mathrm{~Hz}, 1 \mathrm{H}), 2.26$ (m, 1H), 2.19 (m, 1H), 2.10 (dd, $J=3.9 \mathrm{~Hz}, 15.2 \mathrm{~Hz}, 1 \mathrm{H}$ ), 1.96 (m, 1H), 1.81 (m, 3H), 1.25 (d, $J=6.7 \mathrm{~Hz}, 3 \mathrm{H}), 0.99$ (d, $J=6.7 \mathrm{~Hz}, 3 \mathrm{H}), 0.97$ (s, 9H), 0.87 (s, 9H), 0.08 (s, 3H), 0.02 (s, 6H), 0.00 (s, 3H) ; ${ }^{13} \mathrm{C}$ NMR $\left(\mathrm{C}_{6} \mathrm{D}_{6}, 400 \mathrm{MHz}\right)$ $\delta$ 171.5, 140.3, 133.0, 131.8, 130.2, 129.1, 101.7, 83.7, 73.4, 70.9, 67.9, 57.9, 52.8, 51.0, 
49.0, 41.5, 39.4, 38.0, 32.1, 26.0, 25.9, 18.2, 18.1, 12.2, 5.6, -4.1, -4.4, -4.6, -4.7 ; HRMS (ESI) calculated for $\mathrm{C}_{37} \mathrm{H}_{66} \mathrm{O}_{9} \mathrm{SSi}_{2}(\mathrm{M}+\mathrm{Na})$ 765.3864, found 765.3880.

\section{$\underline{\mathrm{OsO}}_{4} / \underline{\mathrm{TMEDA}}_{\mathrm{CH}} \underline{\mathrm{Cl}}_{2} \underline{\mathrm{C}_{2} \text { conditions }}$}

The procedure was same as used with 8 .

Preparation of 20/23: The ${ }^{1} \mathrm{H}$ NMR of the crude oil shows $>95 \%$ conversion to afford 20 /23 (5:1 ratio).

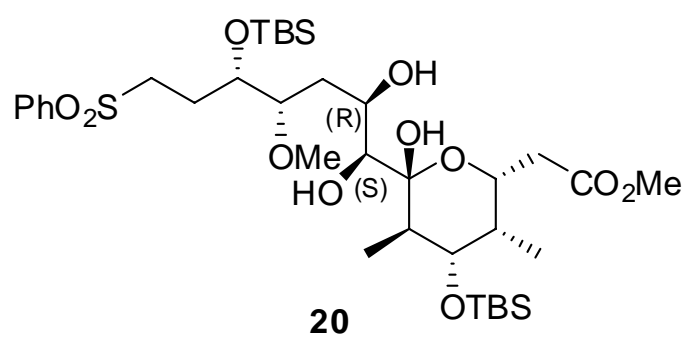

20 ; ${ }^{1} \mathrm{H}$ NMR $\left(\mathrm{C}_{6} \mathrm{D}_{6}, 300 \mathrm{MHz}\right) \quad \delta 7.85(\mathrm{~m}, 2 \mathrm{H}), 6.95(\mathrm{~m}, 3 \mathrm{H}), 4.66(\mathrm{~m}, 1 \mathrm{H}), 4.47$ (s, 1H), 4.32 (t, $J=6.6 \mathrm{~Hz}, 1 \mathrm{H}), 4.14$ (m, 1H), 4.05 (dd, $J=4.5 \mathrm{~Hz}, 9.9 \mathrm{~Hz}, 2 \mathrm{H}$ ), 3.70 (s, 1H), 3.56 (d, $J=9.9 \mathrm{~Hz}, 1 \mathrm{H}$ ), 3.33 (s, 3H), 3.29 (m, 1H), 3.16 (m, 1H), 3.13 (s, 3H), 2.36 (dd, $J$ = 9.3 Hz, $14.7 \mathrm{~Hz}, 1 \mathrm{H}), 2.21$ (m, 1H), 2.02 (dd, $J=4.2 \mathrm{~Hz}, 14.7 \mathrm{~Hz}, 1 \mathrm{H}), 1.93$ (m, 4H), 1.19 (d, $J=6.9 \mathrm{~Hz}, 3 \mathrm{H}$ ), 0.95 (s, 9H), 0.90 (s, 9H), 0.87 (d, $J=7.2 \mathrm{~Hz}, 3 \mathrm{H}$ ), 0.08 (s, 3H), 0.04 (s, 6H), -0.01 (s, 3H) ; ${ }^{13} \mathrm{C}$ NMR $\left(\mathrm{C}_{6} \mathrm{D}_{6}, 300 \mathrm{MHz}\right) \quad \delta 171.3,140.3,133.1,129.1$, 128.3, 101.1, 81.4, 73.3, 70.2, 67.8, 67.3, 57.4, 52.7, 51.3, 39.3, 37.7, 37.0, 32.8, 26.1, 26.0, 18.2, 18.1, 12.5, 5.3, -4.2, -4.3, -4.5, -4.8. ; HRMS (ESI) calculated for $\mathrm{C}_{36} \mathrm{H}_{66} \mathrm{O}_{11} \mathrm{SSi}_{2}(\mathrm{M}+\mathrm{Na})$ 785.3762, found 785.3769. 


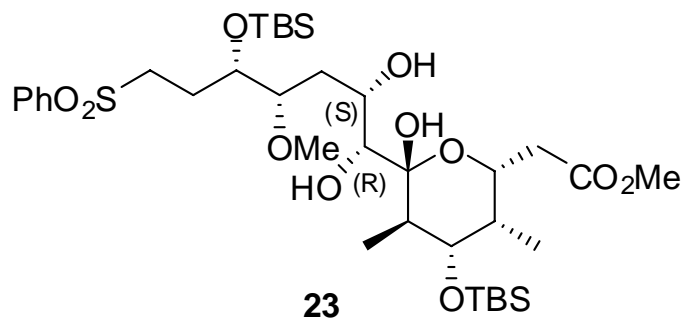

23 ; ${ }^{1} \mathrm{H}$ NMR $\left(\mathrm{C}_{6} \mathrm{D}_{6}, 300 \mathrm{MHz}\right) \quad \delta 7.90(\mathrm{~m}, 2 \mathrm{H}), 6.95(\mathrm{~m}, 3 \mathrm{H}), 4.49(\mathrm{tt}, J=9.9 \mathrm{~Hz}, 2.4$ Hz, 1H), 4.14 (quint, $J=4.5 \mathrm{~Hz}, 1 \mathrm{H}$ ), 3.88 (dd, $J=4.5 \mathrm{~Hz}, 10.5 \mathrm{~Hz}, 1 \mathrm{H}$ ), 3.70 (m, 1H), 3.27 (d, $J=15.3 \mathrm{~Hz}, 1 \mathrm{H}), 3.27$ (t, $J=6.9 \mathrm{~Hz}, 1 \mathrm{H}), 3.01$ (d, $J=10.5 \mathrm{~Hz}, 1 \mathrm{H}), 2.58$ (dd, $J=$ $10.8 \mathrm{~Hz}, 7.2 \mathrm{~Hz}, 1 \mathrm{H}), 2.34$ (m, 3H), 1.96 (dd, $J=3$ Hz, 14.7 Hz, 1H), 1.87 (m, 1H), 1.70 (m, 1H), 1.44 (m, 2H), 1.15 (d, $J=6.6 \mathrm{~Hz}, 3 \mathrm{H}), 0.97$ (s, 9H), 0.92 (s, 9H), 0.85 (d, $J=7.3$ $\mathrm{Hz}, 3 \mathrm{H}), 0.16$ (s, 3H), 0.07 (s, 3H), 0.06 (s, 3H), 0.02 (s, 3H) ; ${ }^{13} \mathrm{C}$ NMR $\left(\mathrm{C}_{6} \mathrm{D}_{6}, 300\right.$ MHz) $\delta 172.2,140.3,133.1,129.1,128.3,102.9,80.4,73.9,73.0,70.3,68.5,65.5,58.6$, 52.9, 51.4, 39.5, 37.8, 35.3, 33.9, 25.9, 26.0, 18.2, 18.1, 12.1, 5.2, -4.1, -4.2, -4.6, -4.7 ; LRMS (ESI) calculated for $\mathrm{C}_{36} \mathrm{H}_{66} \mathrm{O}_{11} \mathrm{SSi}_{2}(\mathrm{M}+\mathrm{Na})$ 785.37, found 785.27.

Preparation of 22/25: The ${ }^{1} \mathrm{H}$ NMR of the crude oil shows $80 \%$ conversion to afford 22 /25 (1:7 ratio).

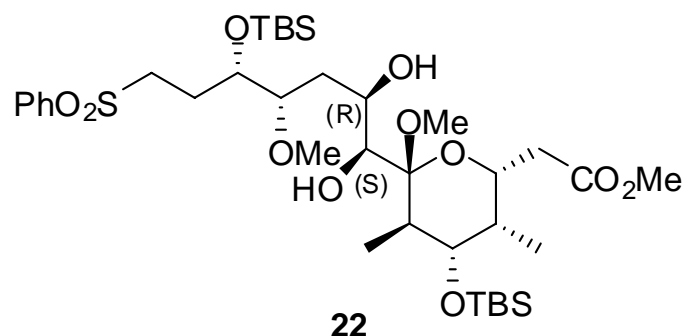

22 ; ${ }^{1} \mathrm{H}$ NMR $\left(\mathrm{C}_{6} \mathrm{D}_{6}, 300 \mathrm{MHz}\right) \quad \delta 7.81$ (m, 2H), 6.95 (m, 3H), 4.37 (m, 1H), 4.18 (m, 2H), 4.06 (dd, $J=5.1 \mathrm{~Hz}, 9.9 \mathrm{~Hz}, 1 \mathrm{H}), 3.72$ (d, $J=9.9 \mathrm{~Hz}, 1 \mathrm{H}), 3.58$ (s, 3H), 3.32 (s, 3H), 3.29 (m, 1H), 3.19 (m, 1H), 3.08 (s, 3H), 3.09 (m, 1H), 2.54 (dd, J = 10.2 Hz, 15.3 Hz, 1H), 2.21 (m, 2H), 2.03 (dd, $J=3.6 \mathrm{~Hz}, 15.3 \mathrm{~Hz}, 1 \mathrm{H}), 1.88$ (m, 2H), 1.78 (m, 2H), 1.19 (d, $J=6.6 \mathrm{~Hz}, 3 \mathrm{H}), 1.02$ (d, $J=6.9 \mathrm{~Hz}, 3 \mathrm{H}), 0.95$ (s, 9H), 0.88 (s, 9H), 0.06 (s, 3H), 0.04 (s, 3H), 0.03 (s, 3H), 0.00 (s, 3H) ; ${ }^{13} \mathrm{C}$ NMR $\left(\mathrm{C}_{6} \mathrm{D}_{6}, 300 \mathrm{MHz}\right) \delta 171.4,140.3,133.1$, 
129.1, 128.3, 102.1, 82.5, 75.8, 73.7, 69.7, 68.9, 57.1, 52.6, 51.1, 39.3, 37.8, 37.3, 32.6, 26.0, 18.2, 18.1, 12.3, 5.6, -4.1, -4.4, -4.6, -4.7 ; LRMS (ESI) calculated for $\mathrm{C}_{37} \mathrm{H}_{68} \mathrm{O}_{11} \mathrm{SSi}_{2}(\mathrm{M}+\mathrm{Na})$ 799.39, found 799.16 .

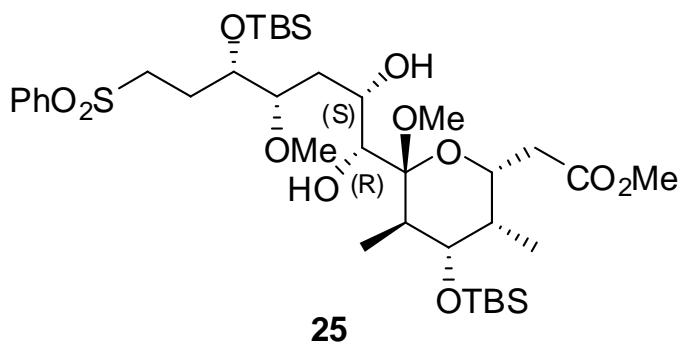

$25:{ }^{1} \mathrm{H}$ NMR $\left(\mathrm{C}_{6} \mathrm{D}_{6}, 300 \mathrm{MHz}\right) \quad \delta 7.87(\mathrm{~m}, 2 \mathrm{H}), 6.94(\mathrm{~m}, 3 \mathrm{H}), 4.28(\mathrm{td}, J=9.3 \mathrm{~Hz}, 2.7$ Hz, 1H), 4.06 (quint, $J=4.5 \mathrm{~Hz}, 1 \mathrm{H}$ ), 3.97 (dd, $J=4.5 \mathrm{~Hz}, 10.5 \mathrm{~Hz}, 1 \mathrm{H}$ ), 3.62 (m, 1H), 3.55 (dd, $J=1.2 \mathrm{~Hz}, 6.3 \mathrm{~Hz}, 1 \mathrm{H}$ ), 3.34 (s, 3H), 3.26 (s, 3H), 3.24 (s, 3H), 3.21 (m, 2H), 2.95 (d, $J=5.4 \mathrm{~Hz}, 1 \mathrm{H}), 2.64$ (d, $J=6.3 \mathrm{~Hz}, 1 \mathrm{H}), 2.47$ (dt, $J=4.8 \mathrm{~Hz}, 9.9 \mathrm{~Hz}, 1 \mathrm{H}$ ), 2.22 (m, 1H), 1.98 (dd, $J=3.6 \mathrm{~Hz}, 15.3 \mathrm{~Hz}, 1 \mathrm{H}), 1.86$ (m, 1H), 1.71 (m, 1H), 1.48 (m, 1H), 1.31 (d, $J=6.9 \mathrm{~Hz}, 1 \mathrm{H}$ ), 0.96 (s, 9H), 0.94 (d, $J=6.9 \mathrm{~Hz}, 3 \mathrm{H}$ ), 0.90 (s, 9H), 0.12 (s, 3H), 0.07 (s, 3H), 0.03 (s, 3H), 0.01 (s, 3H) ; ${ }^{13} \mathrm{C}$ NMR $\left(\mathrm{C}_{6} \mathrm{D}_{6}, 300 \mathrm{MHz}\right) \delta$ 171.6, 140.4, 133.0, 129.1, 128.3, 103.6, 80.5, 74.9, 73.4, 70.0, 69.0, 67.4, 58.3, 53.0, 51.1, 48.6, 39.1, 37.9, 36.5, 34.6, 26.0, 18.2, 18.1, 12.4, 5.6, -4.1, -4.4, -4.6 ; HRMS (ESI) calculated for $\mathrm{C}_{37} \mathrm{H}_{68} \mathrm{O}_{11} \mathrm{SSi}_{2}(\mathrm{M}+\mathrm{Na})$ 799.3919, found 799.3907.

\section{Catalytic osmylation conditions}

The procedure was same as used with $\mathbf{8 , 9}$.

Preparation of 20/23 : The ${ }^{1} \mathrm{H}$ NMR of the crude oil shows $>90 \%$ conversion to afford 20/23 (2:1 ratio).

\section{Preparation of 22/25:}

To a stirred solution of 19 (0.068 mmol) and 4-methylmorpholine-N-oxide (0.20 mmol) in 4:1 acetone-water $(0.68 \mathrm{ml})$ was added $\mathrm{OsO}_{4}(5 \mathrm{~mol} \%, 0.1077 \mathrm{M}$ in isopropanol) and 
the solution stirred at rt for $12 \mathrm{~h}$. After adding additional 4-methylmorpholine-N-oxide (0.20 mmol), $\mathrm{OsO}_{4}$ (5 mol \%, $0.1077 \mathrm{M}$ in isopropanol), the solution mixture stirred for an $36 \mathrm{~h}$ at rt. Saturated $\mathrm{Na}_{2} \mathrm{~S}_{2} \mathrm{O}_{3}$ solution was added and stirred for an additional $30 \mathrm{~min}$. The mixture was extracted with ethyl acetate $(3 \times 15 \mathrm{~mL})$, dried with $\mathrm{Na}_{2} \mathrm{SO}_{4}$ and concentrated using a rotary evaporator to give a dark yellow oil. The ${ }^{1} \mathrm{H}$ NMR of the crude oil shows $\sim 50 \%$ conversion to afford 22/25 (1:2 ratio ).

Preparation of 21/24 : The ${ }^{1} \mathrm{H}$ NMR of the crude oil shows $>90 \%$ conversion to afford 21/24 (2.5:1 ratio ).

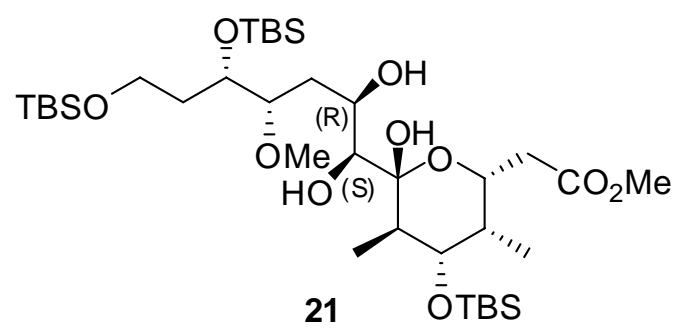

21 ; ${ }^{1} \mathrm{H}$ NMR $\left(\mathrm{C}_{6} \mathrm{D}_{6}, 400 \mathrm{MHz}\right) \quad \delta 4.67$ (m, 1H), 4.43 (m, 1H), 4.41 (d, J=1.1 Hz, 1H), 4.30 (m, 1H), 4.05 (dd, $J=4.7 \mathrm{~Hz}, 10.2 \mathrm{~Hz}, 1 \mathrm{H}$ ), 3.76 (m, 1H), 3.70 (s, 1H), 3.71 (m, 1H), 3.51 (m, 1H), 3.33 (s, 3H), 3.25 (d, $J=9.9 H z, 1 H$ ), 3.21 (s, 3H), 2.36 (dd, $J=9.5$ Hz, $14.4 \mathrm{~Hz}, 1 \mathrm{H}$ ), 2.18 (ddd, $J=3.2 \mathrm{~Hz}, 6.6 \mathrm{~Hz}, 14.2 \mathrm{~Hz}, 1 \mathrm{H}$ ), 2.03 (dd, $J=5.0 \mathrm{~Hz}$, 14.4 Hz, 1H), 1.96 (m, 3H), 1.78 (m, 1H), 1.59 (m, 1H), 1.24 (d, J = 6.5 Hz, 3H), 1.01 (s, 9H), 0.98 (s, 9H), 0.96 (s, 9H), 0.88 (d, $J=6.9 \mathrm{~Hz}, 3 \mathrm{H}), 0.167$ (s, 3H), 0.16 (s, 3H), 0.07 (s, 3H), 0.06 (s, 3H), 0.04 (s, 3H), 0.01 (s, 3H) ; ${ }^{13} \mathrm{C}$ NMR $\left(\mathrm{C}_{6} \mathrm{D}_{6}, 400 \mathrm{MHz}\right) \delta$ 171.2, 101.2, 81.5, 73.4, 73.2, 67.8, 67.5, 59.5, 57.0, 51.2, 39.3, 37.8, 37.1, 34.6, 32.8, 26.1, 26.0, 18.3, 18.2, 12.5, 5.3, -4.1, -4.5, -4.8, -5.1, -5.2 ; HRMS (ESI) calculated for $\mathrm{C}_{36} \mathrm{H}_{76} \mathrm{O}_{10} \mathrm{Si}_{3}(\mathrm{M}+\mathrm{Na})$ 775.4644, found 775.4650. 


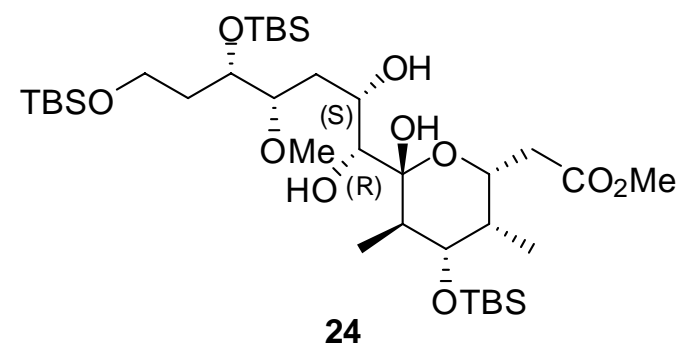

24 ; ${ }^{1} \mathrm{H}$ NMR $\left(\mathrm{C}_{6} \mathrm{D}_{6}, 500 \mathrm{MHz}\right) \quad \delta 4.45(\mathrm{~m}, 1 \mathrm{H}), 4.36(\mathrm{~m}, 1 \mathrm{H}), 3.88$ (dd, $J=4.7 \mathrm{~Hz}, 10.7$ Hz, 1H), 3.83 (m, 2H), 3.50 (t, J=10.7 Hz, 2H), 3.415 (s, 3H), 3.411 (s, 3H), 2.98 (d, J = $10.4 \mathrm{~Hz}, 1 \mathrm{H}), 2.79$ (s, 1H), 2.54 (m, 1H), 2.42 (dt, $J=2.0 \mathrm{~Hz}, 13.6 \mathrm{~Hz}, 1 \mathrm{H}), 2.30$ (dd, $J$ = $10.4 \mathrm{~Hz}, 14.6 \mathrm{~Hz}, 1 \mathrm{H}), 2.04$ (m, 1H), 1.92 (dd, $J=3.4 \mathrm{~Hz}, 14.6 \mathrm{~Hz}, 1 \mathrm{H}), 1.66$ (m, 3H), 1.10 (d, $J=6.6 \mathrm{~Hz}, 3 \mathrm{H}), 1.04$ (s, 9H), 1.00 (s, 9H), 0.96 (s, 9H), 0.82 (d, $J=6.9$ Hz, 3H), 0.25 (s, 3H), 0.20 (s, 3H), 0.10 (s, 3H), 0.09 (s, 3H), 0.02 (s, 3H), 0.01 (s, 3H) ; ${ }^{13} \mathrm{C}$ NMR $\left(\mathrm{C}_{6} \mathrm{D}_{6}, 400 \mathrm{MHz}\right) \quad \delta 171.8,102.7,80.7,73.9,73.0,68.4,67.5,66.1,59.7$, 57.8, 51.3, 39.5, 37.7, 35.4, 34.4, 34.0, 26.1, 25.9, 18.3, 18.2, 12.0, 5.2, -4.1, -4.2, -4.5, -4.7, -5.1, -5.2; HRMS (ESI) calculated for $\mathrm{C}_{36} \mathrm{H}_{76} \mathrm{O}_{10} \mathrm{Si}_{3}(\mathrm{M}+\mathrm{Na})$ 775.4644, found 775.4655 .

\section{$\underline{\text { Preparation of carbonate } \mathbf{2 6}}$}

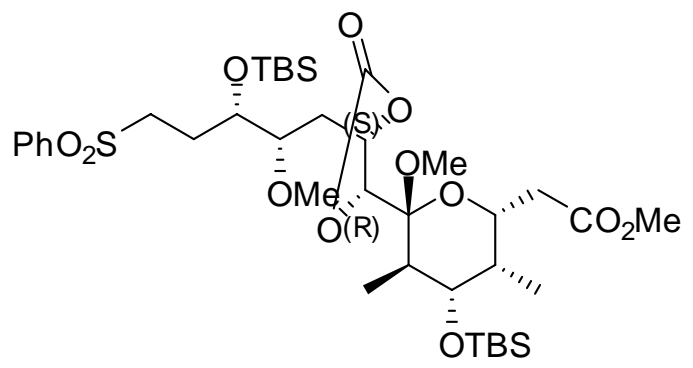

To a solution of 25 (40 mg, $0.051 \mathrm{mmol})$ and pyridine (0.25 ml, $3.09 \mathrm{mmol})$, in $\mathrm{CH}_{2} \mathrm{Cl}_{2}$ $(1.03 \mathrm{ml})$ pre-cooled to $-78{ }^{\circ} \mathrm{C}$ under an atmosphere of $\mathrm{N}_{2}$ was added a solution of triphosgene (30.5 $\mathrm{mg}, 0.10 \mathrm{mmol}$ ) in $\mathrm{CH}_{2} \mathrm{Cl}_{2}(0.2 \mathrm{ml})$ and the reaction solution reached 0 ${ }^{\circ} \mathrm{C}$ in $3 \mathrm{~h}$. After $1 \mathrm{~h}$ stirring at $0{ }^{\circ} \mathrm{C}$, the reaction was quenched with sat. $\mathrm{NH}_{4} \mathrm{Cl}$. The crude mixture was transferred to a separatory funnel and extracted with $\mathrm{CH}_{2} \mathrm{Cl}_{2}(2 \times 10$ 
$\mathrm{mL}$ ), dried with $\mathrm{Na}_{2} \mathrm{SO}_{4}$ and concentrated using a rotary evaporator to afford a fluffy yellow solid $37 \mathrm{mg}$ (95\%) of carbonate 26.

26 ; ${ }^{1} \mathrm{H}$ NMR $\left(\mathrm{C}_{6} \mathrm{D}_{6}, 300 \mathrm{MHz}\right) \quad \delta 7.89(\mathrm{~m}, 2 \mathrm{H}), 6.94(\mathrm{~m}, 3 \mathrm{H}), 4.94(\mathrm{~m}, 1 \mathrm{H}), 4.24(\mathrm{td}, J$ $=9.9 \mathrm{~Hz}, 2.4 \mathrm{~Hz}, 1 \mathrm{H}), 4.06$ (d, $J=4.5 \mathrm{~Hz}, 1 \mathrm{H}), 4.06$ (quint, $J=4.8 \mathrm{~Hz}, 1 \mathrm{H}$ ), 3.89 (dd, $J=$ $4.8 \mathrm{~Hz}, 9.9$ Hz, 1H), 3.39 (s, 3H), 3.36 (d, J=4.2 Hz, 1H), 3.14 (s, 3H), 3.13 (s, 3H), 3.10 (m, 2H), 2.47 (dd, $J=9.9 \mathrm{~Hz}, 15.6 \mathrm{~Hz}, 1 \mathrm{H}$ ), 2.22 (m, 2H), 1.89 (dd, $J=2.7 \mathrm{~Hz}, 15.3 \mathrm{~Hz}$, 1H), 1.80 (m, 3H), 1.25 (d, $J=6.6 \mathrm{~Hz}, 3 \mathrm{H}), 0.95$ (s, 9H), 0.90 (s, 9H), 0.87 (d, $J=7.2$ $\mathrm{Hz}, 3 \mathrm{H}), 0.08$ (s, 3H), 0.03 (s, 3H), 0.00 (s, 3H), -0.01 (s, 3H) ; ${ }^{13} \mathrm{C}$ NMR $\left(\mathrm{C}_{6} \mathrm{D}_{6}, 300\right.$ MHz) $\delta 171.3,153.4,140.5,133.2,129.2,100.7,80.5,79.6,74.9,73.4,69.1,68.7,58.3$, 53.0, 51.1, 48.9, 38.9, 37.4, 35.6, 34.4, 25.9, 25.8, 18.2, 18.0, 11.9, 5.2, -4.2, -4.3, -4.7 ; $\mathrm{mp}=141.0-145.0{ }^{\circ} \mathrm{C}$; the structure was confirmed via X-ray analysis (See attached cif file).

Preparation of epoxide 27

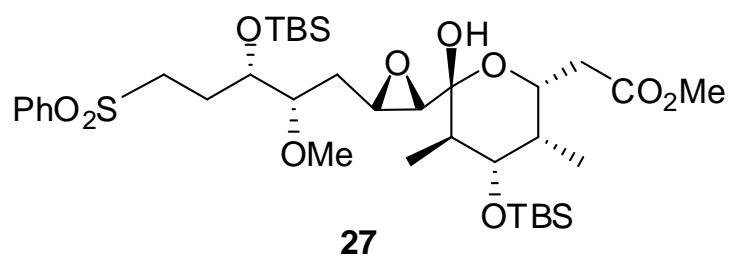

To (z)-allylic hemiacetal 8 (75 mg, $0.10 \mathrm{mmol})$ was added aq DMDO solution ( 0.1 M, in acetone, $3.09 \mathrm{ml}, 0.30 \mathrm{mmol}$ ) at rt. After $3 \mathrm{~h}$ stirring at rt, the reaction solution was concentrated to give a yellow oil. The crude oil was purified by flash column chromatography (hexanes: ethyl acetate $=7: 3$ ) to afford $57 \mathrm{mg}(75 \%)$ of major epoxide 27 and $19 \mathrm{mg}$ (25\%) of minor epoxide.

27 (major epoxide) ; ${ }^{1} \mathrm{H}$ NMR $\left(\mathrm{C}_{6} \mathrm{D}_{6}, 300 \mathrm{MHz}\right) \delta 7.90$ (m, 2H), 6.99 (3s, 3H), 4.72 (m, 1H), 4.09 (m, 1H), 4.02 (dd, $J=4.5 \mathrm{~Hz}, 10.5 \mathrm{~Hz}, 1 \mathrm{H}), 3.71$ (s, 1H), 3.47 (s, 3H), 3.29 (m, 1H), 3.23 (s, 3H), 3.19 (t, $J=6.9 \mathrm{~Hz}, 2 \mathrm{H}), 3.04$ (m, 1H), 2.86 (d, $J=4.8 \mathrm{~Hz}, 1 \mathrm{H}), 2.57$ (dd, $J=9.3$ Hz, 15.3 Hz, 1H), 2.24 (m, 2H), 2.15 (dd, $J=3.9$ Hz, 15.3 Hz, 1H), 1.95 (m, 
3H), 1.21 (d, $J=6.6 \mathrm{~Hz}, 3 \mathrm{H}), 0.98$ (d, $J=6.9 \mathrm{~Hz}, 3 \mathrm{H}), 0.98$ (s, 9H), 0.89 (s, 9H), 0.08 (s, 3H), 0.04 (s, 6H), -0.00 (s, 3H) ; ${ }^{13} \mathrm{C}$ NMR $\left(\mathrm{C}_{6} \mathrm{D}_{6}, 300 \mathrm{MHz}\right) \delta 171.5,140.7,133.5$, 129.5, 97.8, 82.6, 73.6, 70.6, 69.1, 61.4, 58.8, 56.1, 53.2, 51.7, 41.8, 39.5, 38.5, 26.4, 26.3, 18.6, 18.5, 12.6, 5.7, -3.8, -3.9, -4.2, -4.4. ; LRMS (ESI) calculated for $\mathrm{C}_{36} \mathrm{H}_{64} \mathrm{O}_{10} \mathrm{SSi}_{2}(\mathrm{M}+\mathrm{Na})$ 767.37, found 767.29.

Minor epoxide; ${ }^{1} \mathrm{H}$ NMR $\left(\mathrm{C}_{6} \mathrm{D}_{6}, 300 \mathrm{MHz}\right) \delta 7.88(\mathrm{~m}, 2 \mathrm{H}), 7.00(\mathrm{~m}, 3 \mathrm{H}), 4.76(\mathrm{~m}, 1 \mathrm{H})$, 4.09 (m, 2H), 3.70 (s, 1H), 3.34 (s, 3H), 3.27 (m, 1H), 3.17 (s, 3H), 3.14 (m, 2H), 3.96 (m, 1H), 2.80 (d, $J=4.2 \mathrm{~Hz}, 1 \mathrm{H}), 2.70$ (dd, $J=8.7 \mathrm{~Hz}, 15.9 \mathrm{~Hz}, 1 \mathrm{H}), 2.21$ (dd, $J=4.8$ Hz, 15.9 Hz, 1H), 2.10 (m, 3H), 1.88 (m, 3H), 1.03 (d, $J=~ 8.7$ Hz, 6H), 1.01 (s, 9H), 0.88 (s, 9H), 0.08 (s, 6H), 0.03 (s, 3H), 0.00 (s, 3H) ; ${ }^{13} \mathrm{C}$ NMR $\left(\mathrm{C}_{6} \mathrm{D}_{6}, 300 \mathrm{MHz}\right) \delta$ 172.0, 140.5, 133.5, 129.5, 98.6, 83.6, 73.4, 71.8, 67.9, 60.2, 58.0, 55.0, 53.1, 51.6, 39.6, 38.1, 38.0, 26.4, 18.7, 18.6, 12.9, 5.7, -3.7, -3.8, -4.3, -4.4. ; LRMS (ESI) calculated for $\mathrm{C}_{36} \mathrm{H}_{64} \mathrm{O}_{10} \mathrm{SSi}_{2}(\mathrm{M}+\mathrm{Na})$ 767.37, found 767.25.

\section{Preparation of dioxolane $\mathbf{2 9}$}

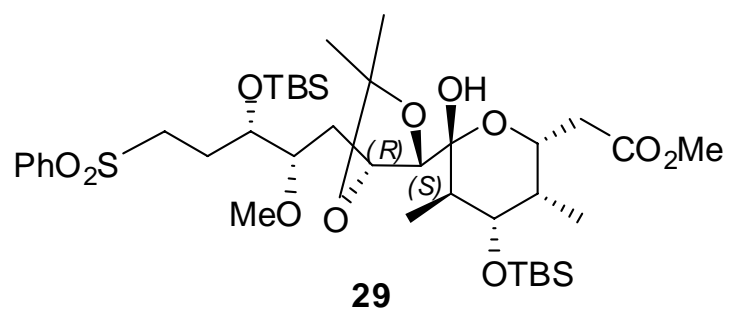

To a stirred solution of 27 (30 $\mathrm{mg}, 0.040 \mathrm{mmol})$ in distilled acetone $(0.40 \mathrm{~mL})$ was added anhydrous $\mathrm{SnCl}_{2}(1.52 \mathrm{mg}, 0.008 \mathrm{mmol})$. The resulting solution was heated to $50 \sim 55{ }^{\circ} \mathrm{C}$ and stirred for 20 min. The reaction was quenched with sat. $\mathrm{NaHCO}_{3}$. The crude mixture was transferred to a separatory funnel, extracted with ethyl acetate $(2 \times 5 \mathrm{~mL})$ and dried with $\mathrm{Na}_{2} \mathrm{SO}_{4}$. The crude oil concentrated was purified by flash column chromatography (hexanes: ethyl acetate $=7: 3)$ to afford 25 mg (80 \%) of dioxolane 29. 
29 ; ${ }^{1} \mathrm{H}$ NMR $\left(\mathrm{C}_{6} \mathrm{D}_{6}, 300 \mathrm{MHz}\right) \quad \delta 7.88(\mathrm{~m}, 2 \mathrm{H}), 6.96(\mathrm{~m}, 3 \mathrm{H}), 4.82(\mathrm{~m}, 1 \mathrm{H}), 4.58(\mathrm{~m}$, 1H), 4.10 (m, 1H), 4.07 (d, J = 6.9 Hz, 2H), 3.36 (s, 3H), 3.25 (m, 3H), 3.15 (s, 3H), 2.60 (dd, $J=8.7 \mathrm{~Hz}, 15.3 \mathrm{~Hz}, 1 \mathrm{H}), 2.18$ (m, 2H), 2.13 (dd, $J=3.9 \mathrm{~Hz}, 15.0 \mathrm{~Hz}, 1 \mathrm{H}$ ), 1.91 (m, 4H), 1.43 (d, $J=9.3 \mathrm{~Hz}, 6 \mathrm{H}), 1.23$ (d, $J=6.3 \mathrm{~Hz}, 3 \mathrm{H}$ ), 0.97 (d, $J=7.0 \mathrm{~Hz}, 3 \mathrm{H}$ ), 0.95 (s, 9H), 0.90 (s, 9H), 0.10 (s, 3H), 0.04 (s, 6H), -0.01 (s, 3H) ; ${ }^{13} \mathrm{C}$ NMR $\left(\mathrm{C}_{6} \mathrm{D}_{6}, 300 \mathrm{MHz}\right)$ $\delta 171.2,140.3,133.0,129.1,109.4,98.0,83.3,80.9,73.7,73.5,70.7,67.7,57.2,52.6$, 51.0, 39.2, 37.6, 37.2, 33.1, 28.2, 26.7, 26.1, 26.0, 18.2, 12.4, 5.4, -4.1, -4.7, -4.8 ; LRMS (ESI) calculated for $\mathrm{C}_{39} \mathrm{H}_{70} \mathrm{O}_{11} \mathrm{SSi}_{2}(\mathrm{M}+\mathrm{Na})$ 825.41, found 825.27.

\section{Preparation of iodide $\mathbf{3 0}$}

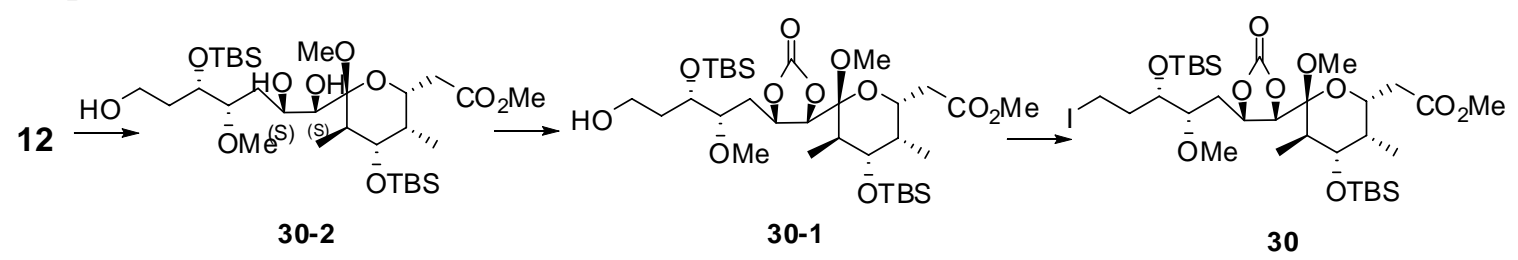

To a stirred solution of 12 (66 mg, $0.087 \mathrm{mmol})$ in $\mathrm{MeOH}(0.87 \mathrm{~mL})$ were added PPTS (pyridinium p-Toluenesulfonate, $6.5 \mathrm{mg}, 0.026 \mathrm{mmol}$ ) at rt. After $10 \mathrm{~h}$ stirring at rt, the reaction was quenched with triethylamine. The mixture concentrated was purified by flash column chromatography (hexanes: ethyl acetate $=6: 4$ ) to afford $42 \mathrm{mg}$ (75 \%) of primary desilylated methoxy acetal 30-2. To a solution of 30-2 (42 mg, $0.064 \mathrm{mmol}$ ) and pyridine $(0.31 \mathrm{ml}, 3.84 \mathrm{mmol})$, in $\mathrm{CH}_{2} \mathrm{Cl}_{2}(1.28 \mathrm{ml})$ pre-cooled to $-78{ }^{\circ} \mathrm{C}$ under an atmosphere of $\mathrm{N}_{2}$ was added a solution of triphosgene (39.0 mg, $0.12 \mathrm{mmol}$ ) in $\mathrm{CH}_{2} \mathrm{Cl}_{2}$ $(0.25 \mathrm{ml})$ and the reaction solution reached $0{ }^{\circ} \mathrm{C}$ over $3 \mathrm{~h}$. After $1 \mathrm{~h}$ stirring at $0{ }^{\circ} \mathrm{C}$, the reaction was quenched with sat. $\mathrm{NH}_{4} \mathrm{Cl}$. The crude mixture was transferred to a separatory funnel and extracted with $\mathrm{CH}_{2} \mathrm{Cl}_{2}(2 \times 10 \mathrm{~mL})$, dried with $\mathrm{Na}_{2} \mathrm{SO}_{4}$ and the mixture concentrated and purified by flash column chromatography (hexanes: ethyl acetate $=7: 3)$ to afford oil $38 \mathrm{mg}(85 \%)$ of carbonate $\mathbf{3 0 - 1}$. To a solution of $\mathbf{3 0 - 1}$ (38 $\mathrm{mg}, 0.056 \mathrm{mmol})$ and imidazole (19 $\mathrm{mg}, 0.28 \mathrm{mmol})$, in THF (1.12 ml) pre-cooled to 0 
${ }^{\circ} \mathrm{C}$ under an atmosphere of $\mathrm{N}_{2}$ was added triphenyl phosphine (22.0 mg, $0.084 \mathrm{mmol}$ ) followed by Iodine $(21.3 \mathrm{mg}, 0.084 \mathrm{mmol})$. After $1 \mathrm{~h}$ stirring at $0{ }^{\circ} \mathrm{C}$, the reaction was quenched with sat $\mathrm{NaHCO}_{3}$ followed by washing with sat $\mathrm{Na}_{2} \mathrm{~S}_{2} \mathrm{O}_{3}$ until the organic solution became colorless. The crude mixture was transferred to a separatory funnel and extracted with ethyl acetate $(2 \times 10 \mathrm{~mL})$, dried with $\mathrm{Na}_{2} \mathrm{SO}_{4}$ and concentrated using a rotary evaporator to afford yellow oil $39 \mathrm{mg}$ (90 \%) of iodide $3 \mathbf{3 0 .}$

30: ${ }^{1} \mathrm{H}$ NMR $\left(\mathrm{C}_{6} \mathrm{D}_{6}, 400 \mathrm{MHz}\right) \quad \delta 4.58$ (m, 1H), $4.32(\mathrm{~m}, 1 \mathrm{H}), 4.14$ (d, J=6.6 Hz, 1H), 4.04 (m, 1H), 3.98 (dd, $J=4.9$ Hz, $10.0 \mathrm{~Hz}, 1 \mathrm{H}$ ), 3.52 (m, 1H), 3.45 (s, 3H), 3.44 (s, 3H), 3.16 (s, 3H), 3.07 (m, 1H), 3.00 (m, 1H), 2.55 (dd, $J=9.5 \mathrm{~Hz}, 15.3 \mathrm{~Hz}, 1 \mathrm{H}), 2.41$ (t, $J=13.0 \mathrm{~Hz}, 1 \mathrm{H}$ ), 2.04 (dd, $J=3.9 \mathrm{~Hz}, 15.4 \mathrm{~Hz}, 1 \mathrm{H}), 2.03$ (m, 1H), 1.81 (m, 1H), 1.73 (m, 2H), 1.61 (m, 1H), 0.97 (s, 9H), 0.96 (d, $J=6.6 \mathrm{~Hz}, 3 \mathrm{H}), 0.91$ (s, 9H), 0.89 (d, $J=6.7$ Hz, 3H), 0.20 (s, 3H), 0.10 (s, 3H), 0.00 (s, 3H), -0.03 (s, 3H) ; ${ }^{13} \mathrm{C}$ NMR $\left(\mathrm{C}_{6} \mathrm{D}_{6}, 400\right.$ MHz) $\delta 171.0,153.4,99.5,81.7,79.0,76.0,72.3,70.0,69.2,57.8,51.5,51.4,38.7,37.6$, 37.4, 35.2, 29.6, 25.9, 18.2, 18.0, 11.7, 6.5, 4.2, -4.1, -4.2, -4.4, -4.7 ; HRMS (ESI) calculated for $\mathrm{C}_{32} \mathrm{H}_{61} \mathrm{IO}_{10} \mathrm{Si}_{2}(\mathrm{M}+\mathrm{Na})$ 811.2746, found 811.2759. 
Table 3. ${ }^{13} \mathrm{C}$ NMR data

\begin{tabular}{|c|c|c|c|c|c|c|c|c|c|c|c|c|c|}
\hline & \multicolumn{8}{|c|}{ Hemiacetal } & \multicolumn{2}{|c|}{ Methoxy acetal } & \multicolumn{2}{|c|}{ Epoxy hemiacetal } & \multirow{2}{*}{$\begin{array}{c}\text { dioxolane } \\
19\end{array}$} \\
\hline & $\begin{array}{c}11 \\
(19 \mathrm{~S}, 20 \mathrm{~S})\end{array}$ & $\begin{array}{c}14 \\
(19 R, 20 R)\end{array}$ & $\begin{array}{c}12 \\
(195,20 s)\end{array}$ & $\begin{array}{c}15 \\
(19 R, 20 R)\end{array}$ & $\begin{array}{c}20 \\
(19 R, 20 S)\end{array}$ & $\begin{array}{c}23 \\
(19 \mathrm{~S}, 20 \mathrm{R})\end{array}$ & $\begin{array}{c}21 \\
(19 R, 20 s)\end{array}$ & $\begin{array}{c}24 \\
(19 \mathrm{~S}, 20 \mathrm{R})\end{array}$ & $\begin{array}{c}22 \\
(19 R, 20 \mathrm{~S})\end{array}$ & $\begin{array}{c}25 \\
(19 \mathrm{~S}, 20 \mathrm{R})\end{array}$ & $\begin{array}{c}27 \\
\text { (major) }\end{array}$ & $\begin{array}{c}27 \\
\text { (minor) }\end{array}$ & \\
\hline & 172.2 & 171.7 & 171.5 & 171.4 & 171.3 & 172.2 & 171.2 & 171.2 & 171.4 & 171.6 & 171.5 & 172 & 171.2 \\
\hline & 140.2 & 140.3 & & & 140.3 & 140.3 & & & 140.3 & 140.4 & 140.7 & 140.5 & 140.3 \\
\hline & 133.2 & 133.2 & & & 133.1 & 133.1 & & & 133.1 & 133 & 133.5 & 133.5 & 133 \\
\hline & 129.1 & 129.2 & & & 129.1 & 129.1 & & & 129.1 & 129.1 & 129.5 & 129.5 & 129.1 \\
\hline & 128.5 & 128.2 & & & 128.3 & 128.3 & & & 128.3 & 128.3 & 129.5 & 128.7 & 129.1 \\
\hline $\mathrm{C} 21$ & 100.3 & 101.8 & 100.8 & 101.9 & 101.1 & 102.9 & 101.2 & 102.7 & 102.1 & 103.6 & 97.8 & 98.6 & 109.4 \\
\hline \multirow[t]{25}{*}{ C17 } & 81.1 & 84.7 & 81.5 & 85.5 & 81.4 & 80.4 & 81.5 & 80.7 & 82.5 & 80.5 & 82.6 & 83.6 & 98 \\
\hline & 76.9 & 74 & 75.5 & 74.7 & 73.3 & 73.9 & 73.4 & 73.9 & 75.8 & 74.9 & & & 83.3 \\
\hline & 73.4 & 73.3 & 73.4 & 73.3 & 73.3 & 73 & 73.2 & 73 & 73.7 & 73.4 & 73.6 & 73.4 & 80.9 \\
\hline & 70.6 & 73 & 70.4 & 73 & 70.2 & 70.3 & 67.8 & 68.4 & 69.7 & 70 & 70.6 & 71.8 & 73.7 \\
\hline & 69.6 & 69.5 & 67.8 & 67.3 & 67.8 & 68.5 & 67.8 & 67.5 & 69.7 & 69 & 69.1 & 67.9 & 73.5 \\
\hline & 67.6 & 67.2 & 67.4 & 66.8 & 67.3 & 65.5 & 67.5 & 66.1 & 68.9 & 67.4 & 61.4 & 60.2 & 70.7 \\
\hline & 58.6 & 57.4 & 59.5 & 59.2 & 57.4 & 58.6 & 59.2 & 59.7 & 57.1 & 58.3 & 58.8 & 58 & 67.7 \\
\hline & 52.6 & 52.4 & 57.6 & 56.8 & 52.7 & 52.9 & 57 & 57.8 & 52.6 & 53 & 56.1 & 55 & 57.2 \\
\hline & 51.6 & 51 & 51.2 & 51 & 51.3 & 51.4 & 51.2 & 51.3 & 51.1 & $51.1 / 48.6$ & $53.2 / 51.7$ & $53.1 / 51.6$ & 52.6 \\
\hline & 39.7 & 39.3 & 39.5 & 39.3 & 39.3 & 39.5 & 39.3 & 39.5 & 39.3 & 39.1 & 41.8 & 39.6 & 51 \\
\hline & 37.8 & 37.7 & 37.8 & 37.9 & 37.7 & 37.8 & 37.8 & 37.7 & 37.8 & 37.9 & 39.5 & 38.1 & 39.2 \\
\hline & 37.4 & 36.1 & 37.6 & 36.2 & 37 & 35.3 & 37.1 & 35.4 & 37.3 & 36.5 & 38.5 & 38 & 37.6 \\
\hline & 32.9 & 32.8 & 34.6 & 34.2 & 32.8 & 33.9 & 34.6 & 34.4 & 32.6 & 34.6 & & & 37.2 \\
\hline & & & 33.4 & 32.9 & & & 32.8 & 34 & & & & & 33.1 \\
\hline & 26 & 26 & 26.1 & 26 & 26 & 25.9 & 26.1 & 26.1 & 26 & 26 & 26.4 & 26.4 & 28.2 \\
\hline & 26 & 25.8 & 26 & 25.9 & 26 & & 26 & 25.9 & & & 26.3 & & 26.7 \\
\hline & 18.2 & 18.3 & 18.3 & 18.3 & 18.2 & 18.2 & 18.3 & 18.3 & 18.2 & 18.2 & 18.6 & 18.6 & 26.1 \\
\hline & 18.1 & 18 & 18.3 & 18.2 & 18.1 & 18.1 & 18.2 & 18.2 & 18.1 & 18.1 & 18.5 & 18.6 & 26 \\
\hline & 12.3 & 12.2 & 12.4 & 12.2 & 12.5 & 12.1 & 12.5 & 12 & 12.3 & 12.4 & 12.6 & 12.9 & 18.2 \\
\hline & 5.9 & 5.3 & 5.9 & 5.3 & 5.3 & 5.2 & 5.3 & 5.2 & 5.6 & 5.6 & 5.7 & 5.7 & 12.4 \\
\hline & -4.1 & -4.1 & -4.2 & -4.1 & -4.2 & -4.1 & -4.1 & -4.1 & -4.1 & -4.1 & -3.8 & -3.7 & 5.4 \\
\hline & -4.3 & -4.4 & -4.6 & -4.2 & -4.3 & -4.2 & -4.5 & -4.2 & -4.4 & -4.3 & -3.9 & -3.8 & -4.1 \\
\hline & -4.6 & -4.6 & -4.7 & -4.6 & -4.5 & -4.6 & -4.8 & -4.5 & -4.6 & -4.6 & -4.2 & -4.3 & -4.7 \\
\hline & -4.7 & -4.7 & -5.1 & -4.7 & -4.8 & -4.7 & -5.1 & -4.7 & -4.7 & & -4.4 & -4.4 & -4.8 \\
\hline & & & -5.2 & -5.2 & & & -5.2 & $\begin{array}{l}-5.1 \\
-5.2\end{array}$ & & & & & \\
\hline
\end{tabular}




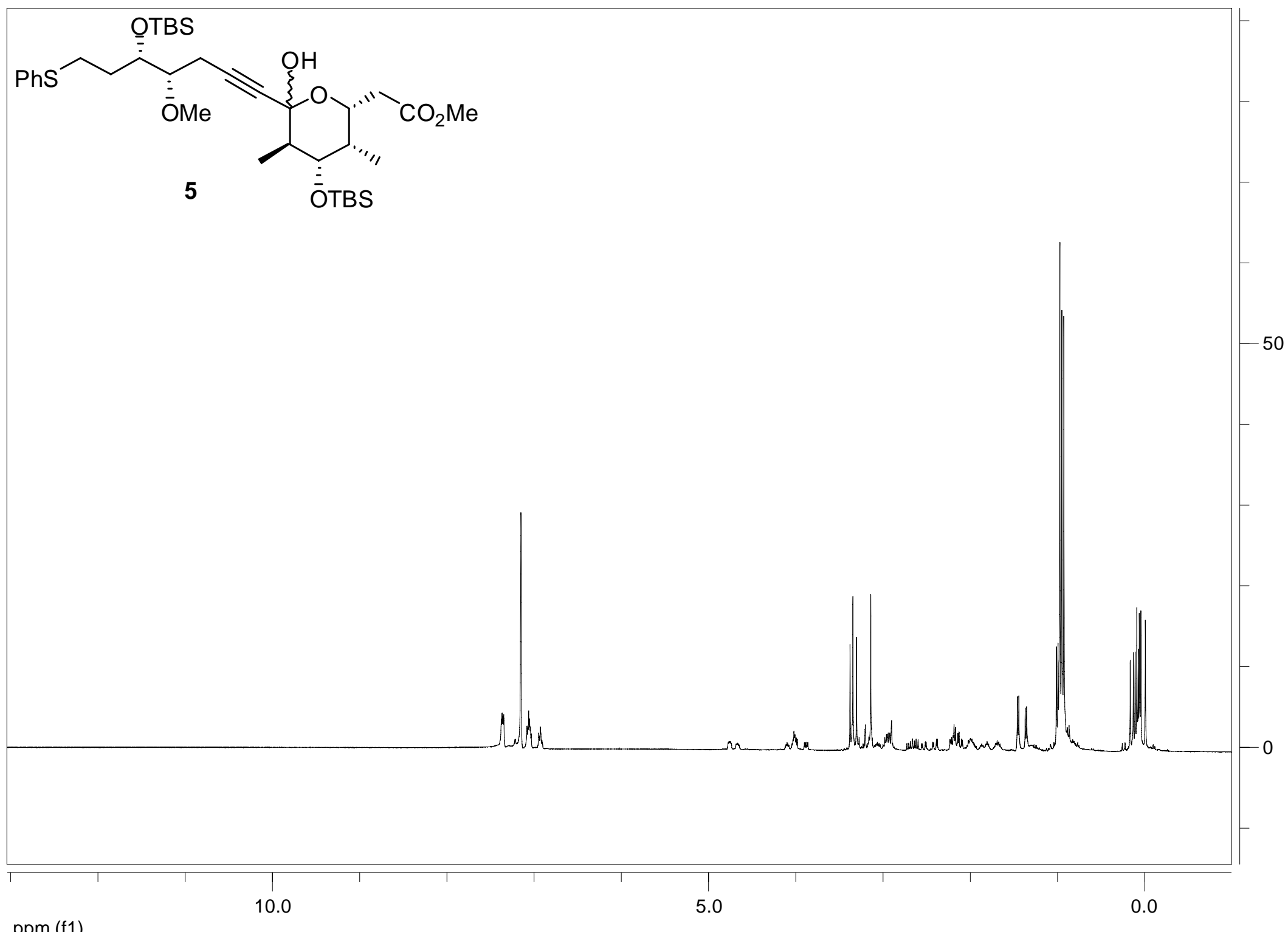

ppm (f1) 


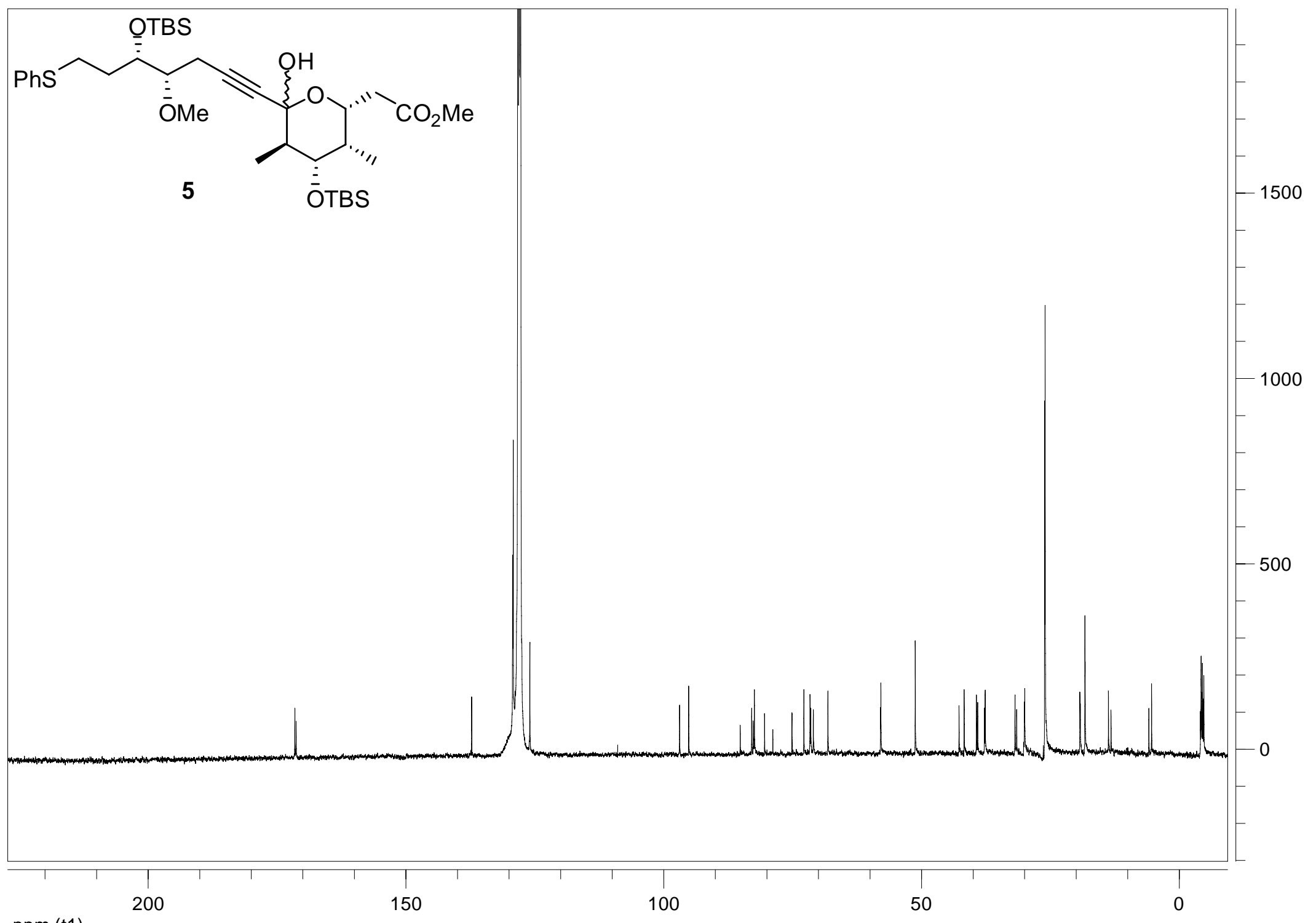

ppm (t1) 


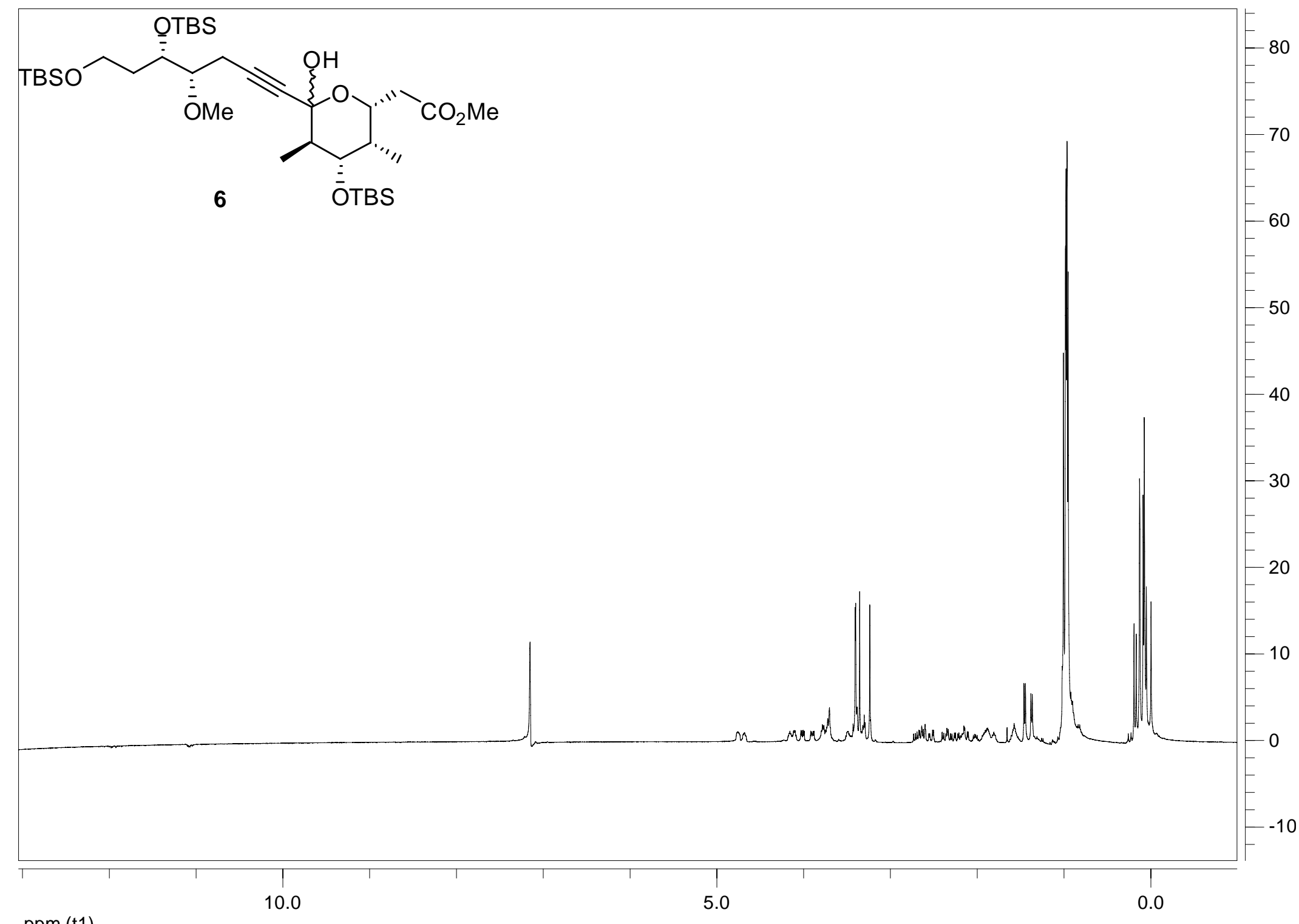

ppm (t1) 


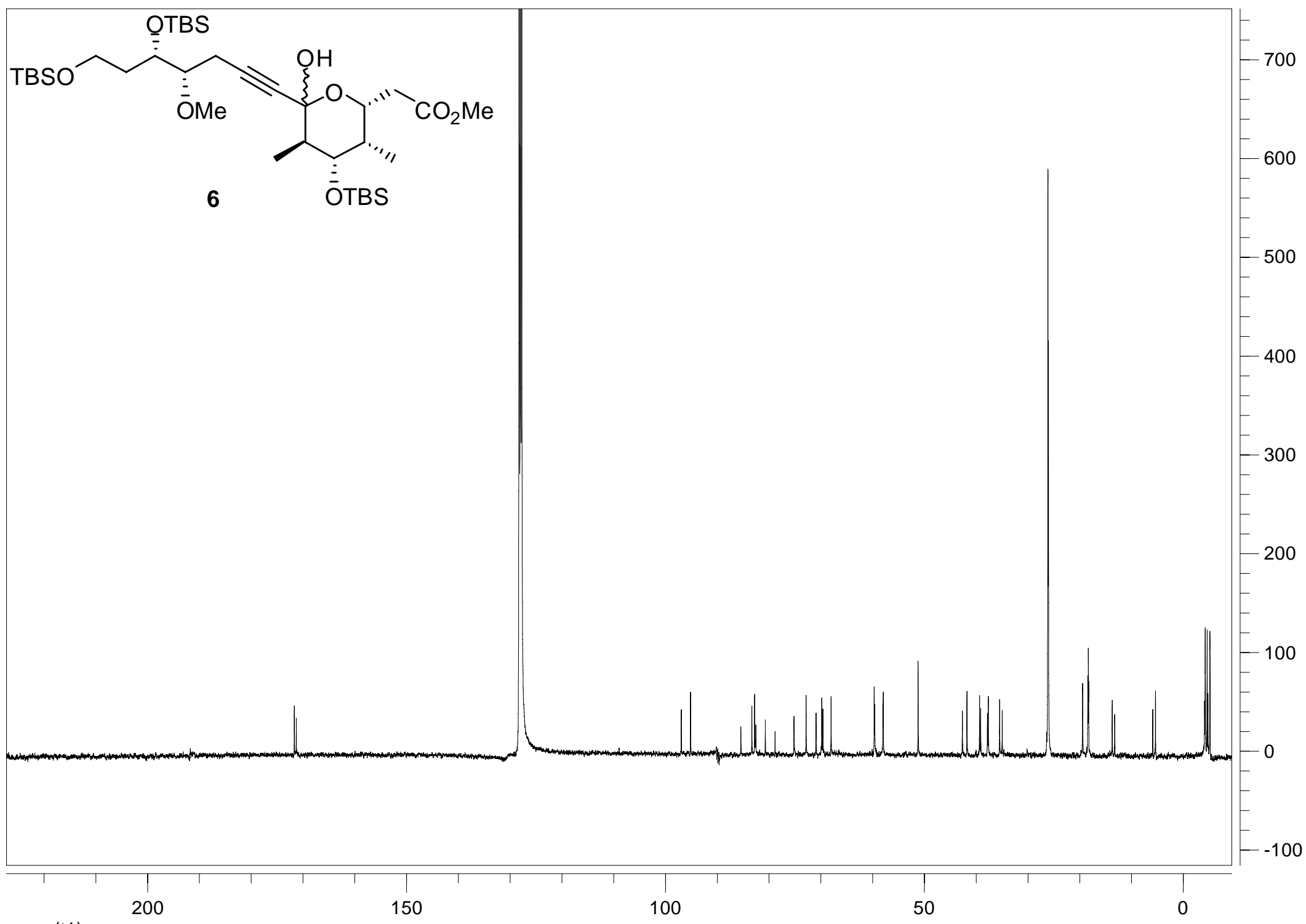

ppm (t1) 


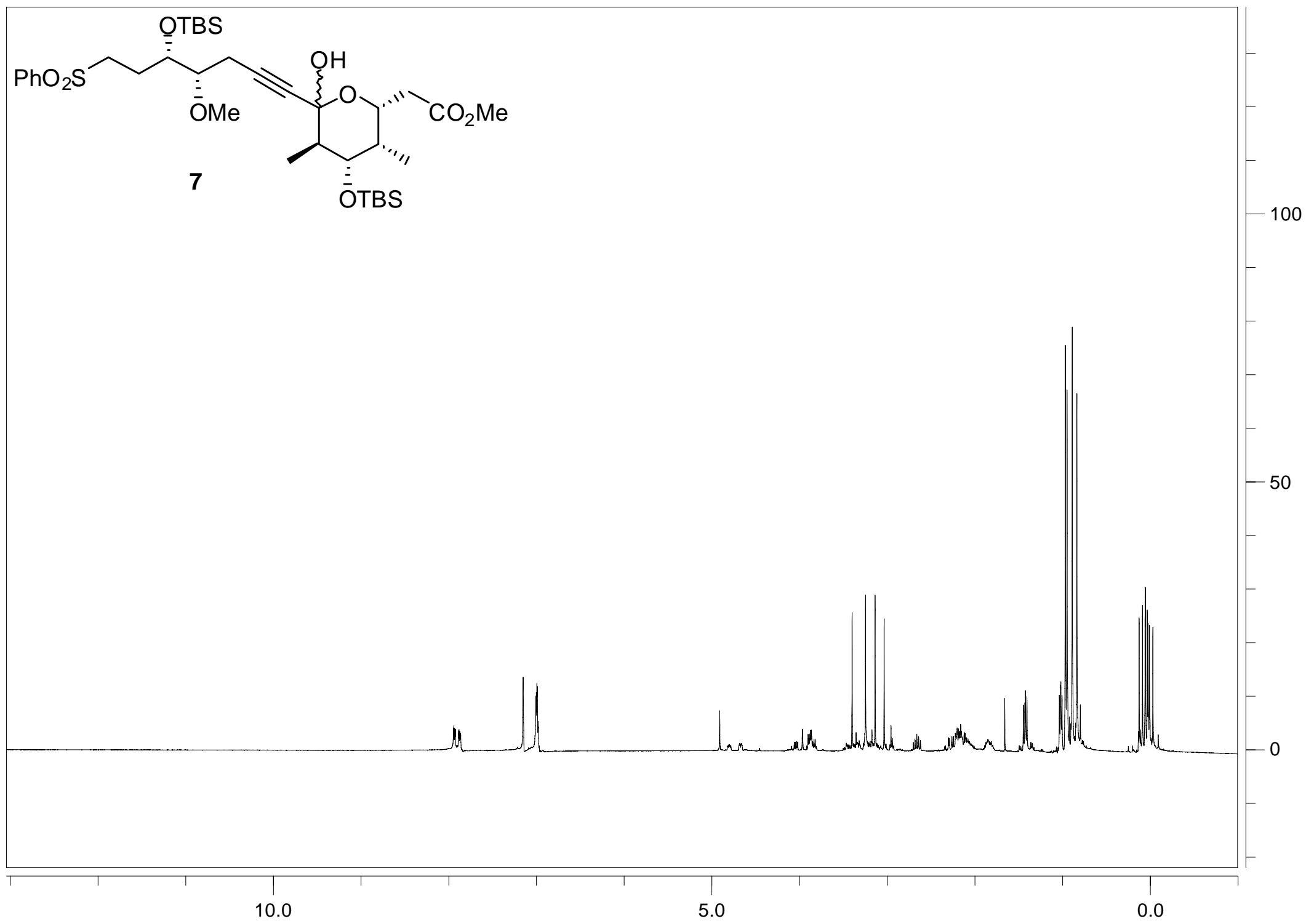

ppm (t1) 


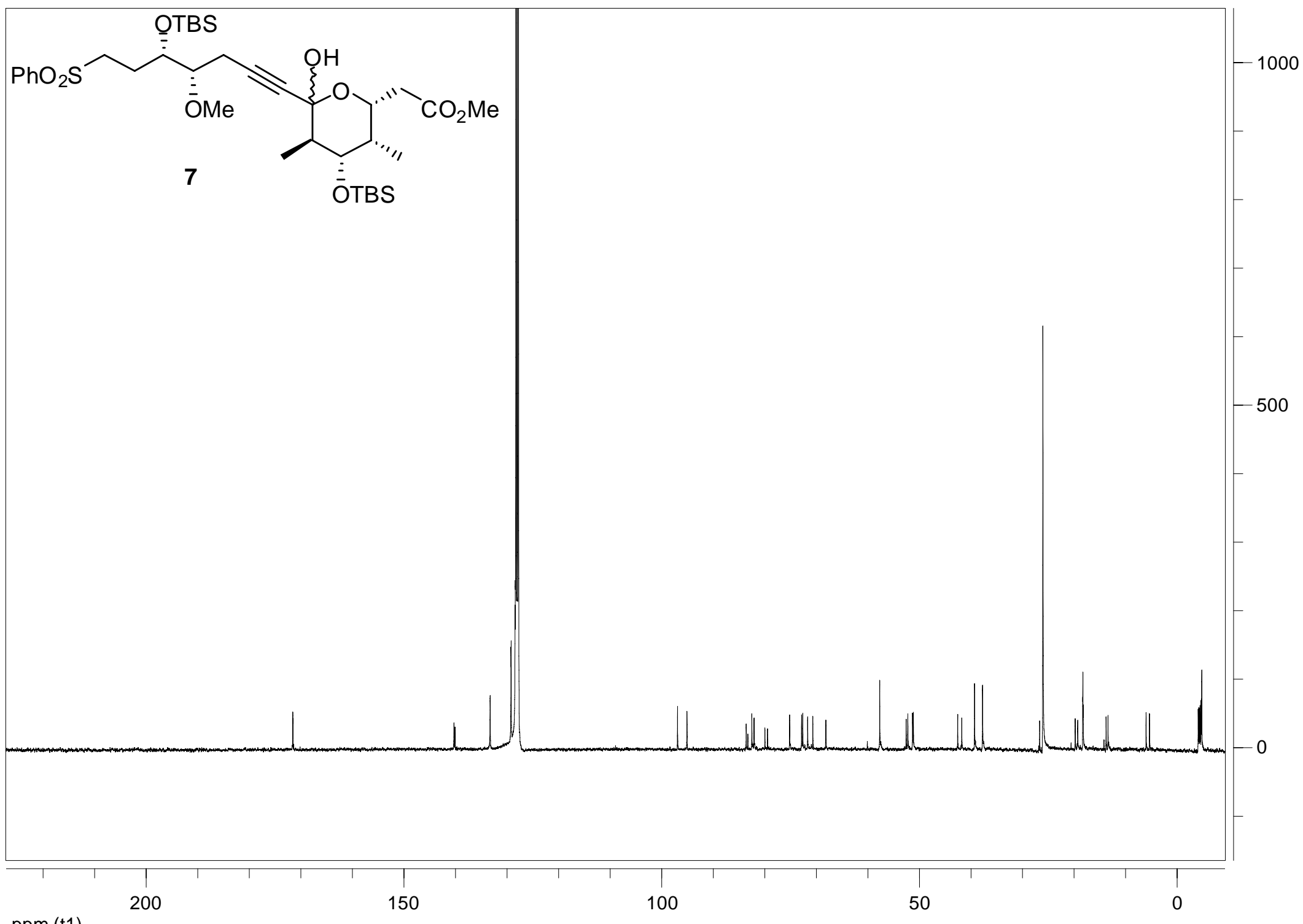

ppm (t1) 


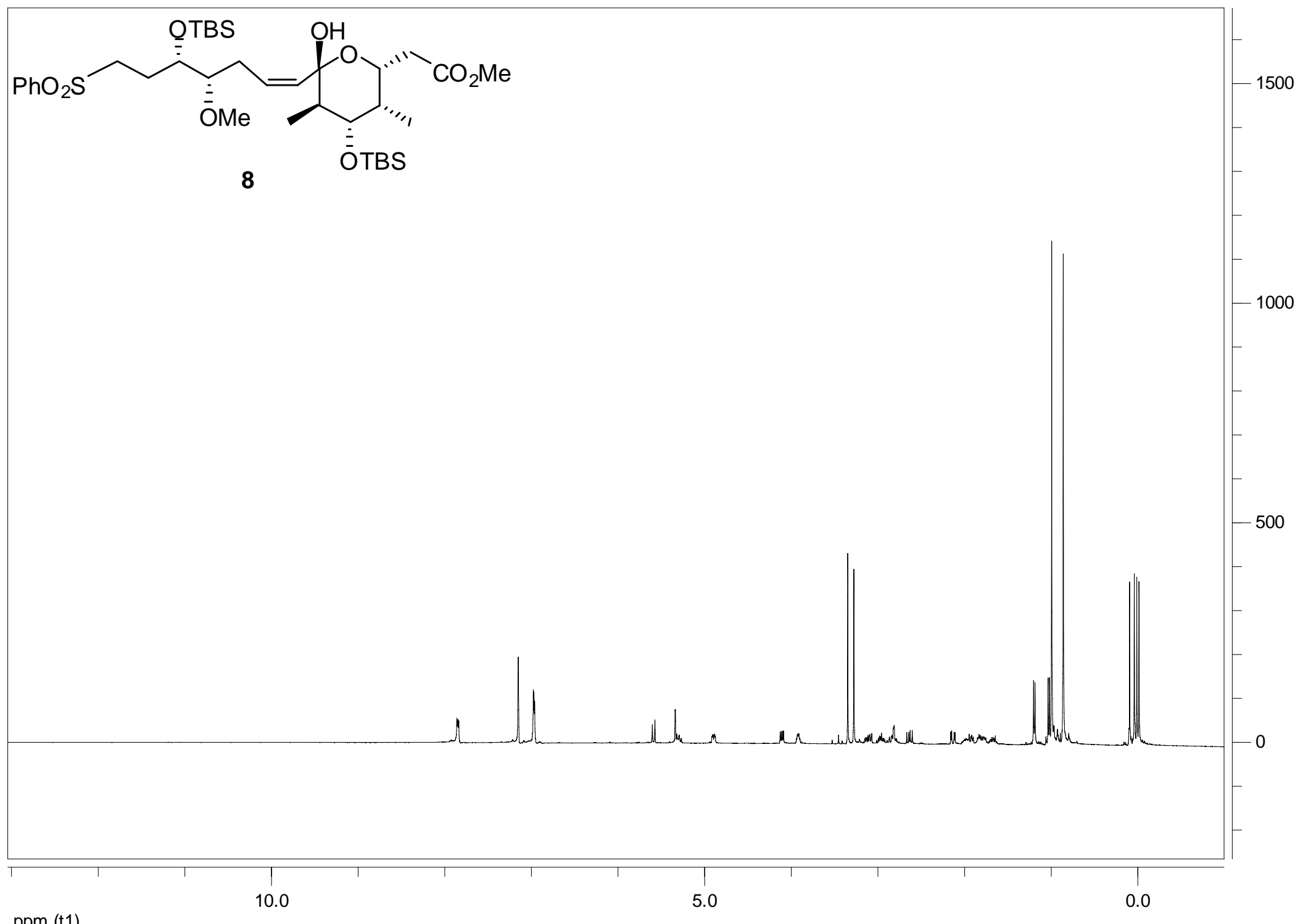

ppm (t1) 


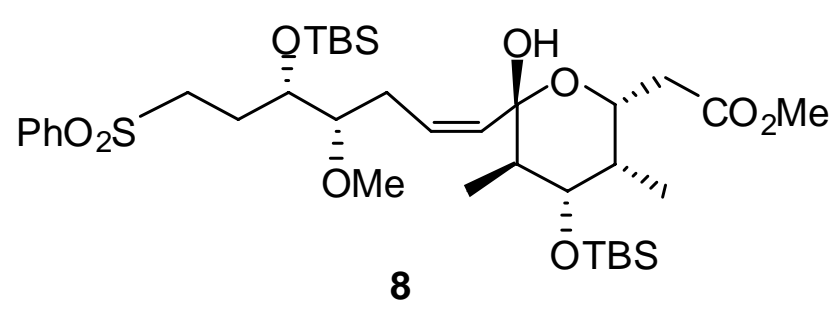

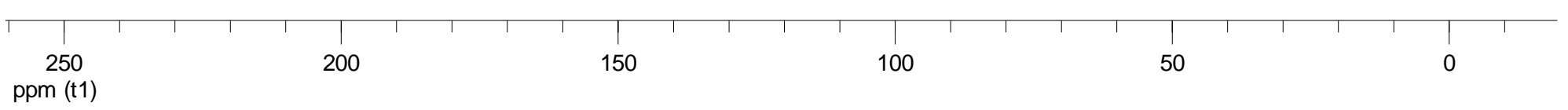




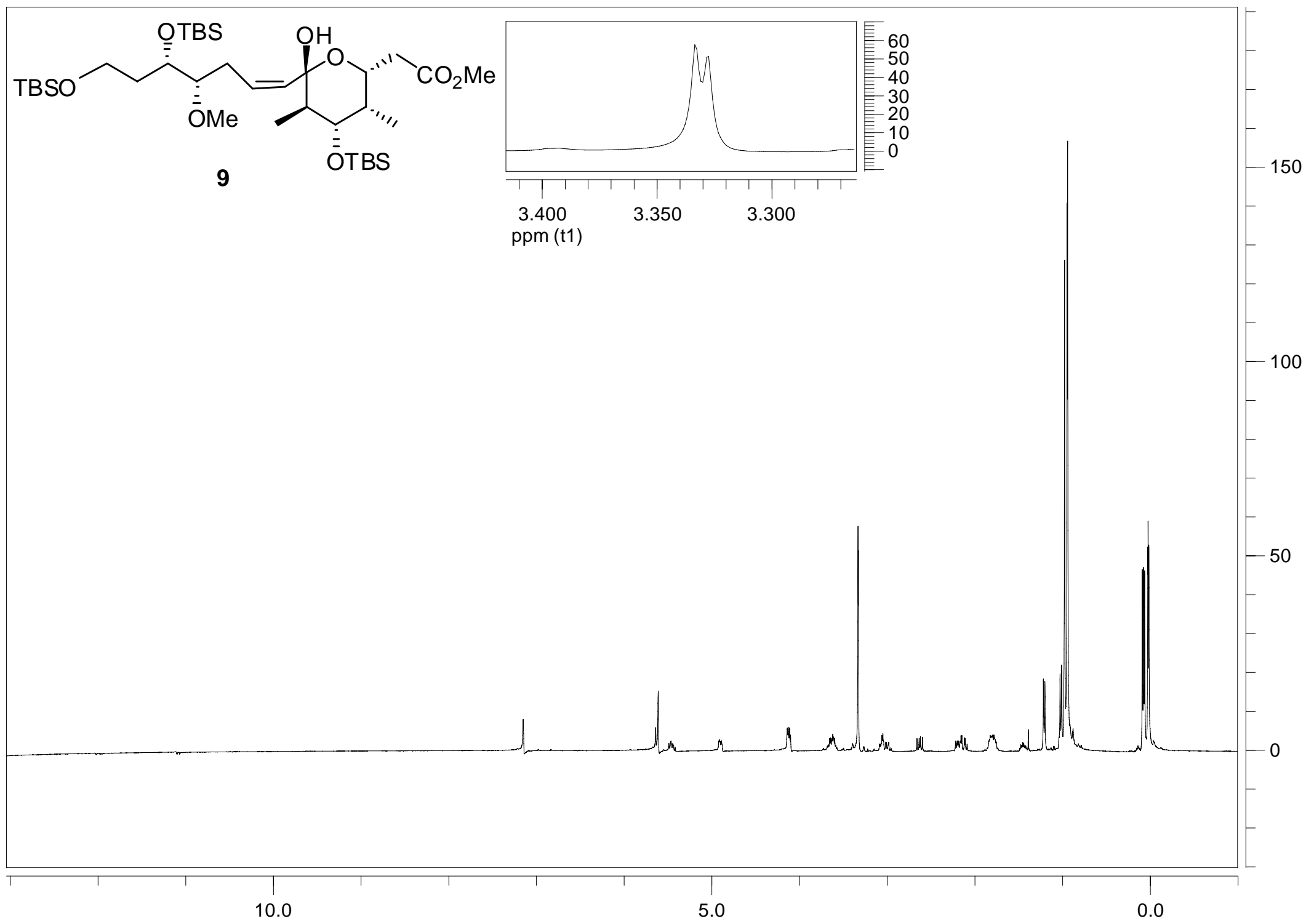

ppm (t1) 


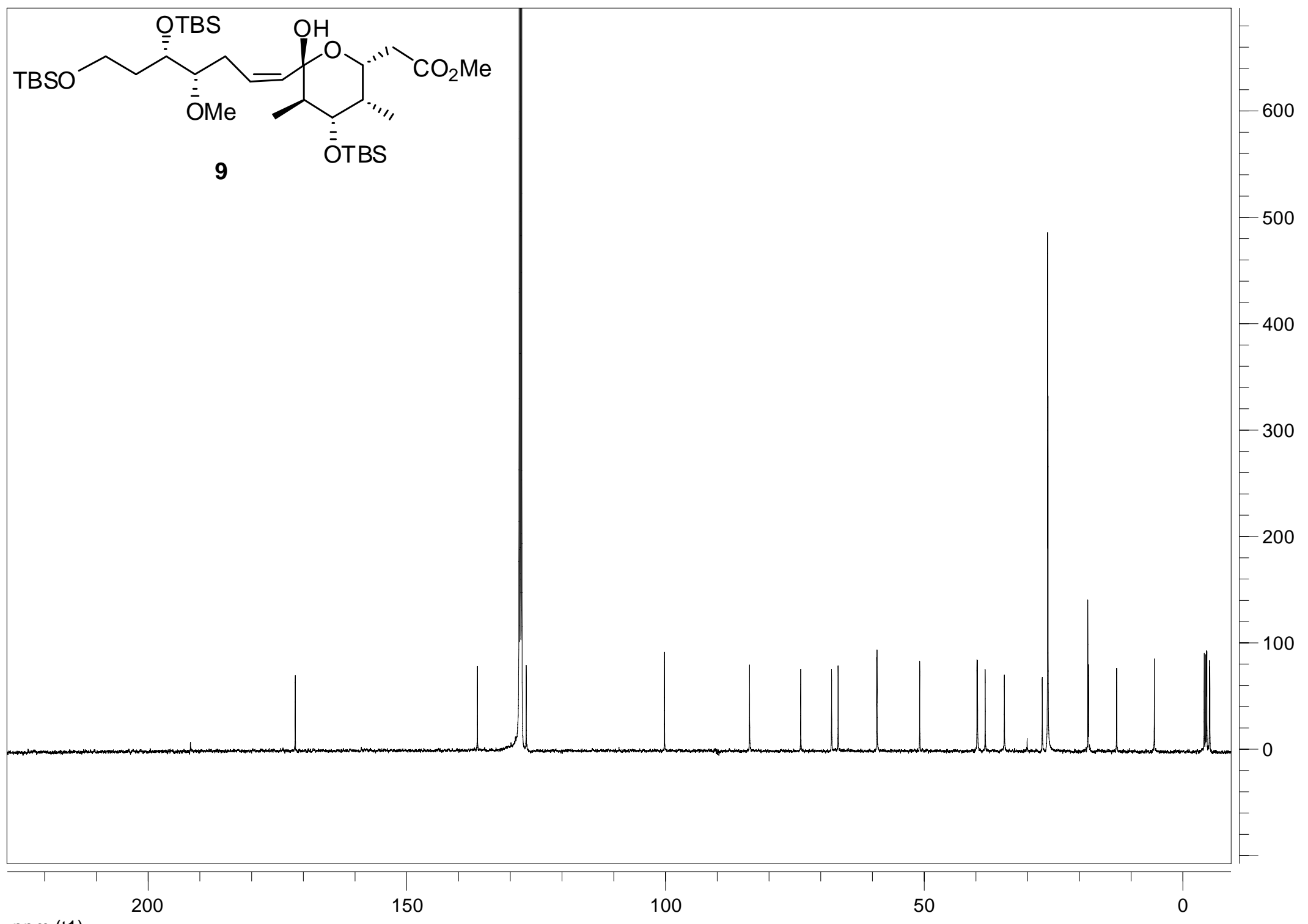

ppm (t1) 

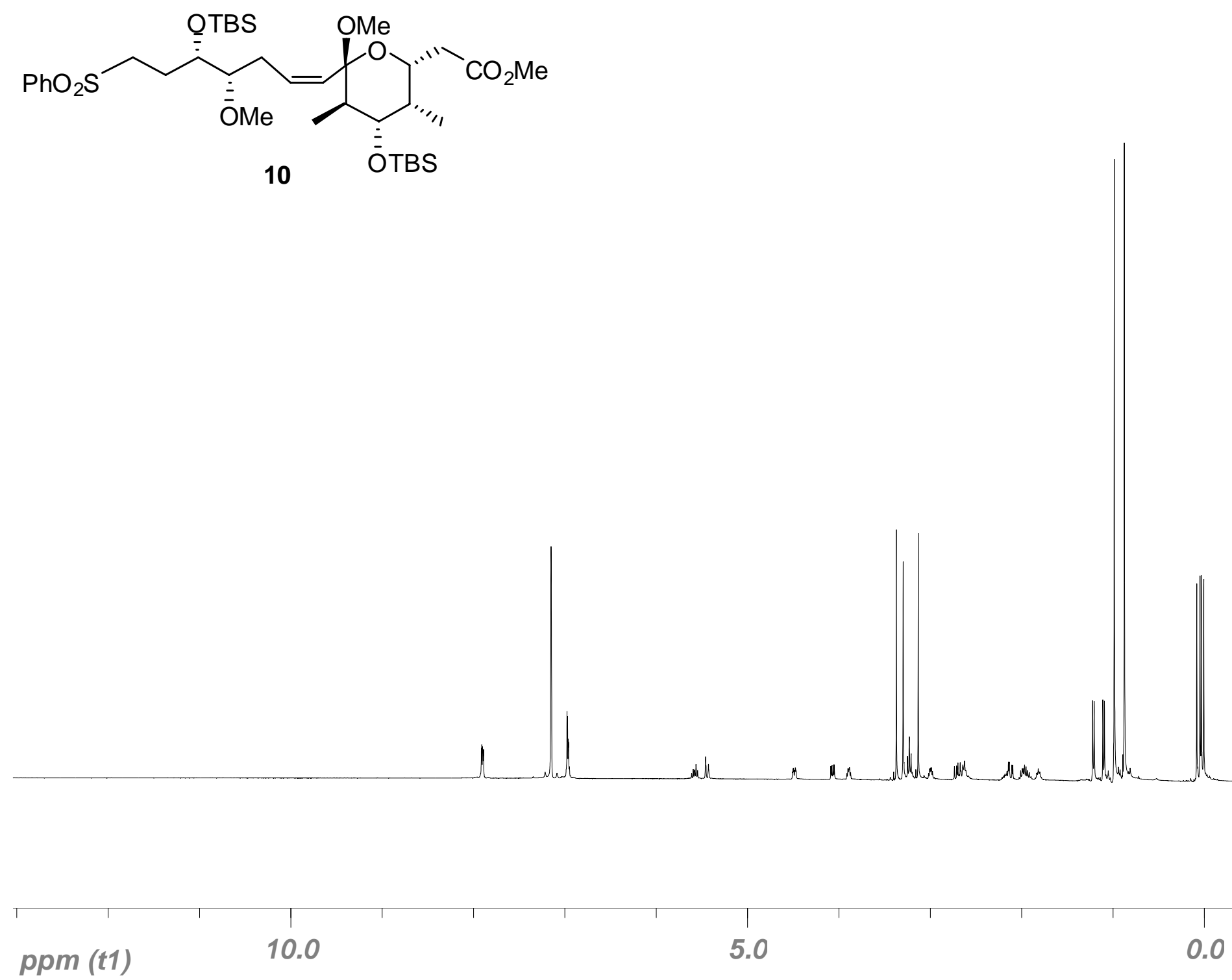


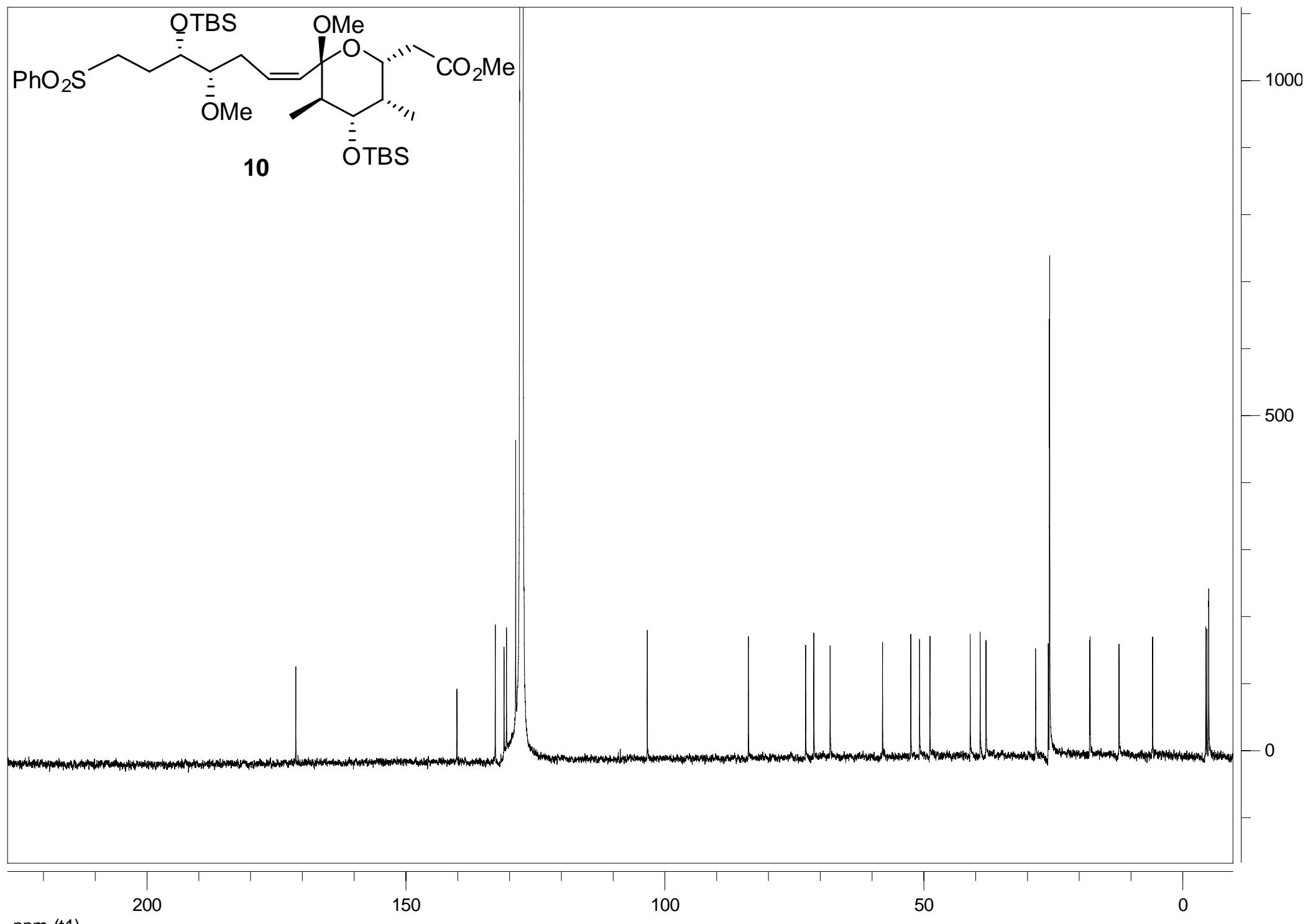

ppm (t1) 


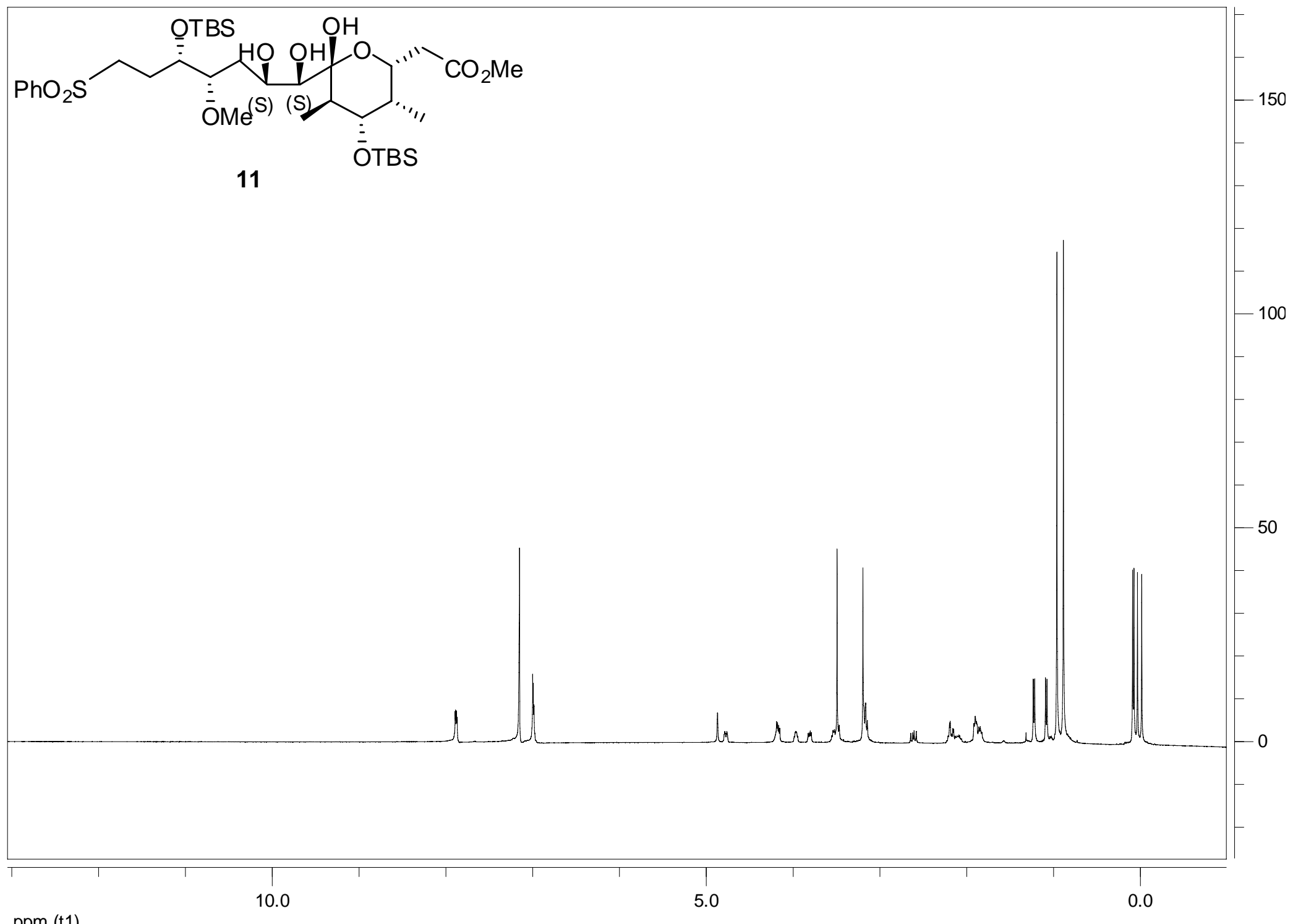

ppm (t1) 


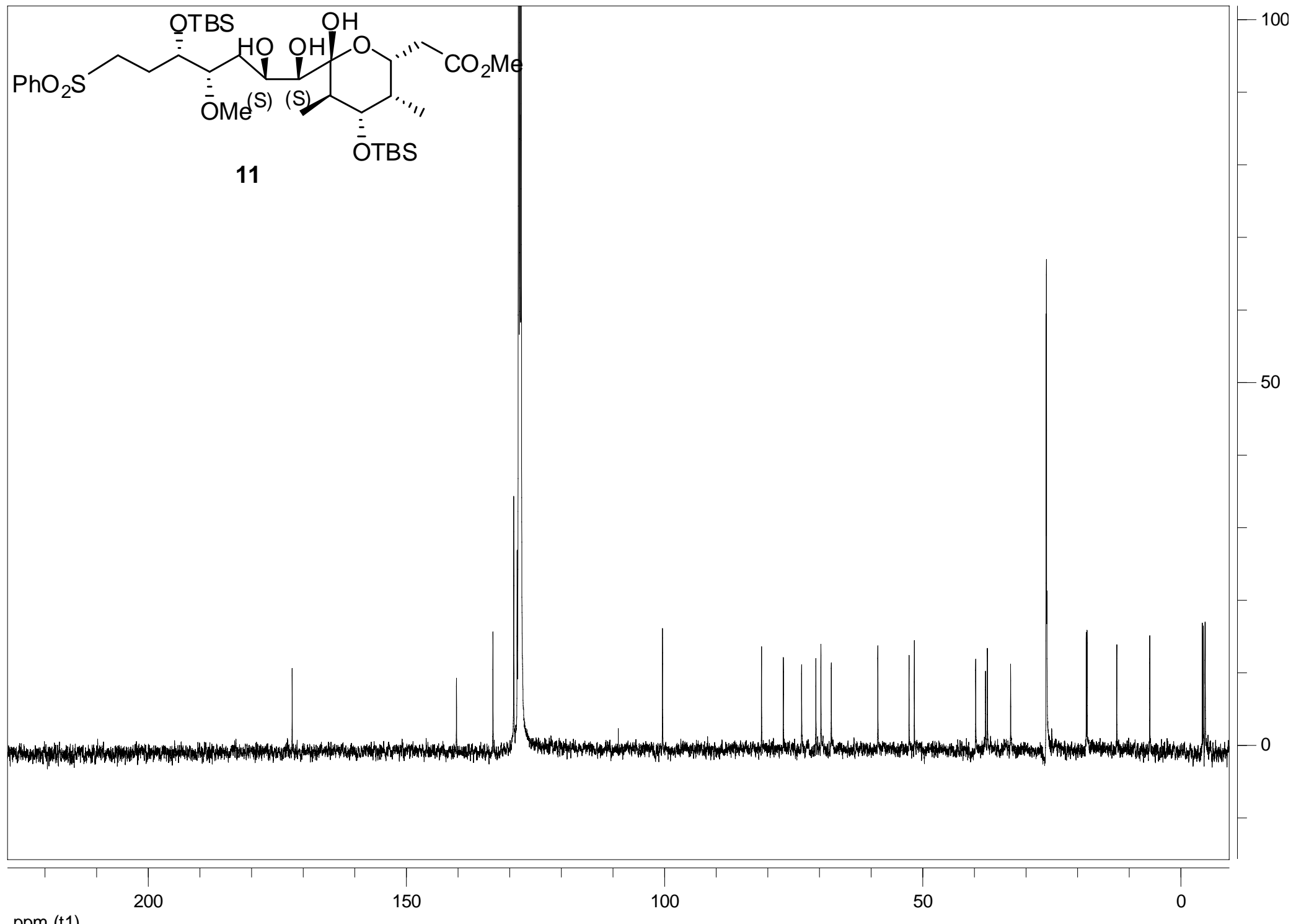

ppm (t1) 


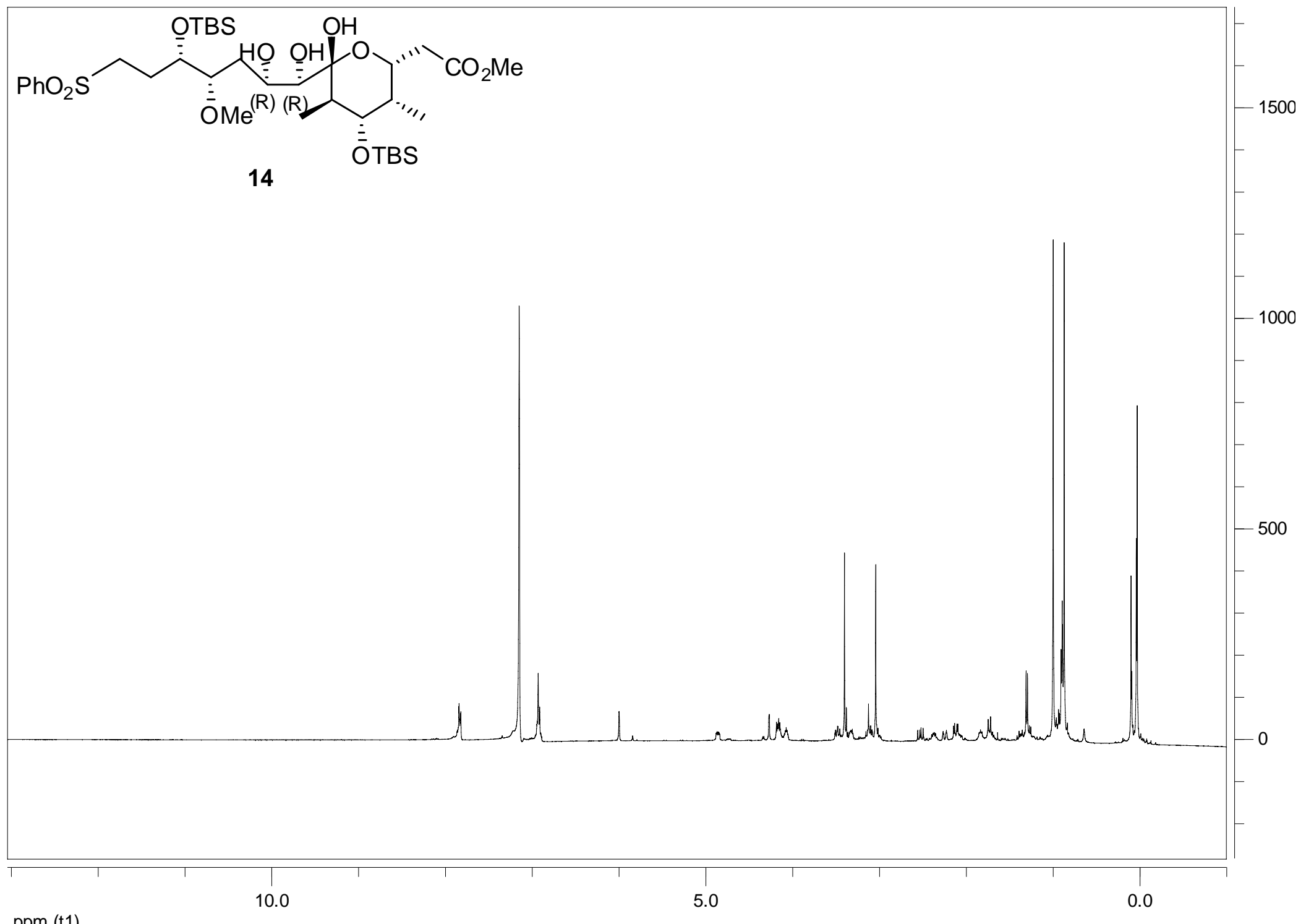

ppm (t1) 


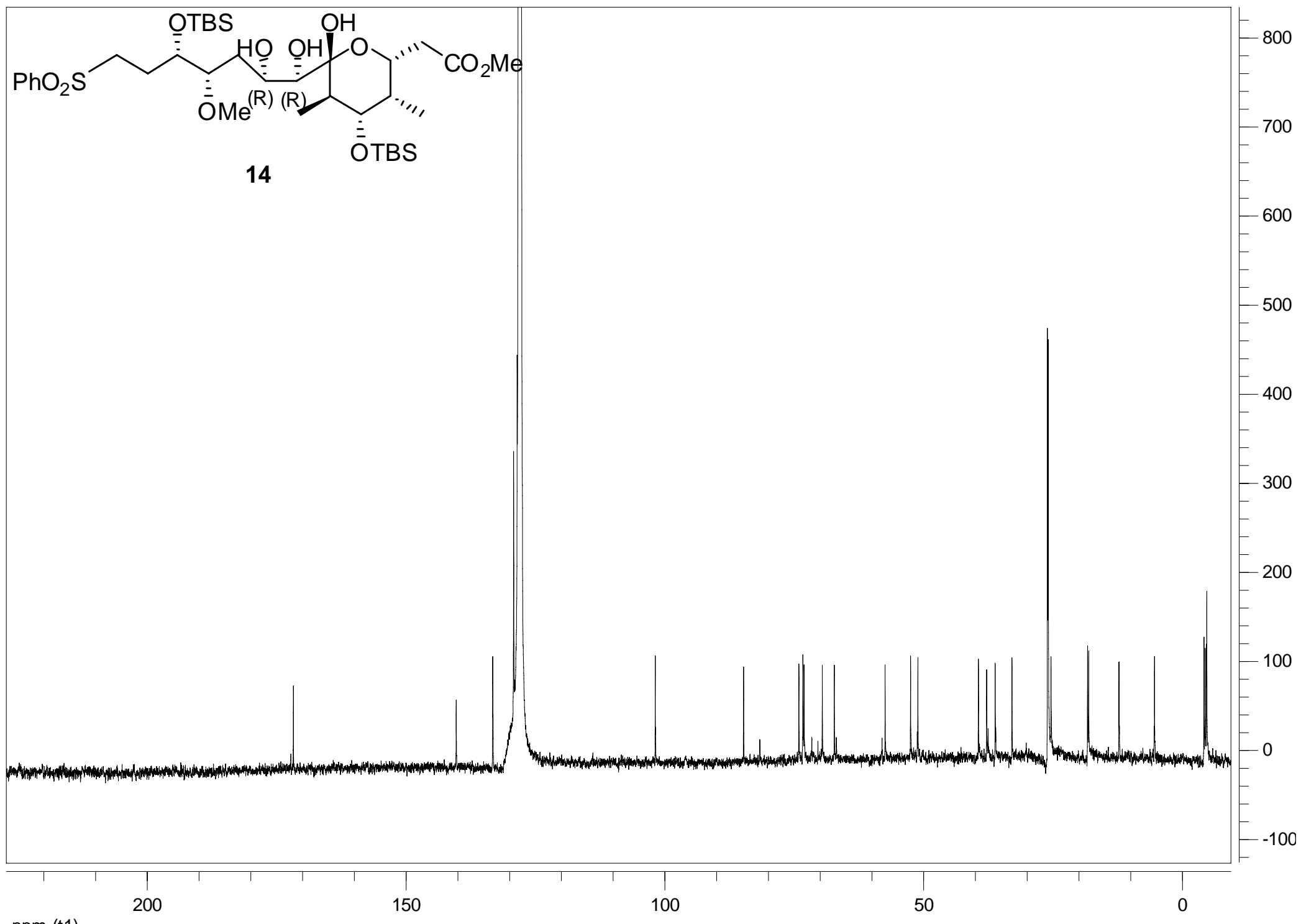

ppm (t1) 


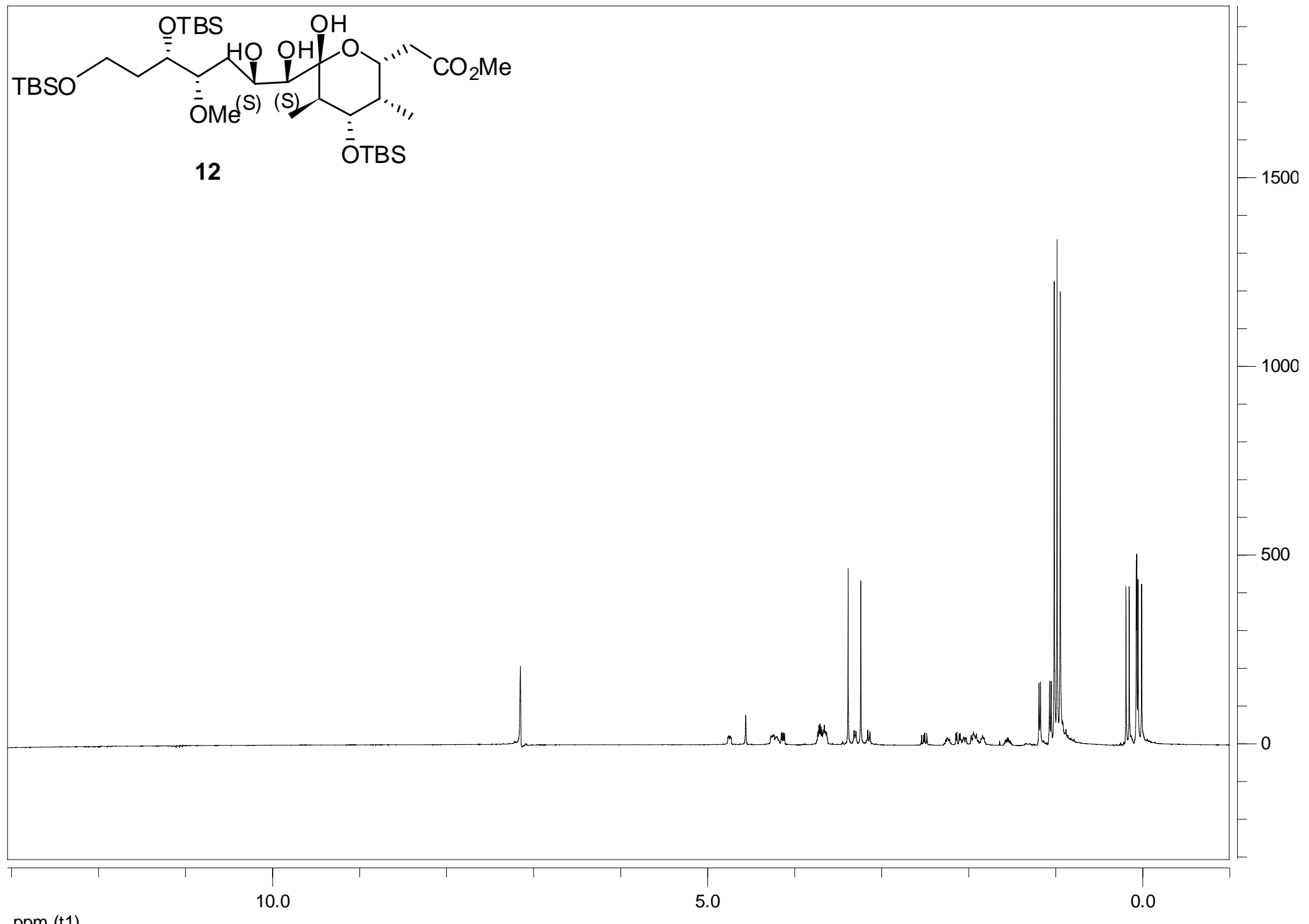

ppm (t1) 


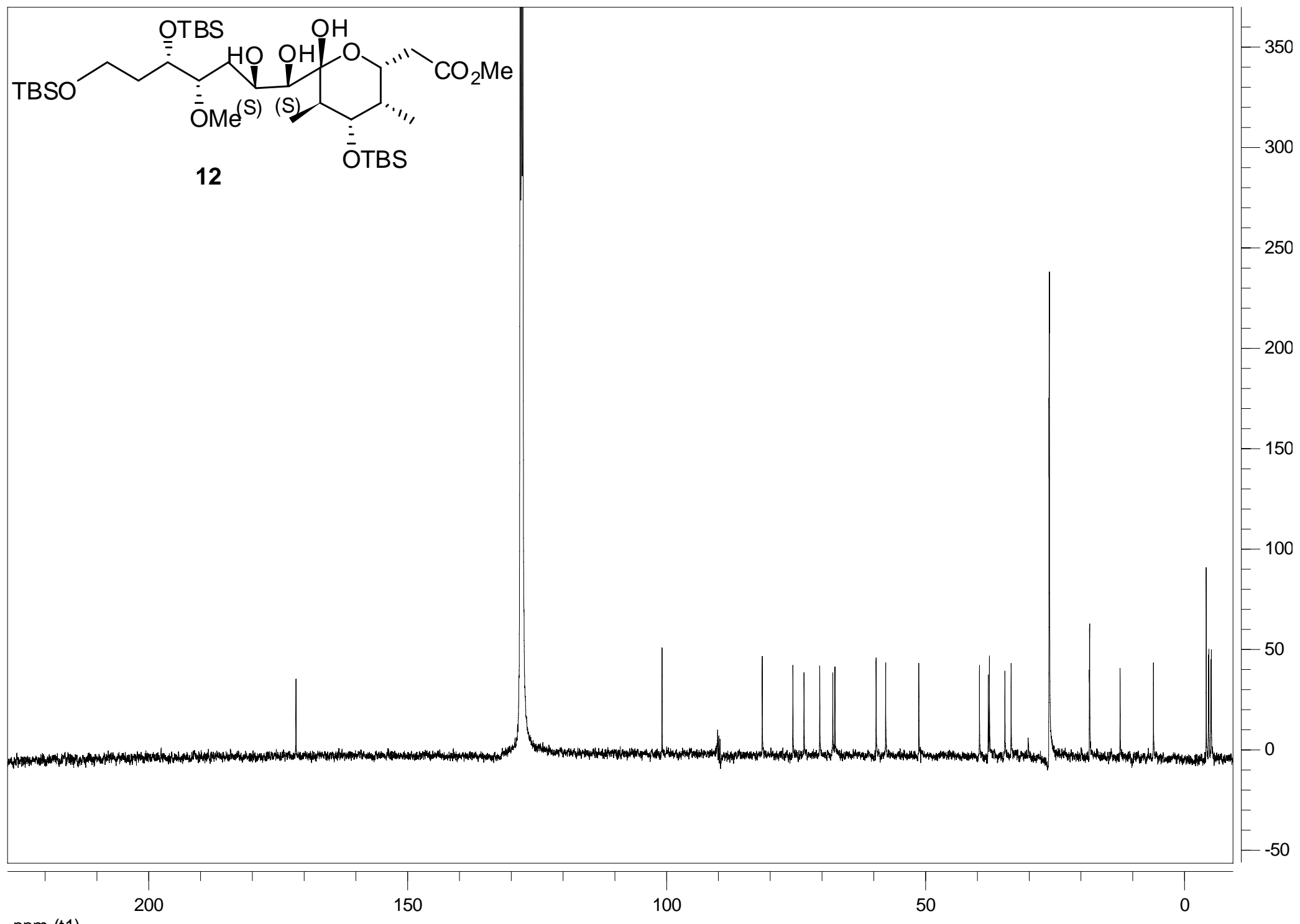

ppm (t1) 


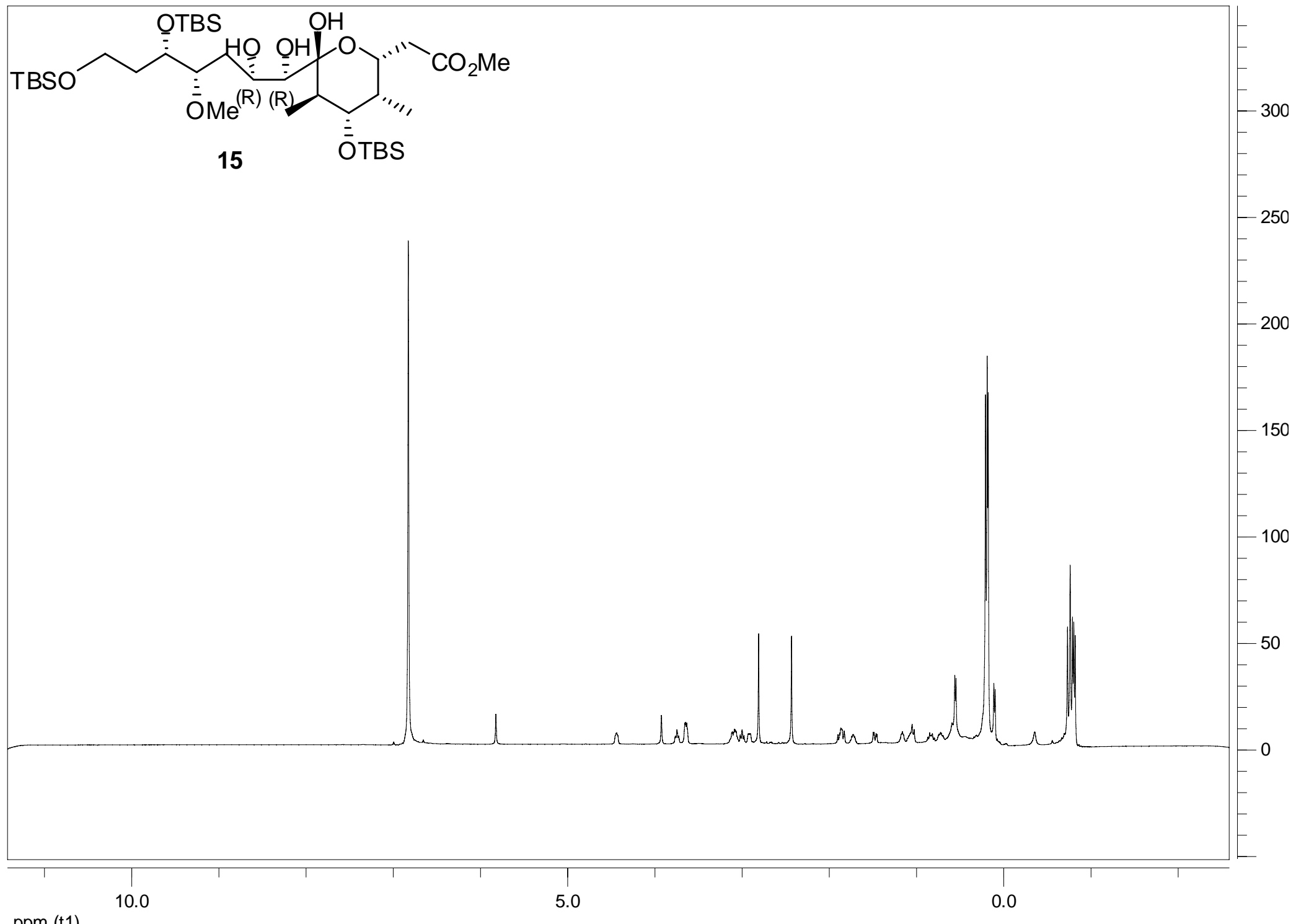

ppm (t1) 


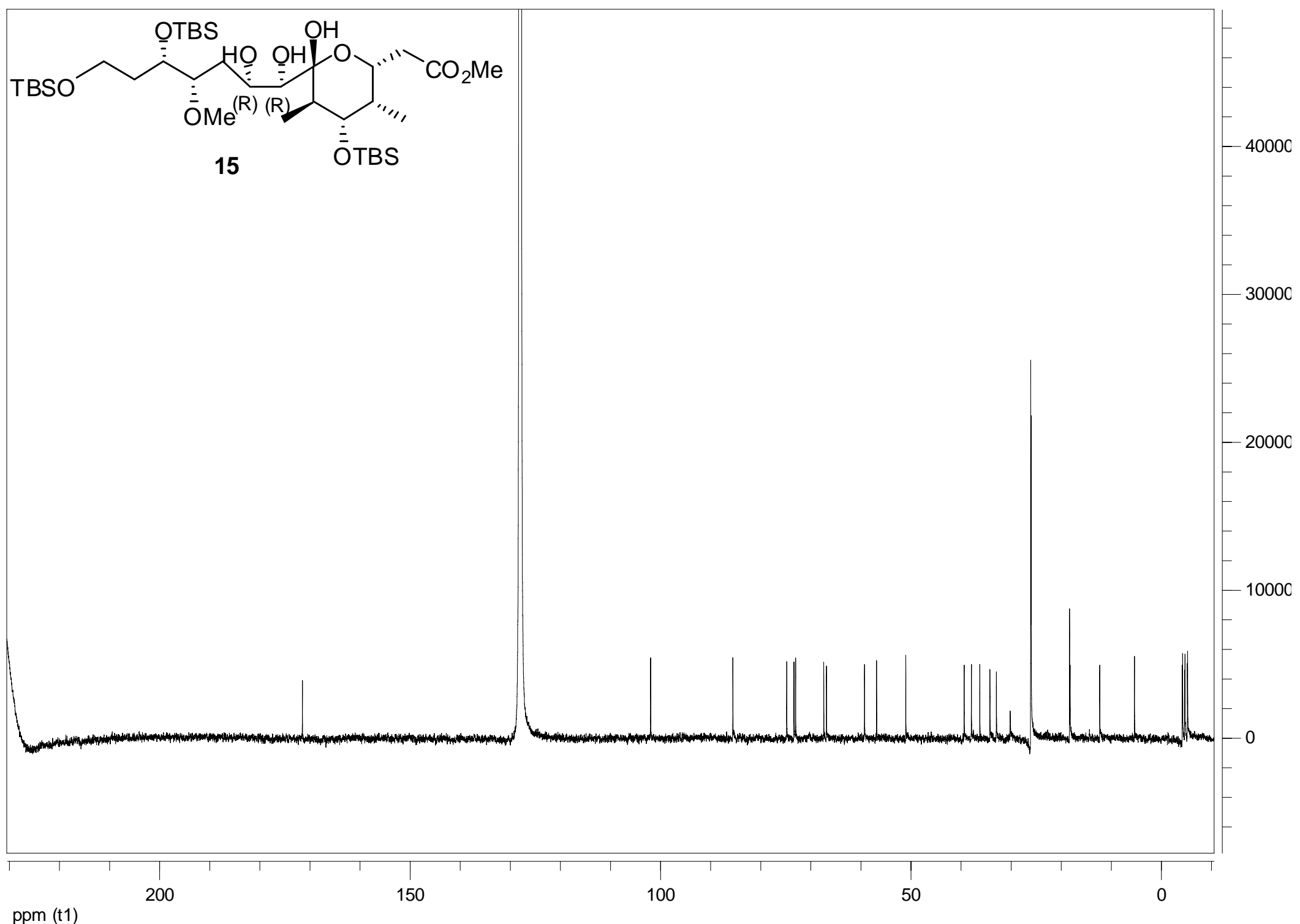




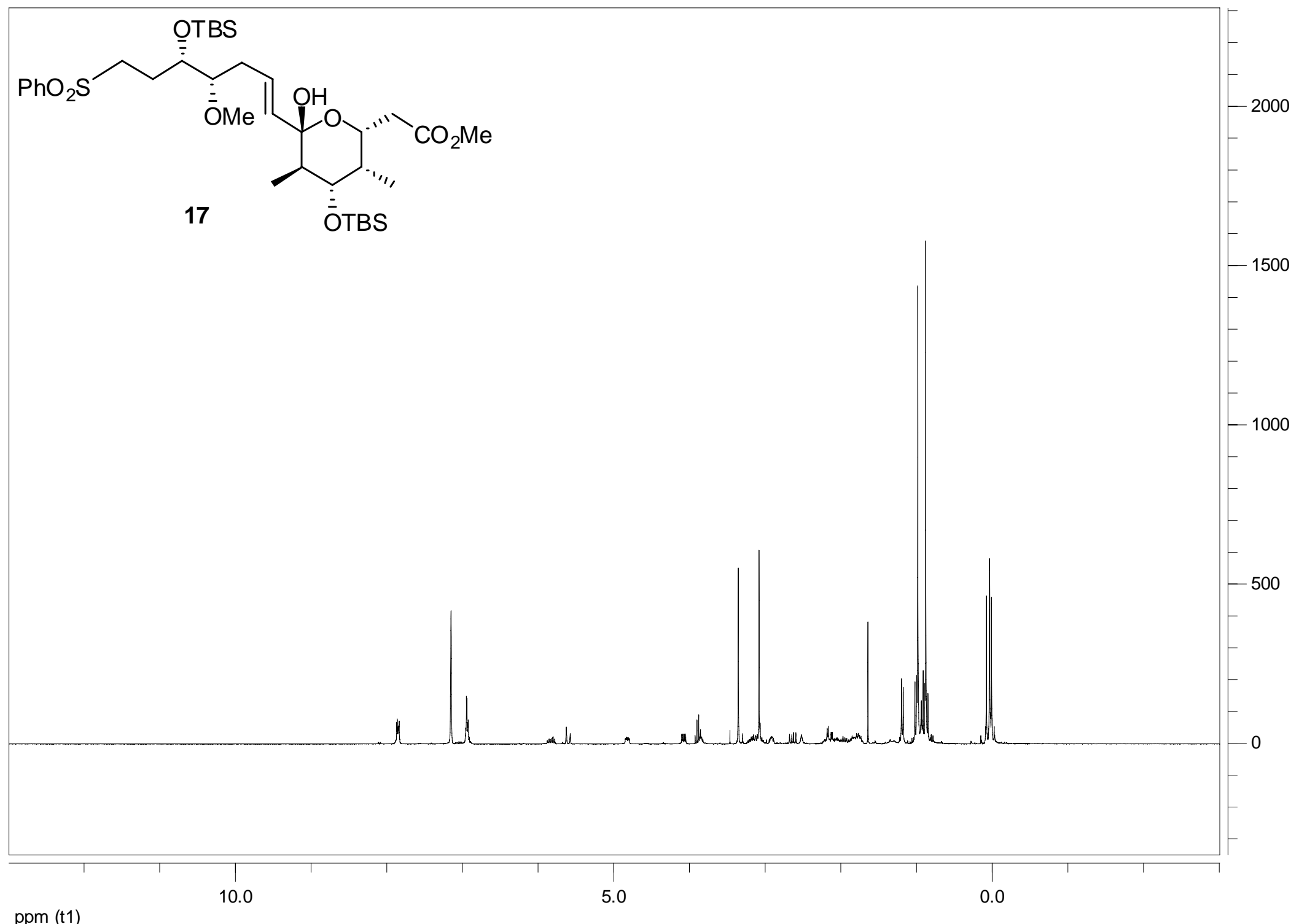




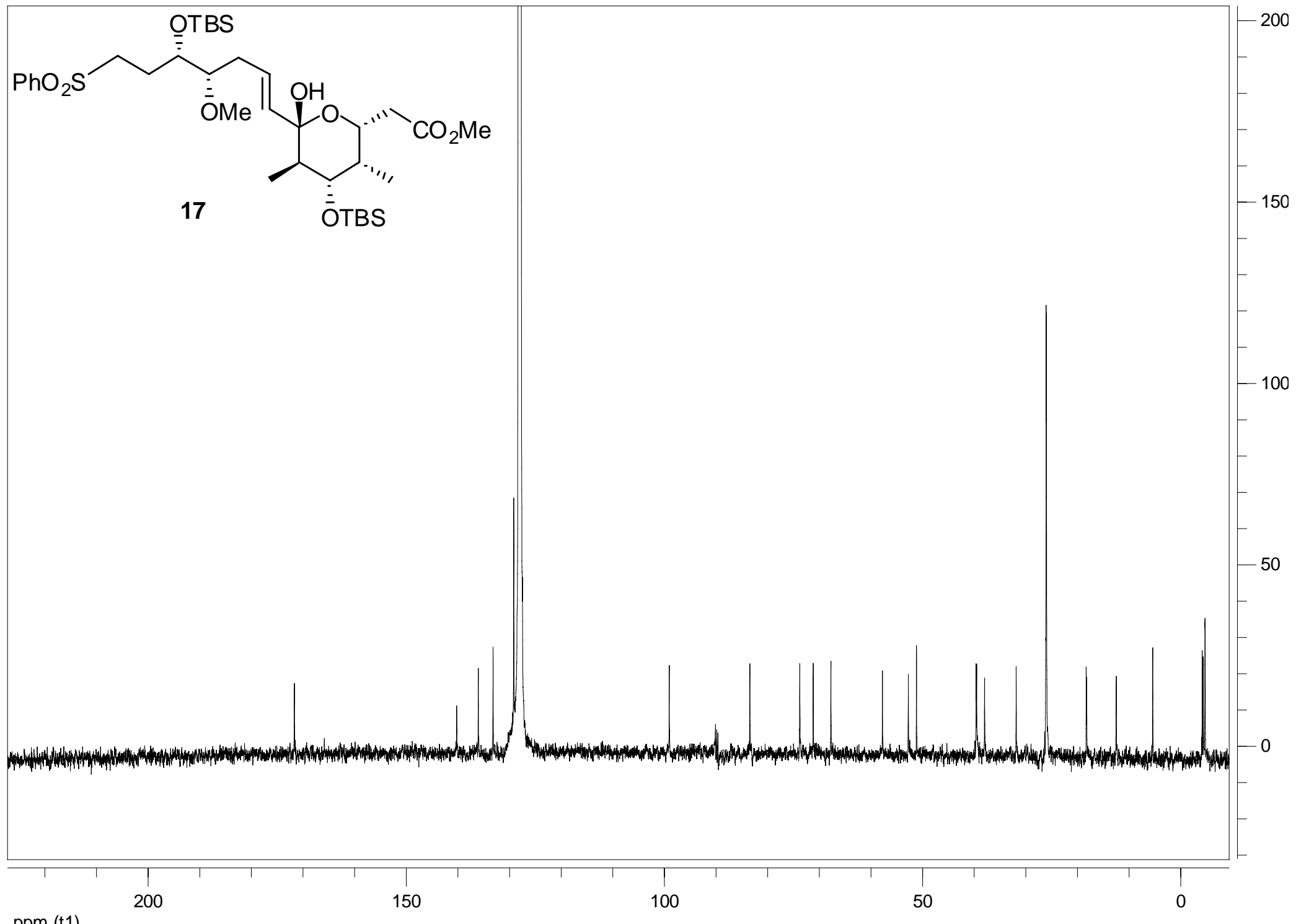

ppm (t1) 


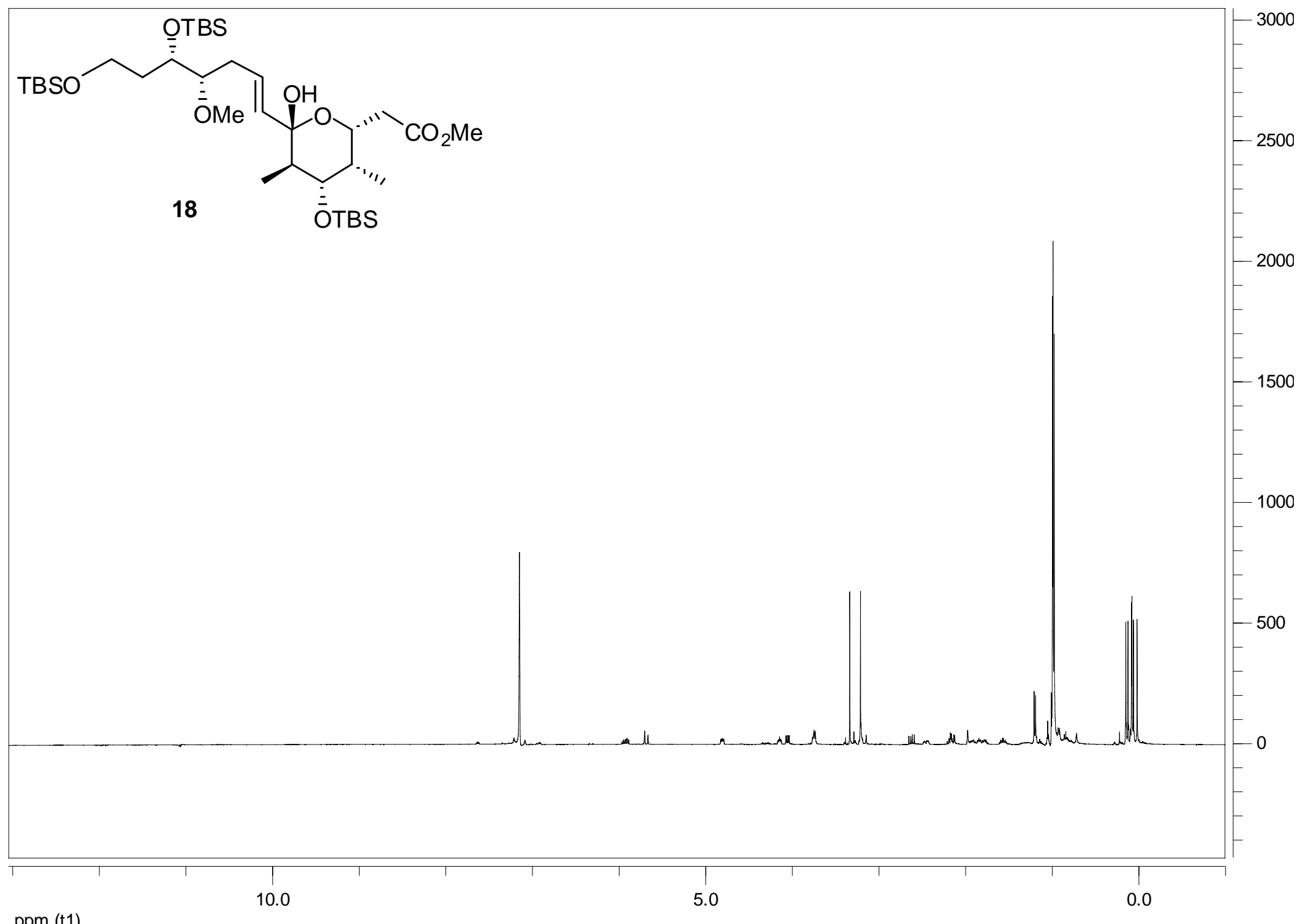

ppm (t1) 


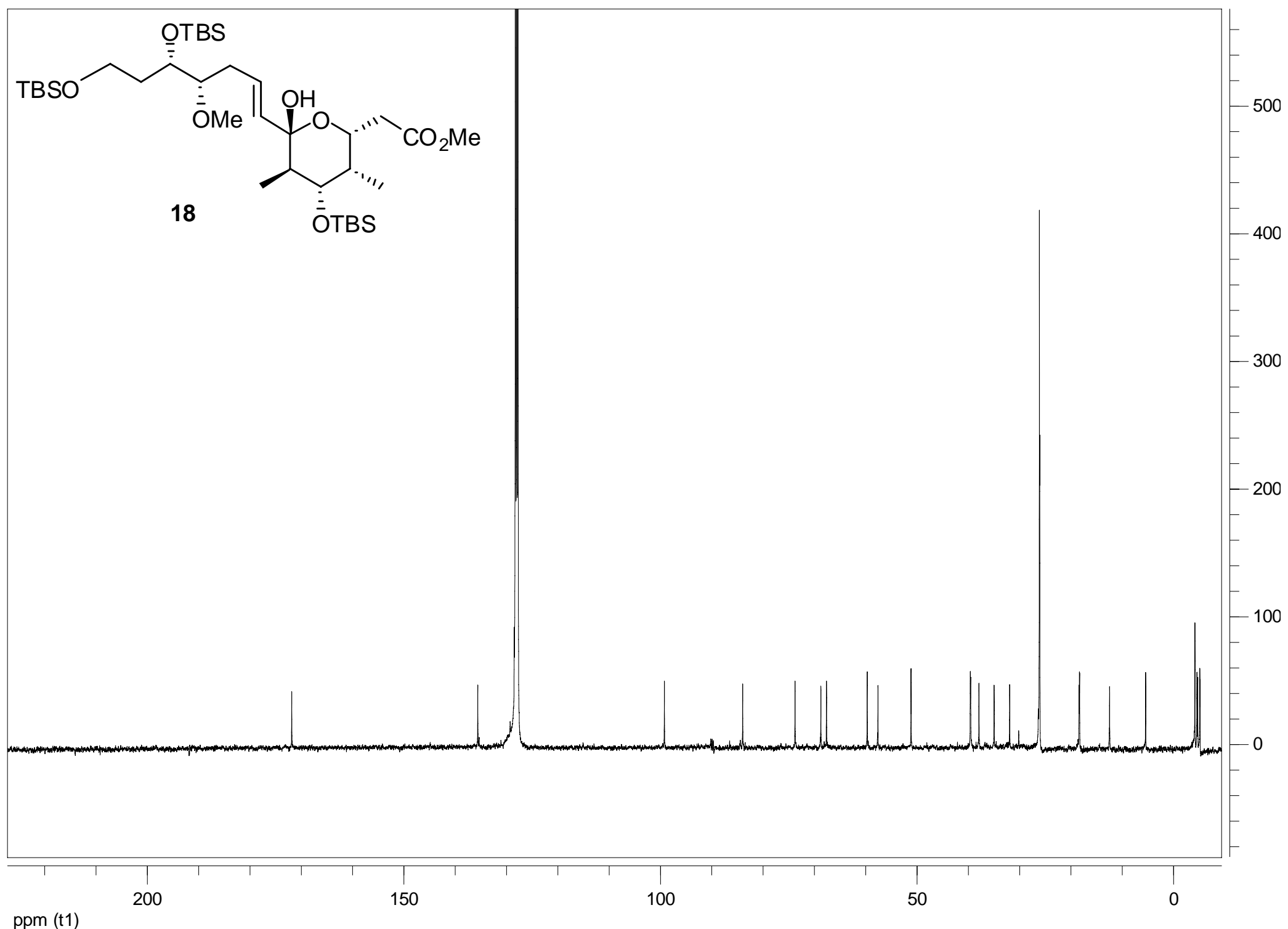




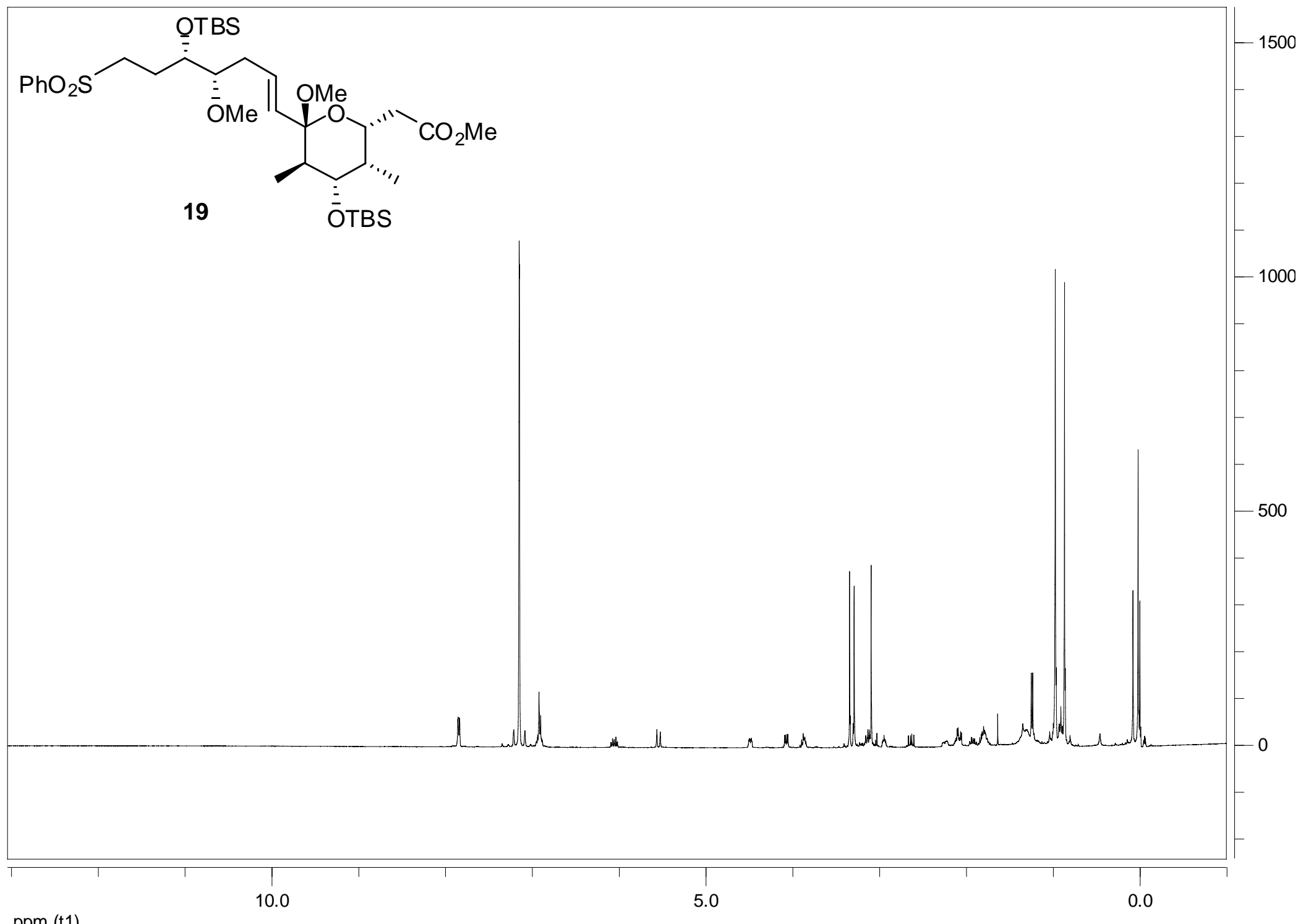

ppm (t1) 


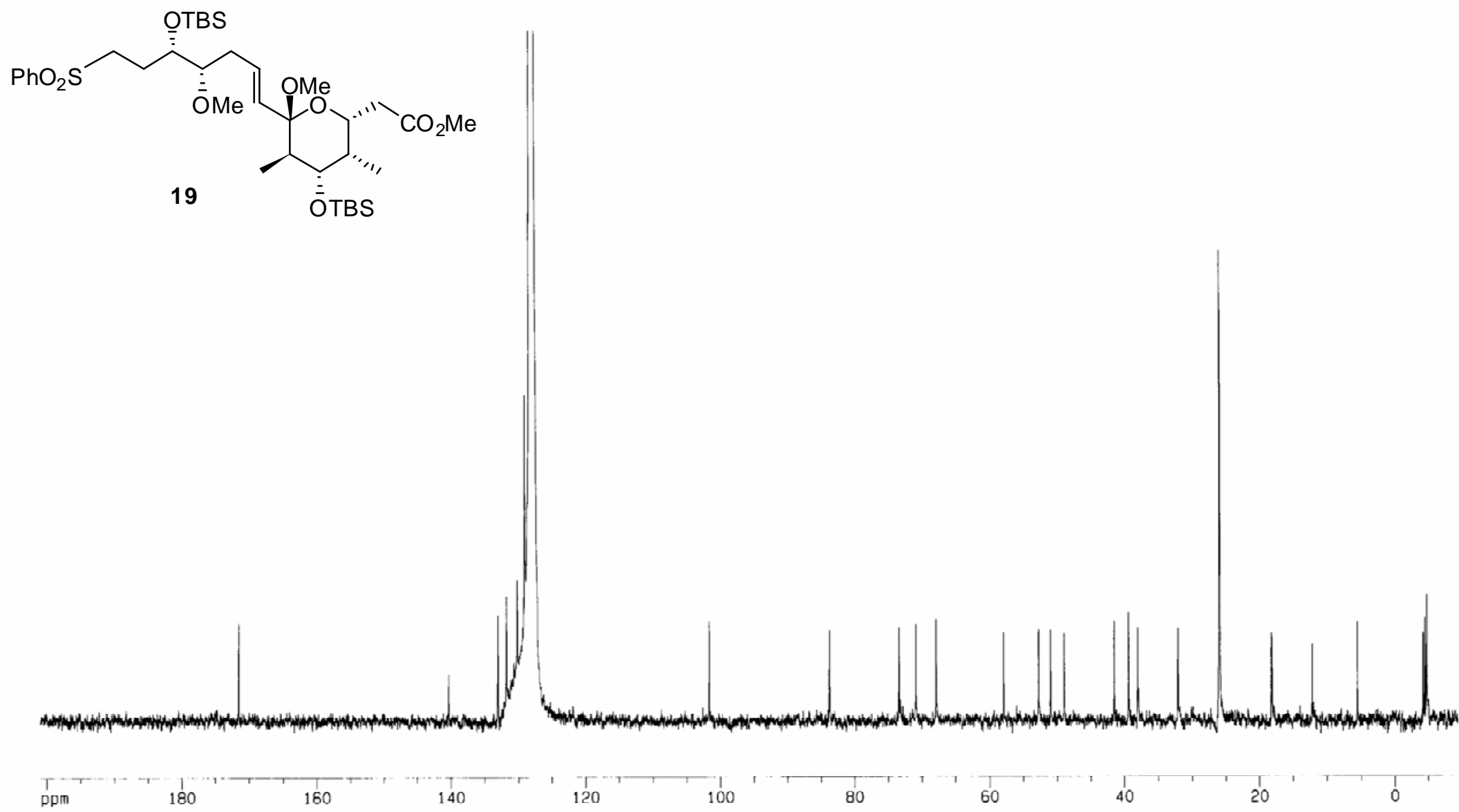




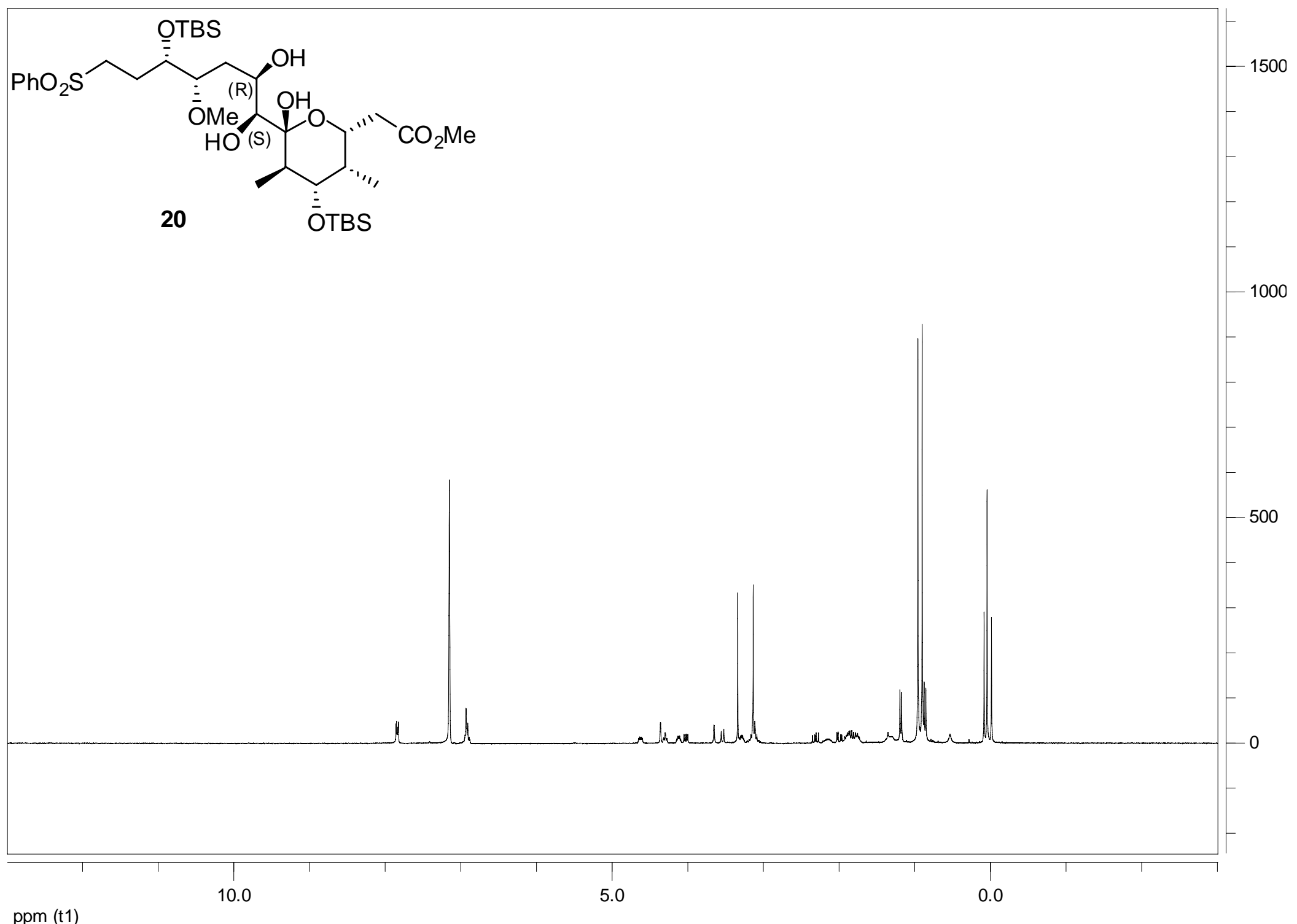




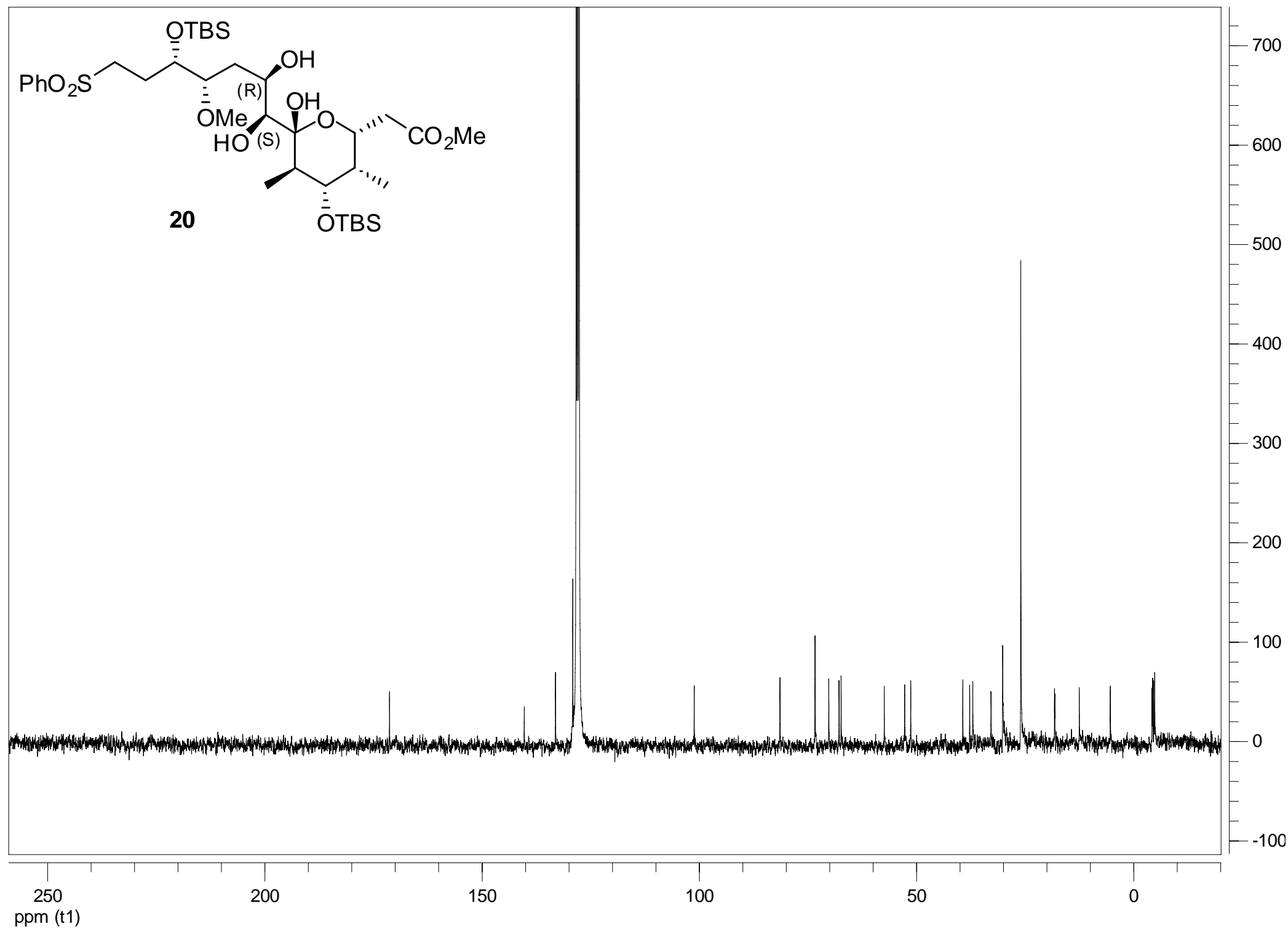




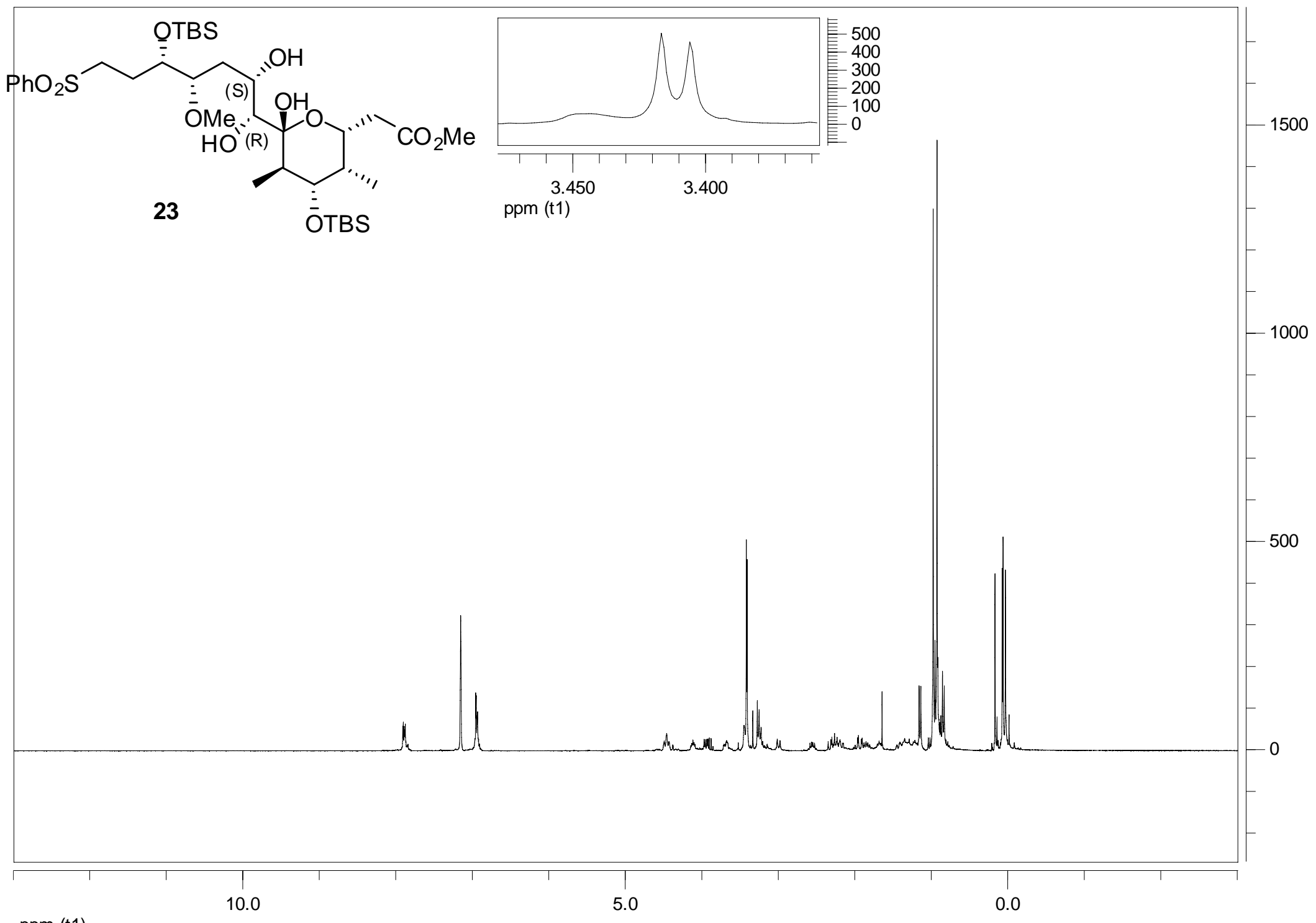

ppm (t1) 


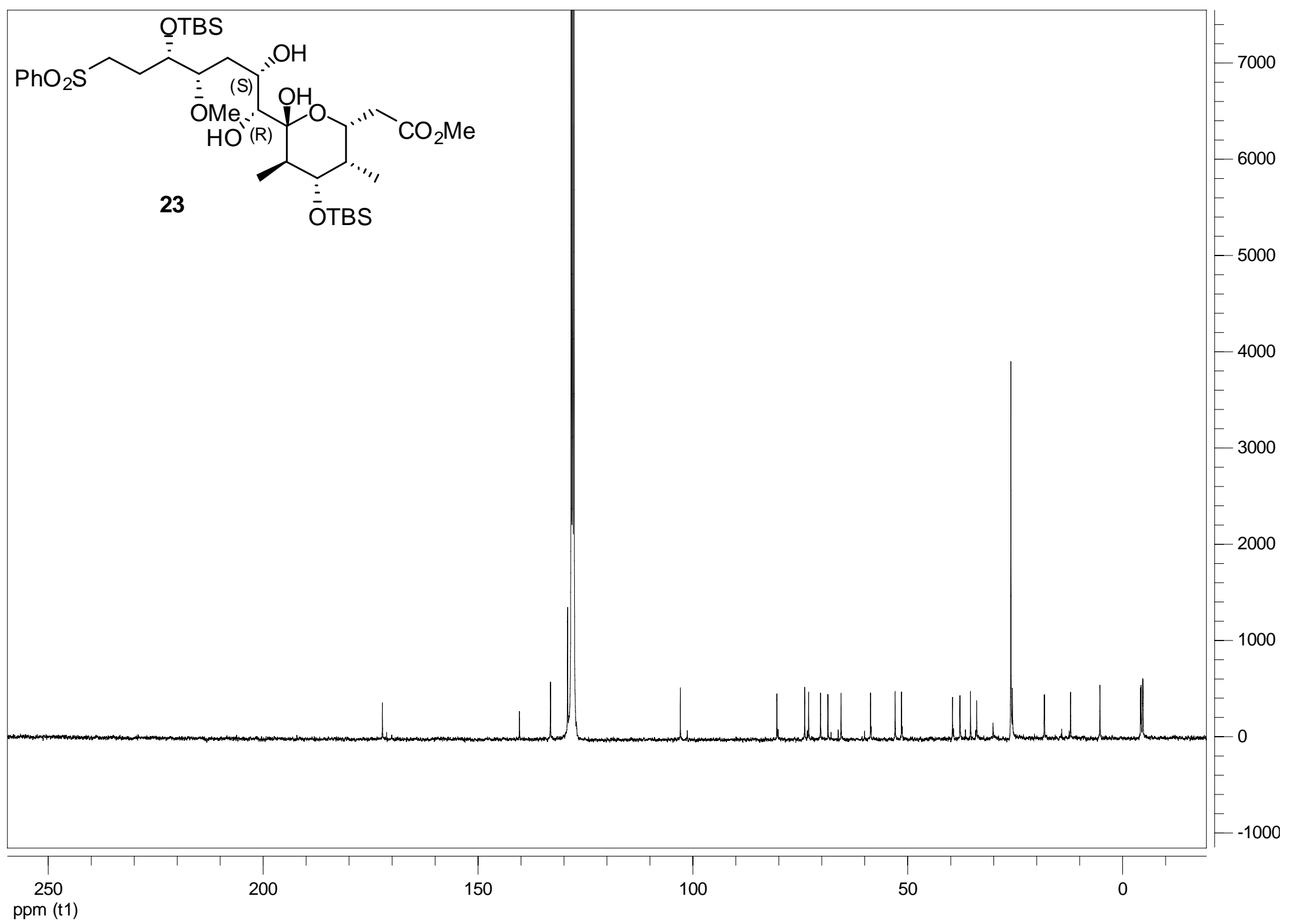




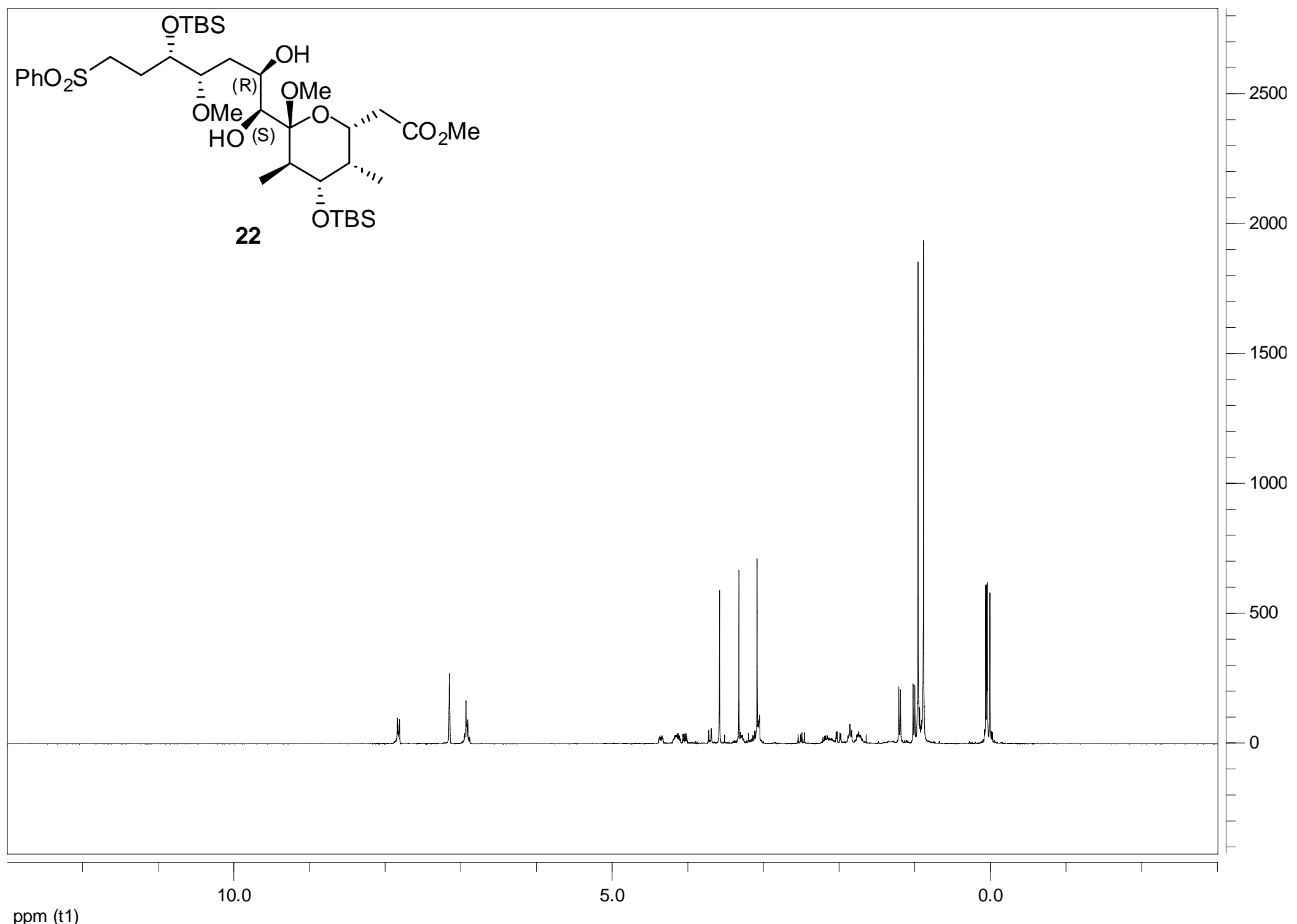




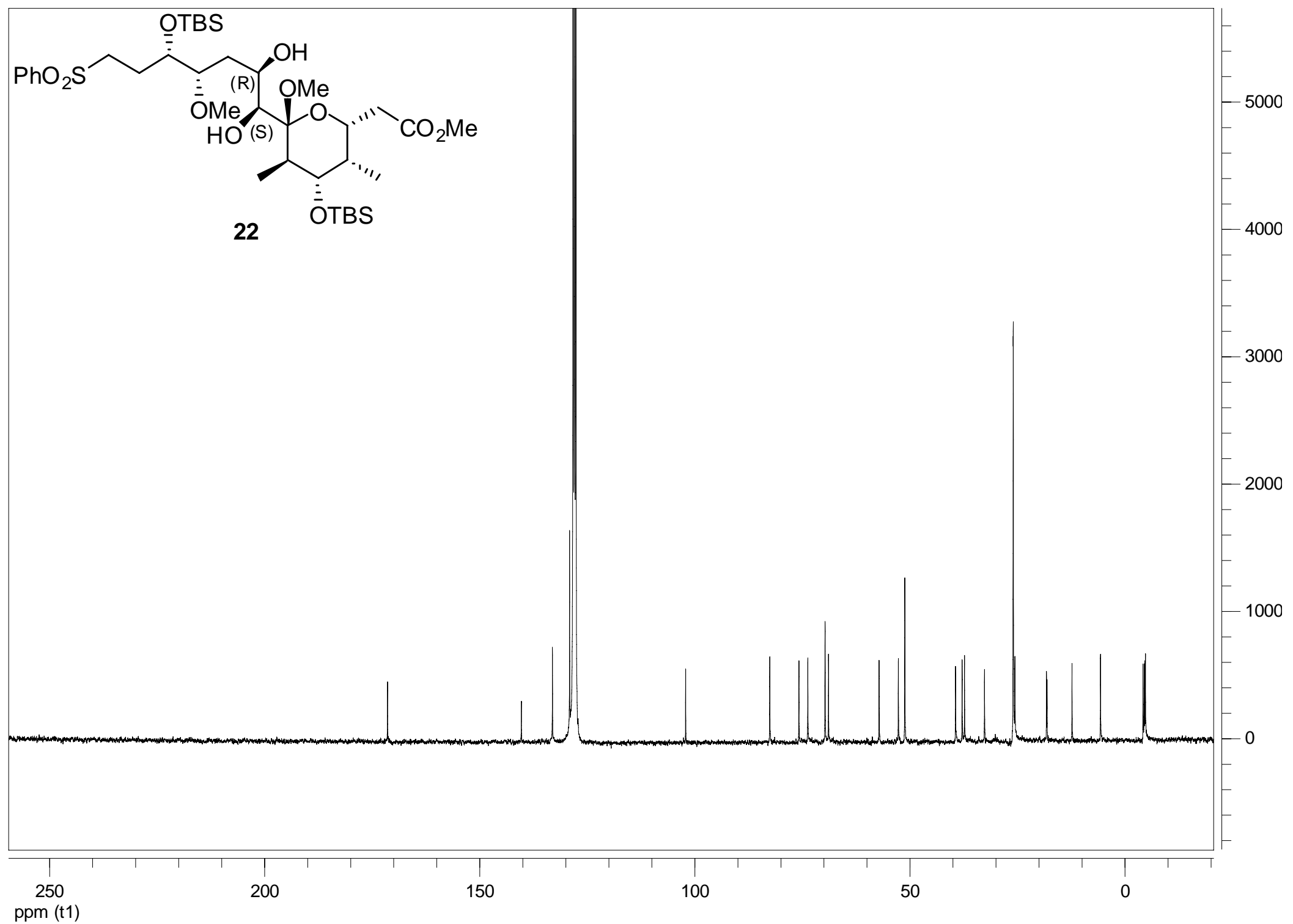




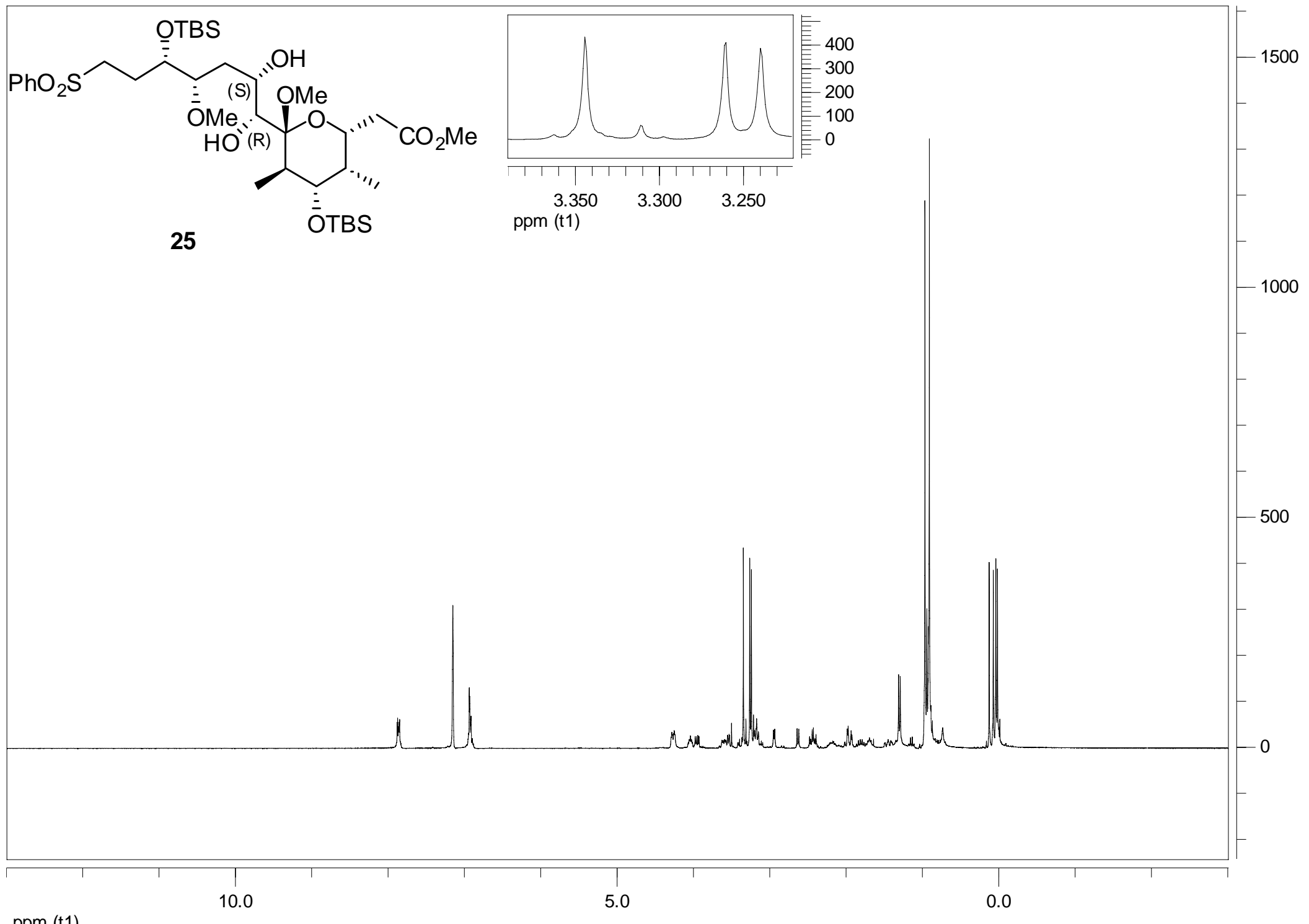

ppm (t1) 


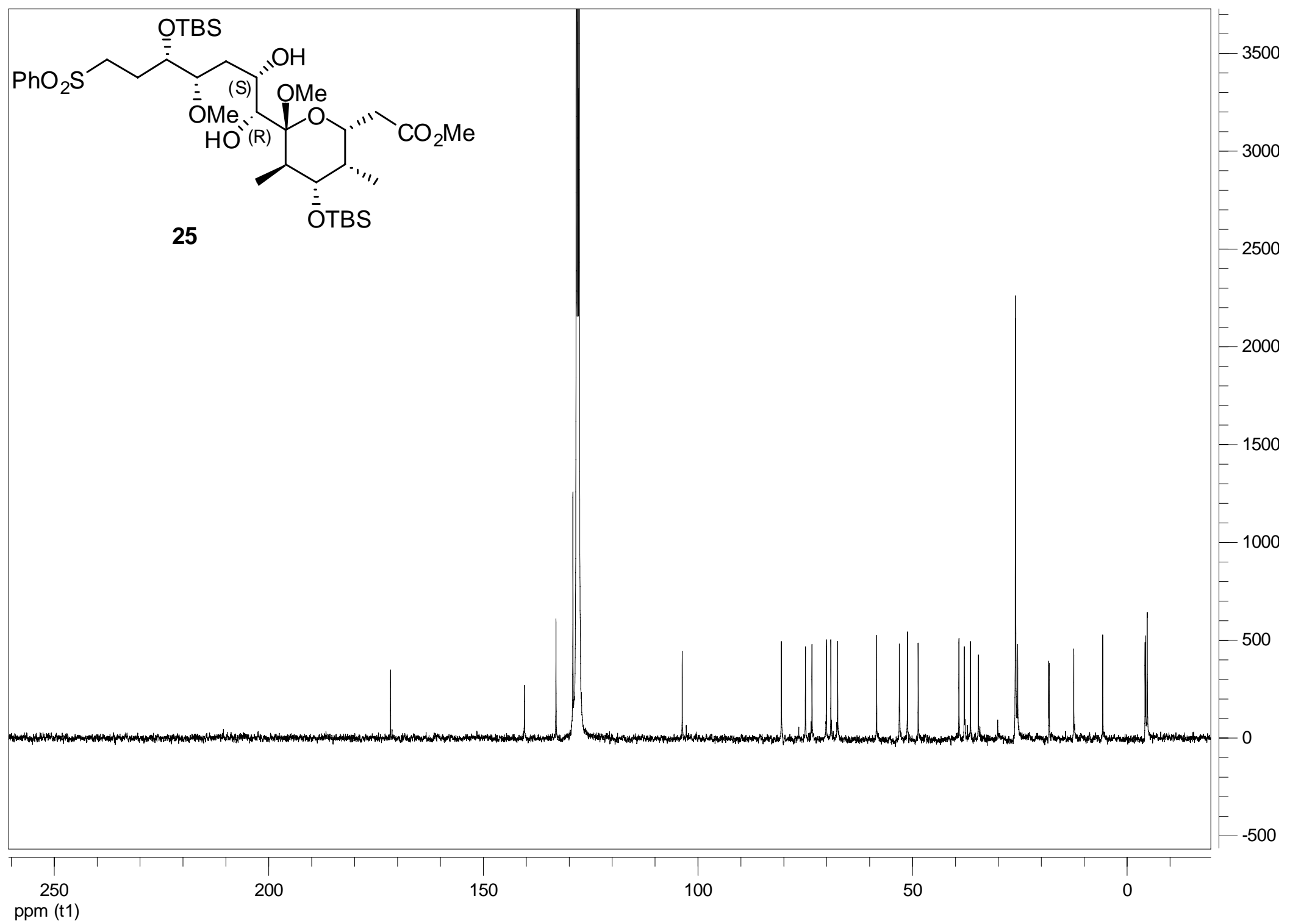




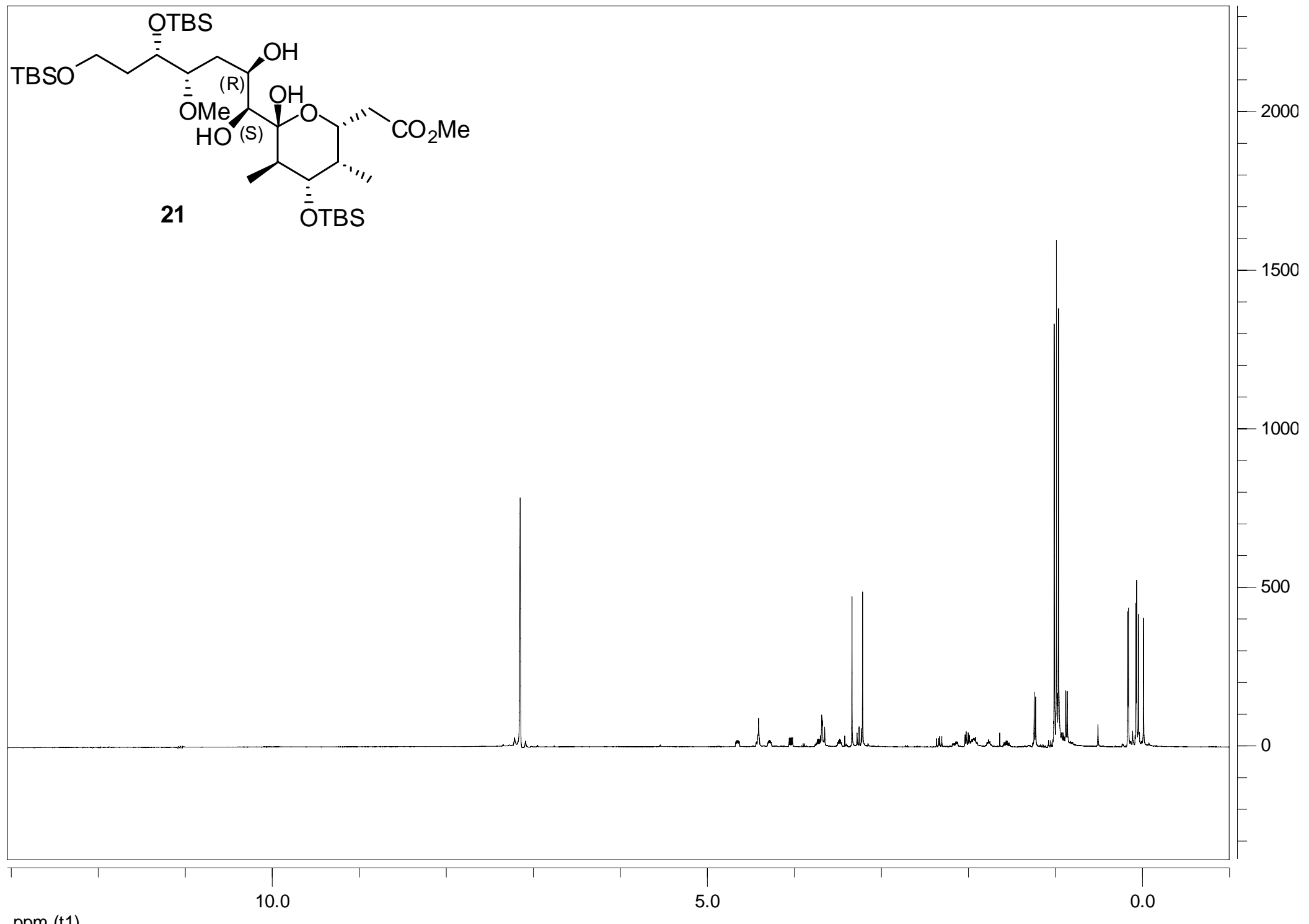

ppm (t1) 


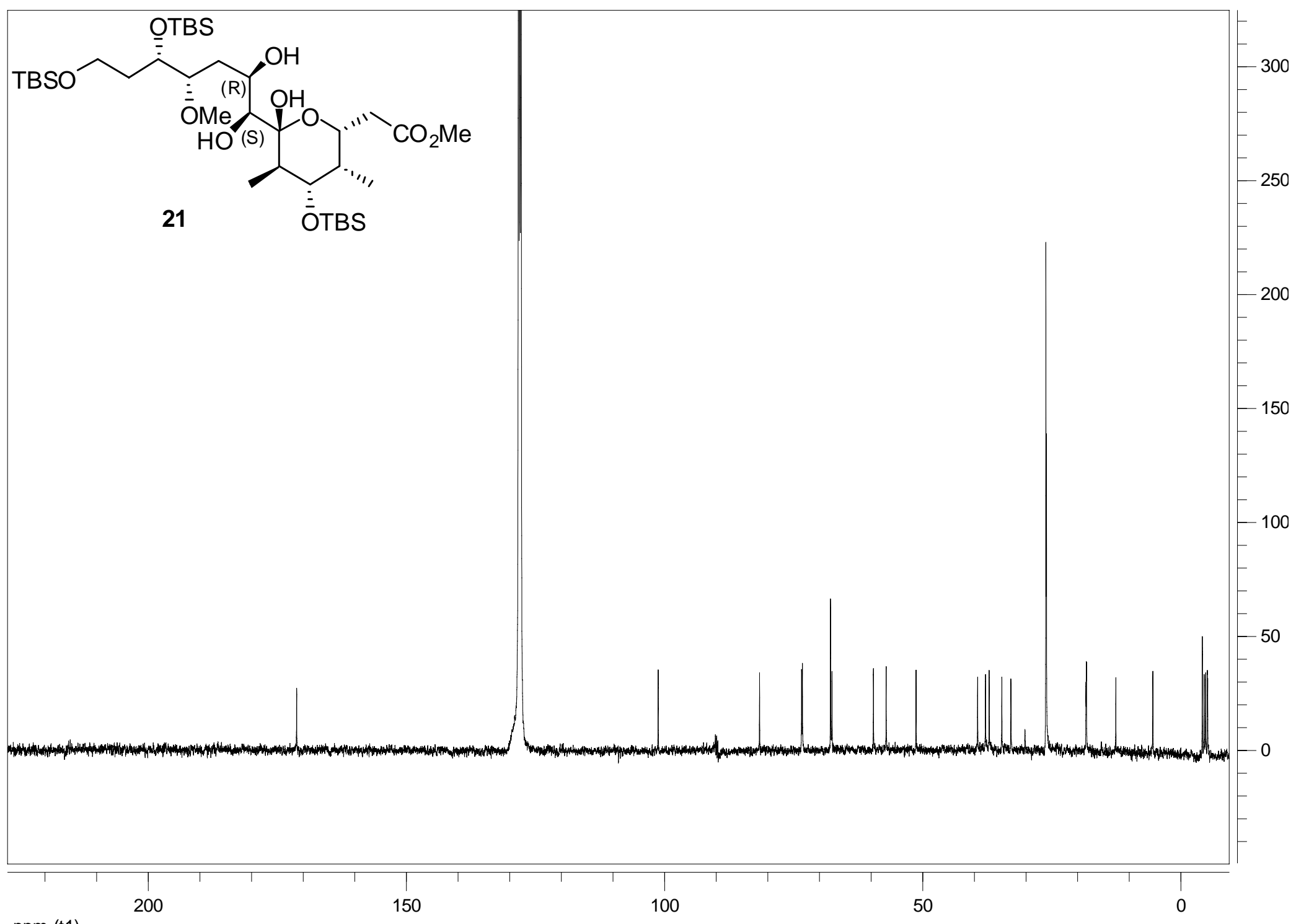

ppm (t1) 


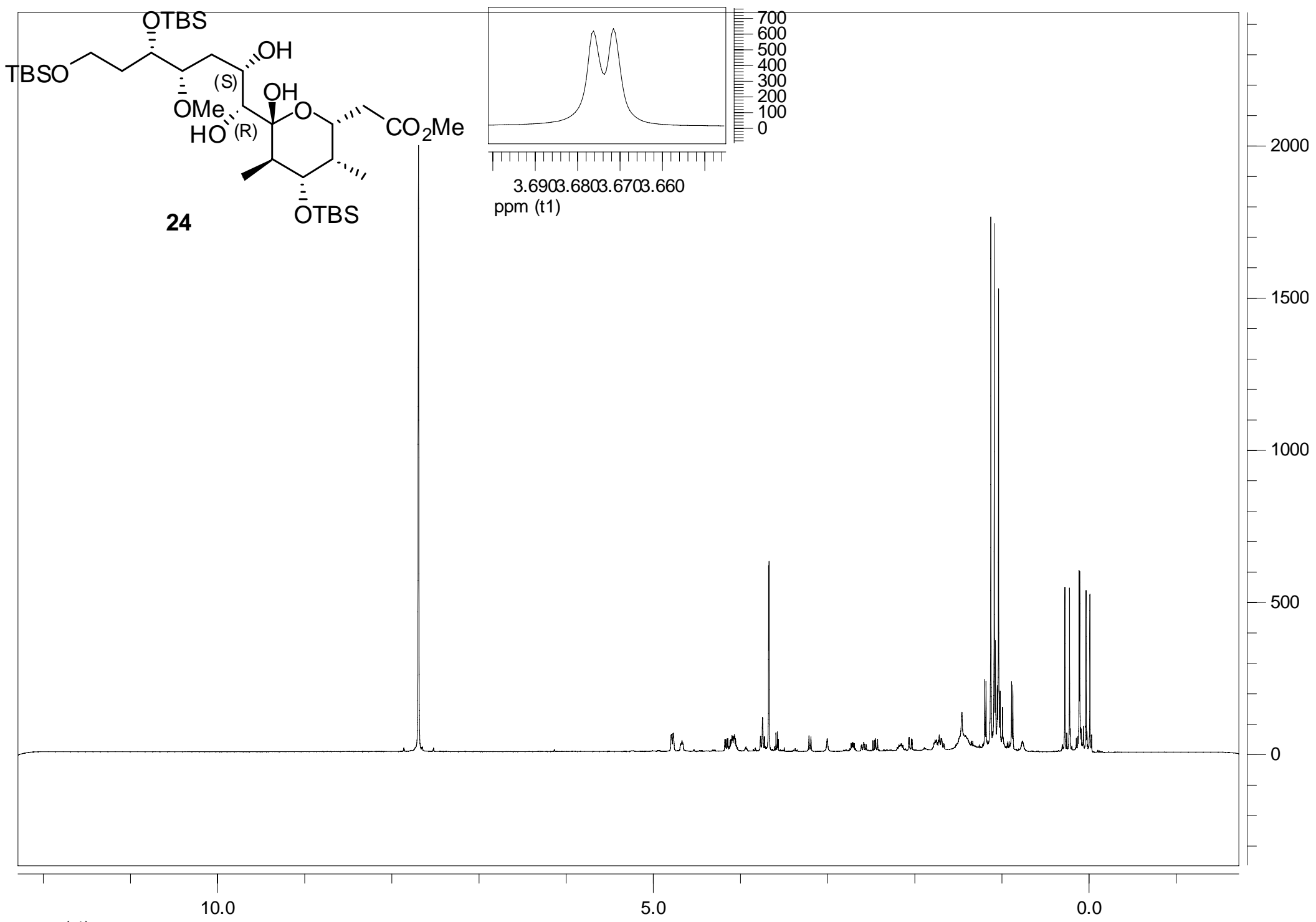

ppm (t1) 


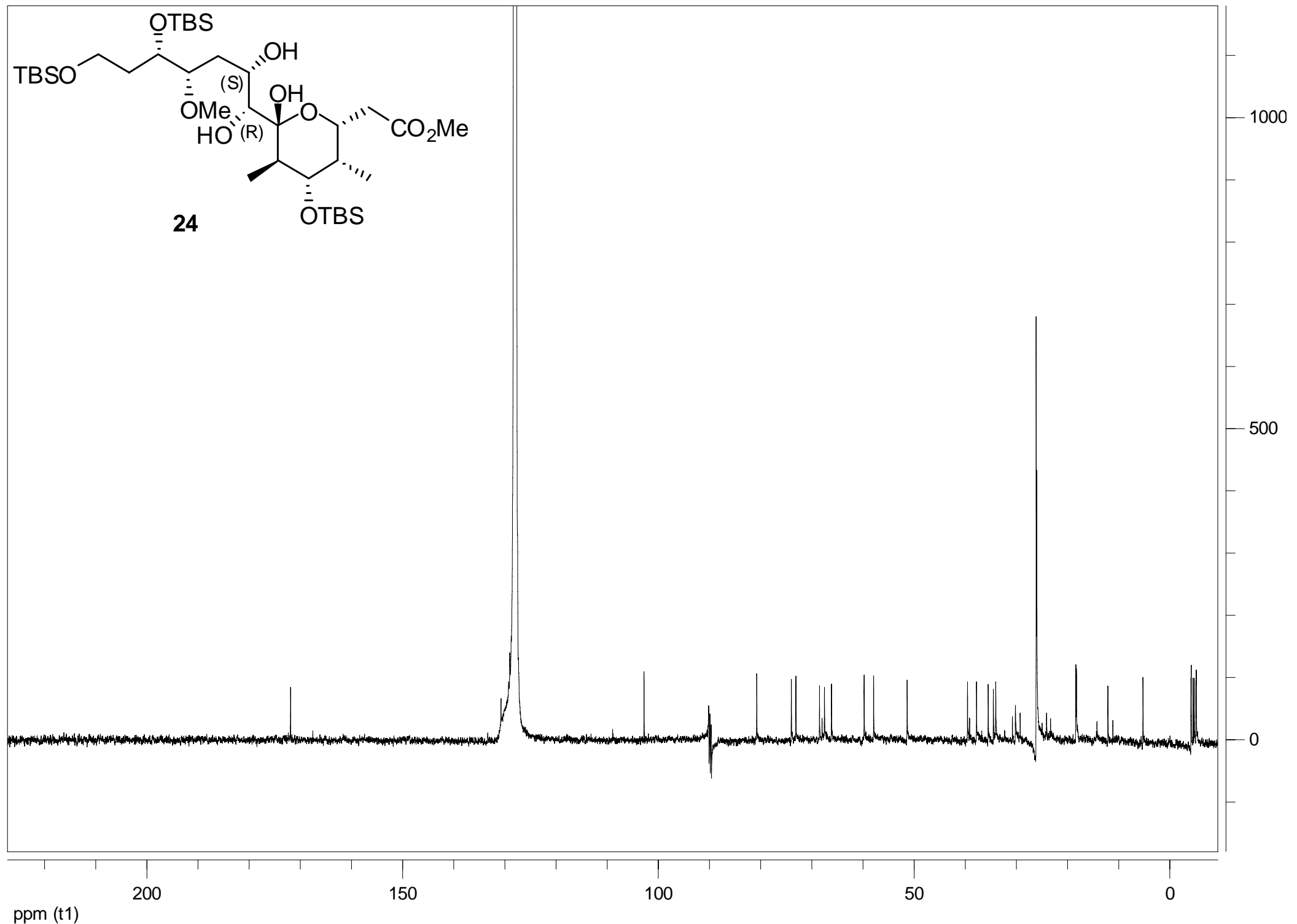




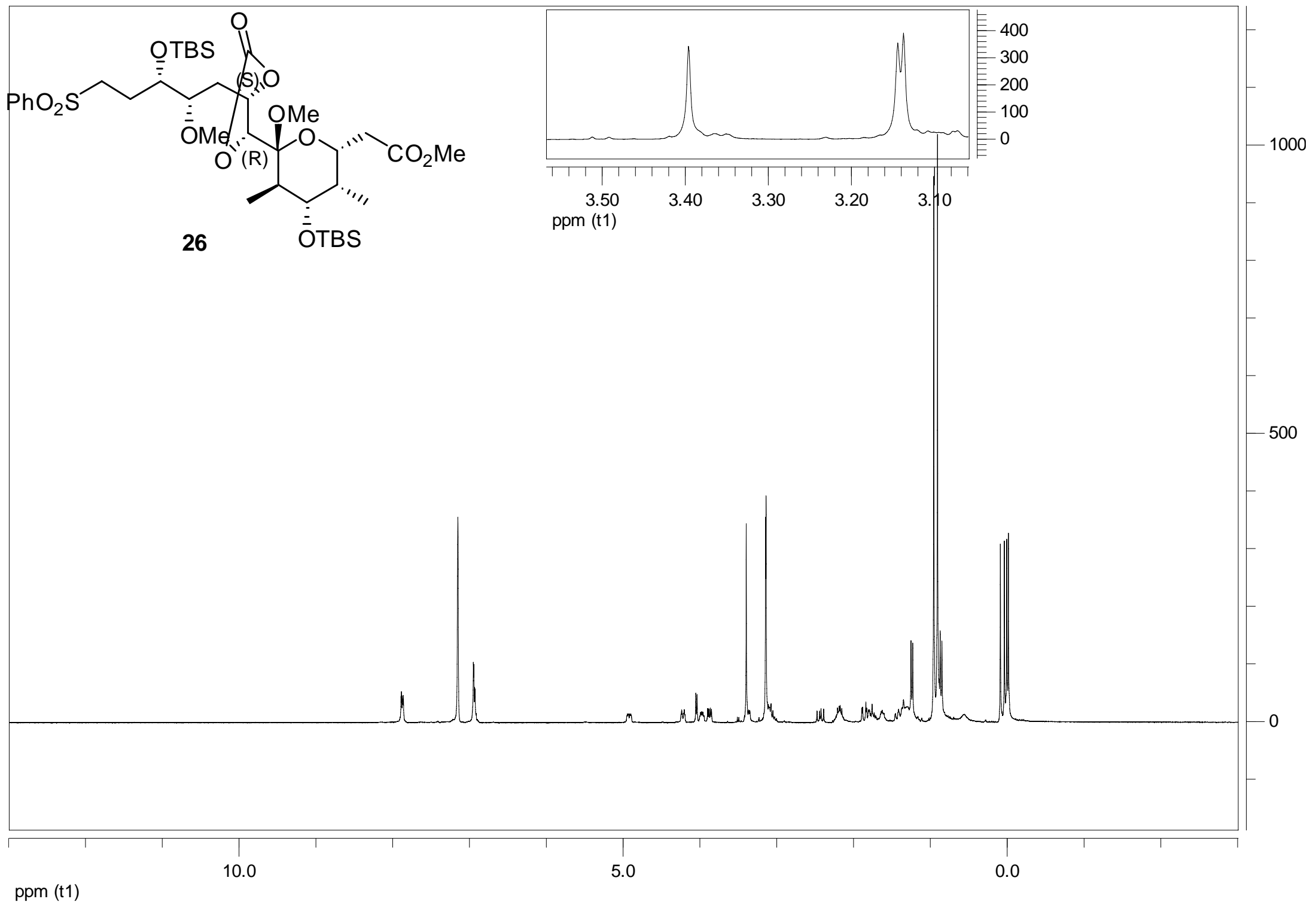




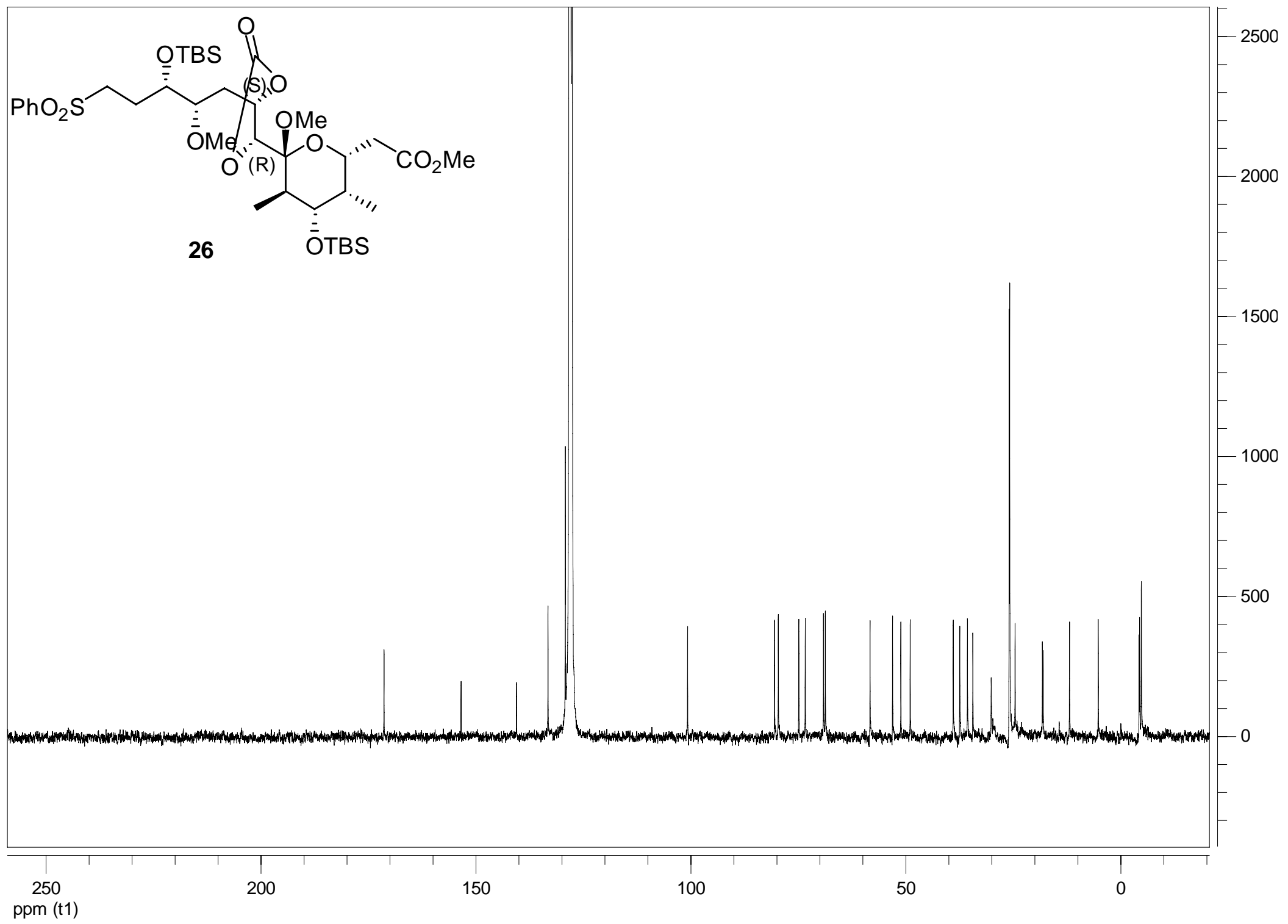




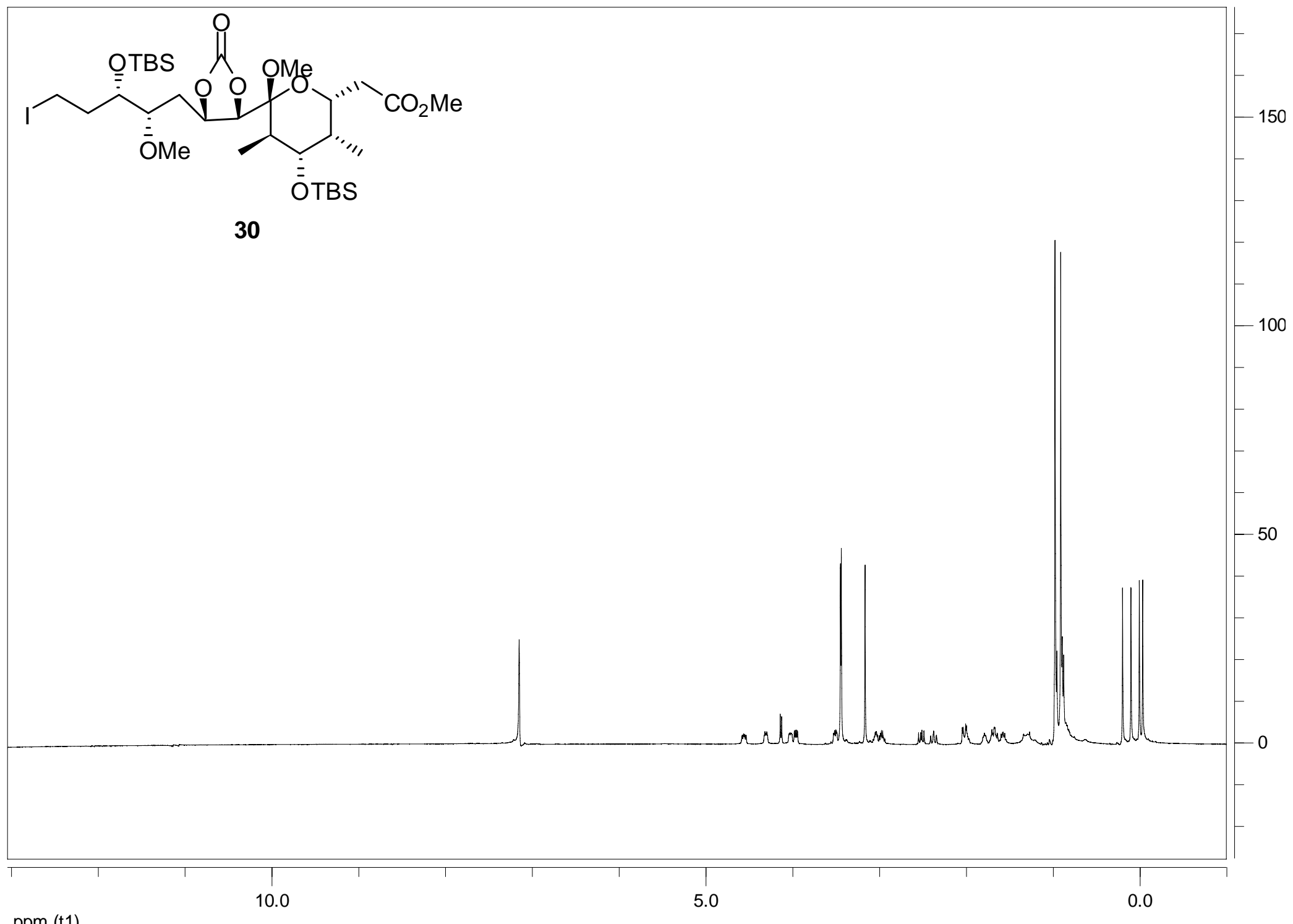

ppm (t1) 


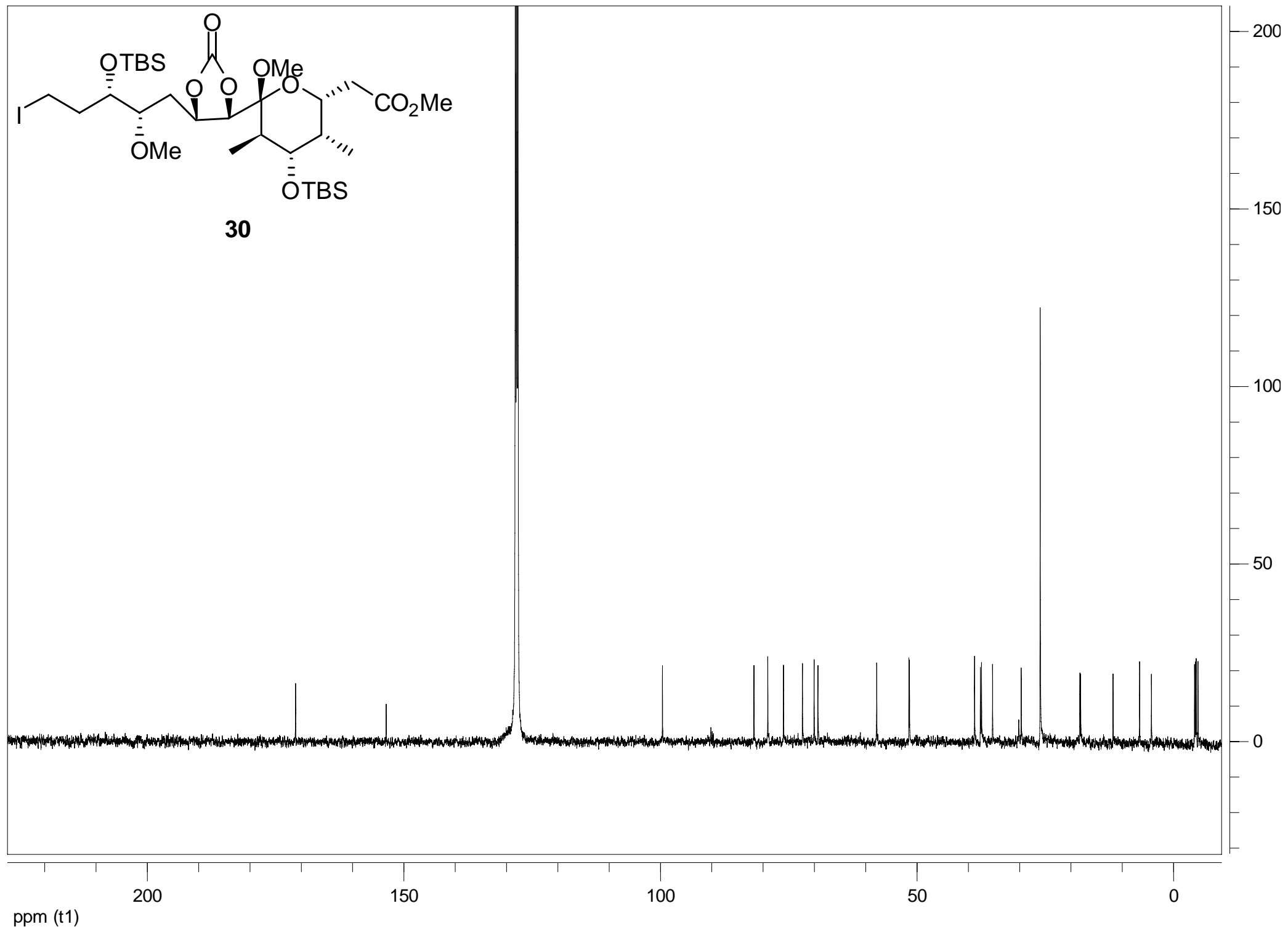

ppm (t1) 